Supporting Information

\title{
Structure-based exploration of selectivity for ATM inhibitors in Huntington's disease
}

Amanda Van de Poël, ${ }^{\wedge}$ Leticia Toledo-Sherman, ${ }^{\wedge}+$ Perla Breccia, ${ }^{\wedge}{ }^{\dagger}$ Roger Cachope, $\stackrel{\star}{ }$ Jennifer R. Bate, ${ }^{\dagger}$ Ivan Angulo-Herrera, ${ }^{\dagger}$ Grant Wishart,${ }^{\dagger}$ Kim L. Matthews,${ }^{\dagger}$ Sarah L. Martin, ${ }^{\dagger}$ Marcus Peacock, ${ }^{\dagger}$ Amy Barnard,${ }^{\dagger}$ Helen C. Cox,${ }^{\dagger}$ Graham Jones,${ }^{\dagger}$ George McAllister, ${ }^{\dagger}$ Huw Vater, ${ }^{\dagger}$ William Esmieu, ${ }^{\dagger}$ Cole Clissold,${ }^{\dagger}$ Marieke Lamers, ${ }^{\dagger}$ Philip Leonard,${ }^{\dagger}$ Rebecca E. Jarvis, ${ }^{\dagger}$ Wesley Blackaby, ${ }^{\dagger}$ Maria Eznarriaga, ${ }^{\dagger}$ Ovadia Lazari, ${ }^{\dagger}$ Dawn Yates,${ }^{\dagger}$ Mark Rose,,${ }^{\dagger}$ Sung-Wook Jang, ${ }^{\star}$ Ignacio Muñoz-Sanjuan, $₫$ Celia Dominguez. $* *$

* Corresponding author

${ }^{\wedge}$ Equal contributors to this publication

$\dagger$ Charles River, Chesterford Research Park, Saffron Walden, UK CB10 1XL

$\ddagger$ CHDI Management/CHDI Foundation, 6080 Center Drive, Los Angeles, CA 90045, USA

\section{Contents}

Purification methods 3

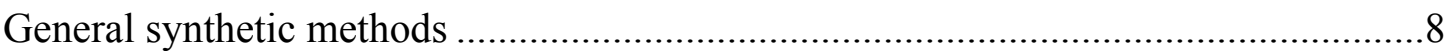

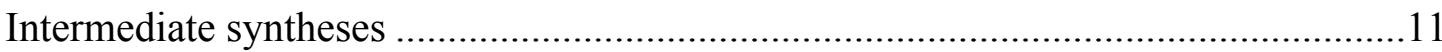

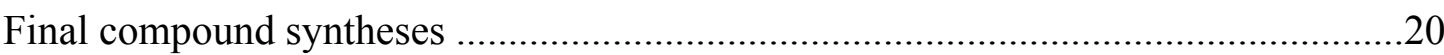

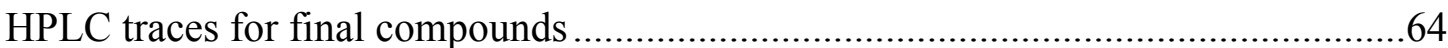

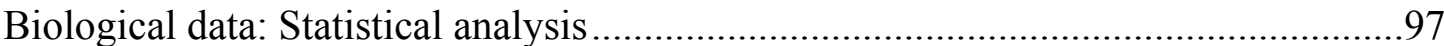

Table S1: Biological activity and statistical analysis for the synthesised ATM inhibitors.

Table S2: Mouse PK parameters for compound 55 after dosing at $2 \mathrm{mg} / \mathrm{kg} \mathrm{IV}, 10$ $\mathrm{mg} / \mathrm{kg} \mathrm{PO}$ and $30 \mathrm{mg} / \mathrm{kg} \mathrm{PO}$ 
Table S3: Details of data collection, processing and structure refinement statistics for the VPS34 ATM surrogate protein structure in complex with compound 44..101 


\section{Purification methods}

Preparative HPLC purification was performed by reverse phase HPLC using a Waters Fractionlynx $^{\text {TM }}$ preparative HPLC system (2525 pump, 2996/2998 UV/VIS detector, 2767 liquid handler) or an equivalent HPLC system such as a Gilson Trilution ${ }^{\circledR}$ UV directed system. The Waters ${ }^{\circledR} 2767$ liquid handler acted as both auto-sampler and fraction collector.

The columns used for the preparative purification of the compounds were a Waters Sunfire ${ }^{\circledR}$ OBD Phenomenex Luna ${ }^{\circledR}$ Phenyl Hexyl or Waters Xbridge ${ }^{\circledR}$ Phenyl at $10 \mu \mathrm{m} 19 \times 150 \mathrm{~mm}$ or Waters $\mathrm{CSH}^{\mathrm{TM}}$ Phenyl Hexyl, $19 \times 150,5 \mu \mathrm{m}$ column.

Appropriate focused gradients were selected based on acetonitrile and methanol solvent systems under either acidic or basic conditions. The modifiers used under acidic/basic conditions were formic acid or trifluoroacetic acid $(0.1 \% \mathrm{v} / \mathrm{v})$ and ammonium bicarbonate (10 $\mathrm{mM}$ ) respectively.

The purification was controlled by Waters Fractionlyn $x^{\mathrm{TM}}$ software through monitoring at 210$400 \mathrm{~nm}$ and triggered a threshold collection value at $260 \mathrm{~nm}$ and, when using the Fractionlynx ${ }^{\mathrm{TM}}$, the presence of target molecular ion as observed under APi conditions. Collected fractions were analysed by LCMS (Waters Acquity ${ }^{\circledR}$ systems with Waters ${ }^{\circledR}$ SQD).

\section{Analytical Method A:}

Analytical UPLC-MS was performed on a Waters Acquity I-Class UPLC with Waters Diode Array Detector (210-400 nm) coupled to a Waters SQD2 single quadrapole UPLC mass spectrometer using an HSS C18 column (1.8 $\mu \mathrm{m} 100 \times 2.1 \mathrm{~mm}$ plus guard). Method details are: 1) mobile phase: A: $0.1 \%$ formic acid $(\mathrm{v} / \mathrm{v})$ in water; $\mathrm{B}: 0.1 \%$ formic acid $(\mathrm{v} / \mathrm{v})$ in acetonitrile; 2) Gradient $0-1.2 \min 95 \%$ A 5\% B, 1.2 -3.5 min linear gradient to $0 \%$ A $100 \%$ 
B, 3.5-4.9 min $0 \%$ A $100 \% \mathrm{~B}, 4.9-5.0$ min gradient to $95 \%$ A $5 \%$ B, 5.0 min $-6.0 \min 95 \%$ A to $5 \% \mathrm{~B}$. 3) Flow rate $0.5 \mathrm{~mL} / \mathrm{min}$.

\section{Analytical Method B:}

Analytical UPLC-MS was performed on a Waters Acquity I-Class UPLC with Waters Diode Array Detector $(210-400 \mathrm{~nm})$ coupled to a Waters SQD2 single quadrapole UPLC mass spectrometer using a BEH Shield RP18 column (1.7 $\mu \mathrm{m} 100$ x $2.1 \mathrm{~mm}$. plus guard cartridge). Method details are: 1) mobile phase: A: $10 \mathrm{mM}$ ammonium bicarbonate in water; $\mathrm{B}$ : acetonitrile; 2) Gradient $0-1.2 \min 95 \%$ A 5\% B, $1.2-3.5$ min linear gradient to $0 \%$ A $100 \%$ $\mathrm{B}, 3.5-4.9 \min 0 \%$ A $100 \% \mathrm{~B}, 4.9-5.0$ min gradient to $95 \%$ A $5 \% \mathrm{~B}, 5.0 \mathrm{~min}-6.0 \mathrm{~min} 95 \%$ A to $5 \%$ B. 3) Flow rate $0.5 \mathrm{~mL} / \mathrm{min}$.

\section{Analytical Method C}

Analytical UPLC-MS was performed on a Waters UPLC with Waters Diode Array Detector (210-400 $\mathrm{nm}$ ) coupled to a Waters SQD2 single quadrapole UPLC mass spectrometer using a ACE-AR ACE excel column ( $2 \mu \mathrm{m}$ plus guard cartridge). Method details are: 1$)$ mobile phase: A: Water with $0.1 \%$ formic acid; B: Acetonitrile with $0.1 \%(v / v)$ formic acid; 2) Gradient 0 $1.2 \min 95 \%$ A $5 \%$ B, $1.2-3.5$ min linear gradient to $0 \%$ A $100 \%$ B, 3.5-4.9 min $0 \%$ A $100 \%$ B, $4.9-5.0$ min gradient to $95 \%$ A $5 \%$ B, $5.0 \min -6.0 \min 95 \%$ A to $5 \%$ B. 3) Flow rate 0.5 $\mathrm{mL} / \mathrm{min}$

\section{Analytical Method D}

Analytical UPLC-MS was performed on a Waters UPLC with Waters Diode Array Detector (210-400 nm) coupled to a Waters SQD2 single quadrapole UPLC mass spectrometer using a BEH Shield RP18 column (1.7 $\mu \mathrm{m} 100$ x $2.1 \mathrm{~mm}$. plus guard cartridge). Method details are: 1) mobile phase: A: Water with $10 \mathrm{mM}$ ammonium bicarbonate; B: Acetonitrile; 2) Gradient 
$0-1.2 \min 95 \%$ A $5 \%$ B, $1.2-3.5$ min linear gradient to $0 \%$ A $100 \% \mathrm{~B}, 3.5-4.9 \min 0 \% \mathrm{~A}$ $100 \% \mathrm{~B}, 4.9-5.0 \mathrm{~min}$ gradient to $95 \%$ A $5 \% \mathrm{~B}, 5.0 \mathrm{~min}-6.0 \mathrm{~min} 95 \%$ A to $5 \% \mathrm{~B}$. 3) Flow rate $0.5 \mathrm{~mL} / \mathrm{min}$.

\section{Analytical Method E}

Analytical UPLC-MS was performed on a Waters UPLC with Waters Diode Array Detector (210-400 $\mathrm{nm}$ ) coupled to a Waters SQD2 single quadrapole UPLC mass spectrometer using an Acquity UPLC HSS C18 $1.8 \mu \mathrm{m}$ column (100 x $2.1 \mathrm{~mm}$ plus guard cartridge). Method details are: 1) mobile phase: A: Water with $0.1 \%$ formic acid; B: Acetonitrile with $0.1 \%(\mathrm{v} / \mathrm{v})$ formic acid; 2) Gradient 0 - 1.2 min 95\% A 5\% B, 1.2 -3.5 min linear gradient to 0\% A 100\% B, 3.5$4.9 \min 0 \%$ A $100 \% \mathrm{~B}, 4.9-5.0 \mathrm{~min}$ gradient to $95 \% \mathrm{~A} 5 \% \mathrm{~B}, 5.0 \mathrm{~min}-6.0 \mathrm{~min} 95 \%$ A to 5\% B. 3) Flow rate $0.5 \mathrm{~mL} / \mathrm{min}$.

\section{Analytical Method F}

Analytical LCMS was performed on a Waters 2795/2695 separations module + Waters DAD + Micromass ZQ, single quadrapole LC-MS using a Waters Sunfire column $3.5 \mu \mathrm{m} \mathrm{4.6*50}$ $\mathrm{mm}$, maintained at $40{ }^{\circ} \mathrm{C}$. Detectors used were UV diode array 210-400 nm, Resolution 1.2 $\mathrm{nm}$, other wavelength traces are extracted from the DAD data. Method details are: 1) mobile phase A: Water + 0.1\% Formic Acid; B: Acetonitrile (Far UV grade) + 0.1\% Formic Acid; Gradient 0-0.5 min 95\% A, 0.5-3.5 min gradient to 5\% A, 3.5-4.5 min 5\% A, 4.5-4.6 min gradient to $95 \% \mathrm{~A}, 4.6-5.0 \mathrm{~min} 95 \% \mathrm{~A}$, Flow rate $2 \mathrm{~mL} / \mathrm{min}$.

\section{SFC methods:}

All compounds purified using Supercritical Fluid Chromatography (SFC) used either Waters Thar Prep100 preparative SFC system (P200 CO 2 pump, 2545 modifier pump, 2998 UV/VIS detector, 2767 liquid handler with Stacked Injection Module) or Waters Thar Investigator semi 
preparative system (Waters Fluid Delivery Module, 2998 UV/VIS detector, Waters Fraction Collection Module). Where the Waters 2767 liquid handler was used it acted as both autosampler and fraction collector.

The compounds were purified using an appropriate column from YMC Amylose-C, YMC Cellulose-C, Phenomenex LUX Cellulose-3 and Phenomenex LUX Cellulose-4. Appropriate isocratic methods were selected based on methanol, ethanol or isopropanol solvent systems under un-modified or basic conditions. The standard method used was modifier $/ \mathrm{CO}_{2}, 100$ $\mathrm{mL} / \mathrm{min}$ (or as appropriate), 120 bar backpressure, $40{ }^{\circ} \mathrm{C}$ column temperature where the specific modifier composition was as stated by the method development.

The modifier used under basic conditions was diethylamine $(0.1 \% \mathrm{v} / \mathrm{v})$. The purification was controlled either by Waters Fractionlynx or Waters Chromscope software through monitoring at 210-400 $\mathrm{nm}$ and triggered a threshold collection value at an appropriate wavelength. Collected fractions were analysed by SFC (Waters/Thar SFC systems with Waters SQD or Waters UPCC with Waters QDa). The fractions that contained the desired product were concentrated by vacuum centrifugation.

\section{Analytical Method SFC1}

Column

Column Dimensions

Mobile Phase

Gradient
See Specific Method $250 \times 4.6 \mathrm{~mm}$ id $5 \mathrm{um}$
$\mathrm{A}-\mathrm{CO}_{2}$
B - Primary Solvents
B - Secondary Solvents

Time (min) $\quad \% \mathrm{~B}$

0

5

0.5

5

3 55

4.5 55

4.6 5 


$\begin{array}{ll}\text { Temperature } & 40{ }^{\circ} \mathrm{C} \\ \text { Flow Rate } & 5.0 \mathrm{~mL} / \mathrm{min} \\ \text { System Back Pressure } & 120 \mathrm{bar} \\ \text { Detector } & \mathrm{DAD} 210-400 \mathrm{~nm} \\ \text { Injection Volume } & 5 \mu \mathrm{L}, \text { variable } \\ \text { MS } & \text { Electrospray }+/ \text {-ve ionization } \\ \text { Cone voltage } & 25 \mathrm{~V} \\ \text { Source Temperature } & 150{ }^{\circ} \mathrm{C} \\ \text { Mass Range } & 100-1000 \text { amu } \\ \text { Solvents and reagents used are } & \mathrm{HPLC} \text { gradient grade or equivalent } \\ \mathrm{CO}{ }_{2} \text { Grade } & 99.995 \% \\ \mathrm{~B}-\text { Primary Solvents } & \text { Methanol / Ethanol / Isopropanol } \\ \mathrm{B}-\text { Secondary Solvents } & \text { Methanol (+0.1\% Diethylamine }) / \text { Ethanol } \\ & (+0.1 \% \\ & (+0.1 \% \text { Diethylamine }) \text { or as specified }\end{array}$

\section{Analytical Method SFC4}

$\begin{array}{lll}\text { Column } & \text { See Specific Method } \\ \text { Column Dimensions } & 150 \times 2.1 \mathrm{~mm} \text { id } 5 \mathrm{um} \\ & 100 \times 3.0 \mathrm{~mm} \text { id } 1.7 \mathrm{um} \\ \text { Mobile Phase } & \mathrm{A}-\mathrm{CO}_{2} \\ & \mathrm{~B}-\text { Primary Solvents } \\ & \mathrm{B}-\text { Secondary Solvents } & \\ \text { Tradient } & 0 & \% \mathrm{~B} \\ & 0.5 & 5 \\ & 3 & 5 \\ & 4.5 & 55 \\ & 4.6 & 55 \\ \text { Temperature } & 5 & 5 \\ \text { Flow Rate } & 40{ }^{\circ} \mathrm{C} & 5\end{array}$




$\begin{array}{ll}\text { System Back Pressure } & 120 \mathrm{bar} \\ \text { Detector } & \text { DAD 210-400 nm } \\ \text { Injection Volume } & 2 \mu \mathrm{L}, \text { variable } \\ \text { MS } & \text { Electrospray }+/ \text {-ve ionization } \\ \text { Cone voltage } & 25 \mathrm{~V} \\ \text { Source Temperature } & 150{ }^{\circ} \mathrm{C} \\ \text { Mass Range } & 100-1000 \mathrm{amu} \\ \text { Solvents and reagents used are } \mathrm{HPLC} \text { gradient grade or equivalent } \\ \mathrm{CO}{ }_{2} \text { Grade } & 99.995 \% \\ \mathrm{~B}-\text { Primary Solvents } & \text { Methanol/Ethanol/Isopropanol } \\ \mathrm{B}-\text { Secondary Solvents } & \text { Methanol (+0.1\% Diethylamine }) / \text { Ethanol } \\ & (+0.1 \% \quad \text { Diethylamine }) / \text { Isopropanol } \\ & (+0.1 \% \text { Diethylamine) or as specified }\end{array}$

\section{General synthetic methods}

Method A: To a mixture of alcohol $(1.1 \mathrm{eq})$ in THF $(0.4 \mathrm{M})$ at $0{ }^{\circ} \mathrm{C}$ was added portionwise $\mathrm{NaH}$ ( 2 eq, $60 \%$ mineral oil). The reaction was stirred for $15 \mathrm{~min}$. Aryl chloride (1 eq) was added dropwise as a solution in THF $(0.7 \mathrm{M})$. After complete addition the reaction was warmed to r.t. and heated at reflux for $19 \mathrm{~h}$. The reaction mixture was cooled to $0{ }^{\circ} \mathrm{C}$ and cautiously quenched with water. The THF was removed under reduced pressure. The residue was dissolved in EtOAc and washed sequentially with water and brine. The organic extract was dried $\left(\mathrm{MgSO}_{4}\right)$, filtered and concentrated under reduced pressure. The crude product was either triturated or purified by silica gel column chromatography.

Method B: A mixture of amine (1.0 eq), 2,6-dichloro-4-iodopyridine (1.0 eq.), $\mathrm{NaO} t \mathrm{Bu}$ (2 eq) and $\mathrm{PdCl}_{2}\left(\mathrm{tBu}_{2} \mathrm{Pferrocene}\right)_{2}(0.025 \mathrm{eq})$ in toluene $(0.3 \mathrm{M})$ was placed in a sealed reaction tube under nitrogen and heated at $50{ }^{\circ} \mathrm{C}$ for $20 \mathrm{~h}$. The reaction mixture was cooled to $\mathrm{rt}$ and filtered 
through Celite. The mixture was concentrated under reduced pressure and purified by silica gel chromatography.

Method C: A suspension of aryl halide (1.0 eq), amine (1.0 eq) and $\mathrm{Cs}_{2} \mathrm{CO}_{3}$ (1.5 eq) in dry 1,4-dioxane $(0.2 \mathrm{M})$ was sparged with $\mathrm{N}_{2}$ for $15 \mathrm{~min}$. $\operatorname{Pd}_{2}(\mathrm{dba})_{3}(0.053 \mathrm{eq})$ and 2,2'bis(diphenylphosphino)-1,1'-binaphthyl ( 0.2 eq) were then added, the reaction tube was sealed under $\mathrm{N}_{2}$ and stirred at $100{ }^{\circ} \mathrm{C}$ for $16-40 \mathrm{~h}$. After cooling to r.t., the mixture was filtered through Celite, washing thoroughly with $\mathrm{CH}_{2} \mathrm{Cl}_{2}$ and the filtrate was concentrated.

Method D: For solid amines the following method was followed: A mixture of amine (1.0 1.4 eq), aryl halide (1.0 eq), $\mathrm{Cs}_{2} \mathrm{CO}_{3}$ or $\mathrm{NaO} t \mathrm{Bu}$ (1.1 eq), RuPhos (0.05-0.1 eq), and RuPhos palladacycle G1 methyl tert-butyl ether adduct (0.05-0.1eq) were placed in a stem block tube and sealed. The tube was evacuated and refilled with nitrogen (x3). 1,4-Dioxane (0.08-0.15 M) was added and the reaction heated at $85{ }^{\circ} \mathrm{C}$ for $16 \mathrm{~h}$. The reaction mixture was cooled to r.t. and filtered through Celite. The mixture was concentrated under reduced pressure.

In the case of the amine being an oil, it was introduced as a solution in dry 1,4-dioxane.

Method E: To a solution of PMB protected compound (1.0 eq) in ethanol $(0.15 \mathrm{M})$ was added $5 \%$ palladium on carbon ( $100 \%$ by weight of PMB compound) and the reaction mixture purged with $\mathrm{N}_{2}$ for 20 min. 1-Methylcyclohexa-1,4-diene (10 eq) was added and the reaction heated at $75{ }^{\circ} \mathrm{C}$ for $1-18 \mathrm{~h}$. The reaction was cooled to r.t. and filtered through Celite, washing with methanol. The solution was concentrated under reduced pressure.

Method F: TBAF hydrate (2.0 eq) was added to a solution of amine (1.08 eq) and sulfone (1.0 eq) in DMSO $(0.5 \mathrm{M})$ at r.t. The reaction mixture was heated at $80{ }^{\circ} \mathrm{C}$ for $18-70 \mathrm{~h}$. After this time the reaction was diluted with DCM and water and the layers were separated. 
The organic extracts were dried (phase separator) and concentrated under reduced pressure. Used without further purification in the next step.

Method G: TBAF hydrate (2.0 eq) was added to a solution of amine (1.08 eq.), sulfone (1.0 eq.) and DIPEA (2.0 eq) in DMSO $(0.5 \mathrm{M})$ at room temperature. The reaction mixture was heated at $80{ }^{\circ} \mathrm{C}$ for $18-70 \mathrm{~h}$. After this time the reaction was diluted with DCM and water and the layers were separated. The organic extracts were dried (phase separator) and concentrated under reduced pressure. Used without further purification in the next step.

Method H: TFA (0.24 M) was added to a solution of PMB-protected compound in DCM $(0.24 \mathrm{M})$. The reaction was stirred at r.t. for 1-4 hours before concentrating under reduced pressure. The residue was dissolved in DCM, washed with saturated sodium bicarbonate solution, dried (phase separator) and concentrated under reduced pressure. The crude material was purified by reverse phase preparative HPLC.

Method I: A stirred suspension of boronic acid/boronic ester (1 eq), aryl halide/triflate (1 eq) and $\mathrm{K}_{2} \mathrm{CO}_{3}\left(2.5\right.$ eq mmol) in dry dioxane $(0.15 \mathrm{M})$ was degassed. $\mathrm{Pd}\left(\mathrm{PPh}_{3}\right)_{4}(0.05$ eq $)$ was added and the reaction stirred at $100{ }^{\circ} \mathrm{C}$ for $16 \mathrm{~h}$. After cooling to r.t. the mixture was filtered through Celite, washing with $\mathrm{CH}_{2} \mathrm{Cl}_{2}$. The filtrate was concentrated and purified by silica gel chromatography.

Method J: A suspension of aryl halide ( $0.19 \mathrm{mmol}), \mathrm{tBu}_{3} \mathrm{PHBF}_{4}(0.75 \mathrm{eq})$, and $\mathrm{NaO} t \mathrm{Bu}(1 \mathrm{eq})$ in dry toluene $(0.1 \mathrm{M})$ was sparged with $\mathrm{N}_{2}$ for $20 \mathrm{~min} . \mathrm{Pd}(\mathrm{OAc})_{2}(0.2 \mathrm{eq})$, and amine $(1.1 \mathrm{eq})$ were then added, the reaction tube was sealed under $\mathrm{N}_{2}$ and stirred at $110^{\circ} \mathrm{C}$ for $16 \mathrm{~h}$. After cooling to r.t., the mixture was filtered through Celite, washing thoroughly with $\mathrm{CH}_{2} \mathrm{Cl}_{2}$. The filtrate was concentrated and the residue purified by silica gel chromatography. 
Method K: A stirred suspension of boronic acid (1.1 eq), aryl halide (1 eq) and $\mathrm{Na}_{2} \mathrm{CO}_{3}(2 \mathrm{M}$ aqueous solution, 3 eq) in dimethoxyethane $(0.15 \mathrm{M})$ was degassed. $\mathrm{Pd}\left(\mathrm{PPh}_{3}\right)_{4}(0.1 \mathrm{eq})$ was added and the reaction stirred at $85^{\circ} \mathrm{C}$ for $16 \mathrm{~h}$. The dimethoxyethane was then evaporated, and the residue was partitioned between $\mathrm{CH}_{2} \mathrm{Cl}_{2}$ and water. The organic was then passed through a phase separator, evaporated and purified by silica gel chromatography.

Method L: To a suspension of methoxyheteroaryl compound (1 eq) in acetonitrile ( $0.1 \mathrm{M})$ was added chlorotrimethylsilane ( 5 eq) followed by potassium iodide $(5 \mathrm{eq})$. The mixture was degassed by sparging with $\mathrm{N}_{2}$ for $20 \mathrm{~min}$, before being heated to $80{ }^{\circ} \mathrm{C}$ for $21 \mathrm{~h}$. The mixture was then cooled to r.t. and the solvent evaporated before being taken up with $\mathrm{CH}_{2} \mathrm{Cl}_{2}$ and washed with $\mathrm{Na}_{2} \mathrm{~S}_{2} \mathrm{O}_{3}$ aqueous solution. After passage through a phase separator and evaporation, the crude material was purified by normal phase HPLC or SFC.

\section{Intermediate syntheses}

(R)-2-Benzylazepane. Step 1: Tert-butyl (R)-(5-(2,2-dimethyl-4,6-dioxo-1,3-dioxan-5-yl)-5oxo-1-phenylpentan-2-yl)carbamate. (R)-4-((Tert-butoxycarbonyl)amino)-5-phenylpentanoic acid $(3.0 \mathrm{~g}, 10.2 \mathrm{mmol})$ was dissolved in DCM $(50 \mathrm{~mL})$ and cooled to $0{ }^{\circ} \mathrm{C}$. Meldrum's acid (1.47 g, $10.2 \mathrm{mmol})$, EDC hydrochloride (2.94 g, $15.3 \mathrm{mmol})$ and DMAP (1.87 g, $15.3 \mathrm{mmol})$ were added sequentially to the reaction. The reaction mixture was stirred for $19 \mathrm{~h}$, removing the ice-bath after $1 \mathrm{~h}$. The reaction was transferred to a separating funnel and washed with 1 M potassium bisulfate aqueous solution. The organic extracts were dried (phase separator) and concentrated under reduced pressure to afford the title compound. Used without further purification in the next step.

Step 2: Tert-butyl (R)-(5-(2,2-dimethyl-4,6-dioxo-1,3-dioxan-5-yl)-1-phenylpentan-2yl)carbamate. Tert-butyl $\quad(R)$-(5-(2,2-dimethyl-4,6-dioxo-1,3-dioxan-5-yl)-5-oxo-1- 
phenylpentan-2-yl)carbamate $(2.1 \mathrm{~g}, 5.01 \mathrm{mmol})$ was dissolved in DCM (50 mL) and cooled to $0{ }^{\circ} \mathrm{C}$. Acetic acid $(3.16 \mathrm{~mL}, 55.1 \mathrm{mmol})$ was added followed by portionwise addition of $\mathrm{NaBH}_{4}(759 \mathrm{mg}, 20.05 \mathrm{mmol})$. The reaction was stirred at $0{ }^{\circ} \mathrm{C}$ for $3.5 \mathrm{~h}$. The reaction mixture was cautiously quenched by dropwise addition of water $(5 \mathrm{~mL})$ followed by brine $(\sim 100 \mathrm{~mL})$. The layers were separated, and the aqueous layer extracted with DCM. The combined layers were washed with brine, dried $\left(\mathrm{MgSO}_{4}\right)$, filtered and concentrated under reduced pressure to afford the title compound. Used without further purification in the next step.

Step 3: Tert-butyl (R)-6-((tert-butoxycarbonyl)amino)-7-phenylheptanoate. Tert-butyl (R)-(5(2,2-dimethyl-4,6-dioxo-1,3-dioxan-5-yl)-1-phenylpentan-2-yl)carbamate (1.86 g, 4.59 mmol) was dissolved in toluene $(5 \mathrm{~mL})$ and $t-\mathrm{BuOH}(5 \mathrm{~mL})$. The reaction mixture was refluxed for 6 h. After this time the solvents were removed under reduced pressure. The resultant oil was dissolved in toluene and heated at reflux for $25 \mathrm{~h}$. The reaction mixture was concentrated under reduced pressure. The oil was dissolved in $\mathrm{DCM}$ and $\mathrm{NaHCO}_{3}$ (saturated aqueous solution) added. The layers were inseparable. A small quantity of brine was added to separate the layers. The aqueous layer was extracted with EtOAc. The combined organic layers were dried (phase separator) and concentrated under reduced pressure. The crude oil was purified by silica gel column chromatography (gradient elution $0-30 \%$ EtOAc/iso-hexane) to afford the title compound. ${ }^{1} \mathrm{H}$ NMR (400 MHz, $\left.\mathrm{CDCl}_{3}\right): \delta$ 7.32-7.14 $(5 \mathrm{H}, \mathrm{m}), 4.32(1 \mathrm{H}, \mathrm{d}, \mathrm{J}=0.8 \mathrm{~Hz}), 3.78$ $(1 \mathrm{H}$, br s), $3.32(2 \mathrm{H}, \mathrm{s}), 2.80-2.71(2 \mathrm{H}, \mathrm{m}), 2.60(2 \mathrm{H}, \mathrm{t}, \mathrm{J}=0.8 \mathrm{~Hz}), 1.83-1.81(1 \mathrm{H}, \mathrm{m}), 1.45$ $(9 \mathrm{H}, \mathrm{s}), 1.39(9 \mathrm{H}, \mathrm{s})$.

Step 4: (R)-6-Amino-7-phenylheptanoic acid. TFA $(2 \mathrm{~mL})$ was added to tert-butyl $(R)-6-((t e r t-$ butoxycarbonyl)amino)-7-phenylheptanoate (457 mg, $1.21 \mathrm{mmol})$ in DCM (2 mL) at r.t. with stirring. After $1.5 \mathrm{~h}$ the reaction mixture was concentrated under reduced pressure to afford a yellow oil. The oil was dissolved in $4 \mathrm{M} \mathrm{HCl}$ in dioxane $(10 \mathrm{~mL})$ and stirred at r.t. for $4 \mathrm{~h}$. The reaction mixture was concentrated under reduced pressure and dissolved in $4 \mathrm{M} \mathrm{HCl}$ in dioxane 
$(10 \mathrm{~mL})$. After $6 \mathrm{~h}$ the reaction mixture was concentrated under reduced pressure and used without further purification in the next step.

Step 5: (R)-7-Benzylazepan-2-one. EDC (348 mg, $1.82 \mathrm{mmol})$ and HOPO (201 mg, $1.82 \mathrm{mmol})$ were added to a solution of $(R)$-6-amino-7-phenylheptanoic acid $(1.21 \mathrm{mmol})$ and triethylamine $(1.01 \mathrm{~mL}, 7.26 \mathrm{mmol})$ in DMF $(13 \mathrm{~mL})$ at r.t. with stirring. After $54.5 \mathrm{~h}$ the reaction mixture was transferred to a separating funnel and diluted with DCM. The reaction mixture was washed sequentially with $1 \mathrm{M} \mathrm{HCl}(\mathrm{aq}), \mathrm{NaHCO}_{3}$ (saturated aqueous solution) and brine. The organics were dried $\left(\mathrm{MgSO}_{4}\right)$, filtered and concentrated under reduced pressure. The residue was purified by silica gel column chromatography (gradient elution 50-70\% EtOAc/iso-hexane) to afford the title compound. ${ }^{1} \mathrm{H} \mathrm{NMR}\left(400 \mathrm{MHz}, \mathrm{CDCl}_{3}\right): \delta 7.34-7.17(5 \mathrm{H}, \mathrm{m}), 5.54(1 \mathrm{H}$, brs), 3.68-3.55 (1H, m), 2.87-2.72 (2H, m), 2.51-2.38 (2H, m), 1.98-1.81 (3H, m), 1.61-1.32 (3H, $\mathrm{m})$.

Step 6: (R)-2-Benzylazepane. Lithium aluminium hydride $(0.59 \mathrm{~mL}, 0.59 \mathrm{mmol}, 1 \mathrm{M}$ solution in THF) was added dropwise to an ice-cooled solution of ( $R$ )-7-benzylazepan-2-one (60 $\mathrm{mg}$, $0.30 \mathrm{mmol})$ in THF $(2 \mathrm{~mL})$. The reaction mixture was warmed to r.t. and heated at reflux for $3 \mathrm{~h}$. The reaction mixture was cooled to r.t., followed by $0{ }^{\circ} \mathrm{C}$ and cautiously quenched with water. The THF was removed under reduced pressure. The mixture was diluted with water and EtOAc. The layers were separated and the organic extract dried $\left(\mathrm{MgSO}_{4}\right)$, filtered and concentrated under reduced pressure. The resultant oil was purified by SCX cartridge. The sample was loaded in DCM and eluted with 3 column volumes of DCM, 3 column volumes of methanol and 3 column volumes $7 \mathrm{~N} \mathrm{NH}_{3}$ in $\mathrm{MeOH} / \mathrm{DCM}(1: 9)$. The basic fractions were combined and concentrated under reduced pressure to afford the title compound. Used without further purification in the next step.

4-(2-Chloro-6-((4-methoxybenzyl)oxy)pyridin-4-yl)morpholine. Step $\quad$ 1: 4-(2,6Dichloropyridin-4-yl)morpholine. 2,6-Dichloropyridin-4-amine (10 g, $61.3 \mathrm{mmol})$ was 
dissolved in DMF $(200 \mathrm{~mL})$ and cooled to $0^{\circ} \mathrm{C}$. Sodium hydride $(6.13 \mathrm{~g}, 153 \mathrm{mmol})$ was added portionwise over $20 \mathrm{~min}$. After $15 \mathrm{~min}$ 1-chloro-2-(2-ethoxy)ethane (8.63 mL, $73.62 \mathrm{mmol})$ was added dropwise. The reaction mixture was warmed to r.t. and stirred for $21.5 \mathrm{~h}$. After this time the reaction was cooled to $0{ }^{\circ} \mathrm{C}$ and cautiously quenched with water. The DMF was removed under reduced pressure. The resultant residue was dissolved in DCM and washed with water. The organic layers were dried $\left(\mathrm{MgSO}_{4}\right)$, filtered and concentrated under reduced pressure. The crude product was triturated with EtOAc and iso-hexane to afford the title compound as a white solid (9.27 g, 65\%). ${ }^{1} \mathrm{H}$ NMR (400 MHz, $\left.\mathrm{CDCl}_{3}\right): \delta 6.59(2 \mathrm{H}, \mathrm{s}), 3.84$ $3.80(4 \mathrm{H}, \mathrm{m}), 3.33-3.29(4 \mathrm{H}, \mathrm{m})$

Step 2: 4-(2-Chloro-6-((4-methoxybenzyl)oxy)pyridin-4-yl)morpholine. Following Method A using 4-(2,6-dichloropyridin-4-yl)morpholine (9.27 g, $39.8 \mathrm{mmol})$ and para-methoxybenzyl alcohol $(5.43 \mathrm{~mL}, 43.8 \mathrm{mmol})$. The crude product was triturated with EtOAc and iso-hexane to afford the title compound. ${ }^{1} \mathrm{H}$ NMR $\left(400 \mathrm{MHz} \mathrm{CDCl}_{3}\right): \delta 7.39-7.35(2 \mathrm{H}, \mathrm{m}), 6.91-6.88$ $(2 \mathrm{H}, \mathrm{m}), 6.40(1 \mathrm{H}, \mathrm{d}, \mathrm{J}=2.0 \mathrm{~Hz}), 5.99(1 \mathrm{H}, \mathrm{d}, \mathrm{J}=2.0 \mathrm{~Hz}), 5.25(2 \mathrm{H}, \mathrm{s}), 3.81-3.77(7 \mathrm{H}, \mathrm{m}), 3.25$ $-3.21(4 \mathrm{H}, \mathrm{m})$.

(R)-4-(2-Chloro-6-((4-methoxybenzyl)oxy)pyridin-4-yl)-2-methylmorpholine (Intermediate 42). Step 1: (R)-4-(2,6-Dichloropyridin-4-yl)-2-methylmorpholine (Intermediate 38). Following Method B using (2R)-methyl morpholine (0.5 g, $5 \mathrm{mmol})$. Purified by silica gel column chromatography (gradient elution, 10-50\% EtOAc/iso-hexane) to afford the title compound. ${ }^{1} \mathrm{H}$ NMR (400 MHz, $\left.\mathrm{CDCl}_{3}\right): \delta 6.57(2 \mathrm{H}, \mathrm{s}), 4.02(1 \mathrm{H}, \mathrm{ddt}, \mathrm{J}=1.3,5.1,5.8 \mathrm{~Hz}), 3.74-$ $3.63(2 \mathrm{H}, \mathrm{m}), 3.58-3.49(2 \mathrm{H}, \mathrm{m}), 3.03(1 \mathrm{H}, \mathrm{dt}, \mathrm{J}=4.4,12.4 \mathrm{~Hz}), 2.67$ (1H, dd, J=10.4, $12.4 \mathrm{~Hz})$, $1.26(3 \mathrm{H}, \mathrm{d}, 6.4 \mathrm{~Hz})$.

Step 2: (R)-4-(2-Chloro-6-((4-methoxybenzyl)oxy)pyridin-4-yl)-2-methylmorpholine

Following Method A using (R)-4-(2,6-dichloropyridin-4-yl)-2-methylmorpholine (1.6 g, 6.4 
mmol) and para-methoxybenzyl alcohol $(0.87 \mathrm{~mL}, 7.0 \mathrm{mmol})$. Purified by silica gel column chromatography (gradient elution, 5-40\% EtOAc/iso-hexane) to afford the title compound. ${ }^{1} \mathrm{H}$ NMR (400 MHz, $\left.\mathrm{CDCl}_{3}\right): \delta 7.39$ - $7.36(2 \mathrm{H}, \mathrm{m}), 6.91-6.88(2 \mathrm{H}, \mathrm{m}), 6.40(1 \mathrm{H}, \mathrm{d}, \mathrm{J}=2.0 \mathrm{~Hz})$, $5.98(1 \mathrm{H}, \mathrm{d}, \mathrm{J}=2.0 \mathrm{~Hz}), 5.24(2 \mathrm{H}, \mathrm{s}), 4.00-3.95(1 \mathrm{H}, \mathrm{m}), 3.81(3 \mathrm{H}, \mathrm{s}), 3.71-3.61(2 \mathrm{H}, \mathrm{m})$, $3.52-3.42(2 \mathrm{H}, \mathrm{m}), 2.96-2.88(1 \mathrm{H}, \mathrm{m}), 2.58(1 \mathrm{H}, \mathrm{dd}, \mathrm{J}=10.4,12.4 \mathrm{~Hz}), 1.24-1.21(3 \mathrm{H}, \mathrm{m})$. $\left(1 R^{*}, 2 R^{*}, 5 S^{*}\right)-2-$ Benzyl-3-azabicyclo[3.1.0]hexane and $\left(1 R^{*}, 2 S^{*}, 5 S^{*}\right)-2-b e n z y l-3-$ azabicyclo[3.1.0]hexane.

Step 1: Tert-butyl 3-azabicyclo[3.1.0]hexane-3-carboxylate. Boc anhydride (2.6 g, $11.9 \mathrm{mmol})$ and a solution of $\mathrm{NaHCO}_{3}(3.32 \mathrm{~g}, 39.5 \mathrm{mmol})$ in water $(78 \mathrm{~mL})$ was added to a suspension of the 3-azabicyclo[3.1.0]hexane hydrochloride $(950 \mathrm{mg}, 7.9 \mathrm{mmol})$ in THF $(26 \mathrm{~mL})$. The resulting biphasic mixture was stirred vigorously overnight at r.t. The reaction mixture was diluted with water and DCM. The layers were separated and the organic phase dried (phase separator) and concentrated under reduced pressure. The residue was purified by silica gel column chromatography (gradient elution, $0-10 \%$ EtOAc/iso-hexane) to afford the title compound as a mixture of rotamers. ${ }^{1} \mathrm{H}$ NMR $\left(400 \mathrm{MHz}, \mathrm{CDCl}_{3}\right): \delta 3.51(2 \mathrm{H}$, dd, J=10.9, 36.1 Hz), 3.35-3.27 (2H, m), $1.42(9 \mathrm{H}, \mathrm{s}), 1.21-1.14(1 \mathrm{H}, \mathrm{m}), 0.93-0.84(1 \mathrm{H}, \mathrm{m}), 0.67-0.61(1 \mathrm{H}, \mathrm{m})$, $0.16-0.13(1 \mathrm{H}, \mathrm{m})$.

Step 2: Tert-butyl $\left(1 R^{*}, 2 R^{*}, 5 S^{*}\right)$-2-benzyl-3-azabicyclo[3.1.0]hexane-3-carboxylate and tertbutyl $\left(1 R^{*}, 2 S^{*}, 5 S^{*}\right)$-2-benzyl-3-azabicyclo[3.1.0]hexane-3-carboxylate. To a solution of tertbutyl 3-azabicyclo[3.1.0]hexane-3-carboxylate $(1.50 \mathrm{~g}, 7.9 \mathrm{mmol})$ in diethyl ether $(27 \mathrm{~mL})$, under $\mathrm{N}_{2}$ atmosphere, was added tetramethylethylenediamine $(1.23 \mathrm{~mL}, 8.2 \mathrm{mmol})$ and the reaction mixture cooled to $-78{ }^{\circ} \mathrm{C}$. $\mathrm{sec}$-BuLi $(11.8 \mathrm{~mL}, 10.6 \mathrm{mmol}, 0.9 \mathrm{M}$ in cyclohexane) was added and the mixture was stirred at $-78^{\circ} \mathrm{C}$ for $30 \mathrm{~min}$. Benzyl bromide (1.95 mL, $\left.16.4 \mathrm{mmol}\right)$ was added dropwise at $-78{ }^{\circ} \mathrm{C}$. After complete addition the reaction was allowed to slowly 
warm to r.t. overnight. The reaction was quenched with saturated aqueous $\mathrm{NH}_{4} \mathrm{Cl}$ solution, the layers separated and the aqueous further extracted with $\mathrm{Et}_{2} \mathrm{O}(\mathrm{x} 2)$. The combined organic extracts were dried $\left(\mathrm{MgSO}_{4}\right)$ and concentrated under reduced pressure. The crude material was purified by silica gel column chromatography (gradient elution, 0-10\% EtOAc/iso-hexane) to afford the title compounds as separate diastereomers tert-butyl $\left(1 R^{*}, 2 S^{*}, 5 S^{*}\right)-2$-benzyl-3azabicyclo[3.1.0]hexane-3-carboxylate and tert-butyl $\left(1 R^{*}, 2 R^{*}, 5 S^{*}\right)$-2-benzyl-3azabicyclo[3.1.0]hexane-3-carboxylate.

Isomer A (592 mg): LCMS (ES $\left.{ }^{+}\right) 218\left(\mathrm{M}-{ }^{\mathrm{t}} \mathrm{Bu}\right)^{+}$, RT $1.77 \mathrm{~min}$ (Analytical Method F); ${ }^{1} \mathrm{H}$ NMR (400 MHz, $\left.\mathrm{CDCl}_{3}\right): \delta$ 7.31-7.18 (5H, m), 4.12-3.96 (1H, m), 3.61-3.39 (1H, m), 3.22-2.91 (2H, m), 2.77-2.64 (1H, m), 1.48-1.42 (9H, m), 1.38-1.28 (1H, m), 0.95-0.87 $(1 \mathrm{H}, \mathrm{m}), 0.59-0.53$ $(1 \mathrm{H}, \mathrm{m}), 0.12-0.09(1 \mathrm{H}, \mathrm{m})$, rotamers observed.

Isomer B (420 mg): LCMS (ES $\left.{ }^{+}\right) 218\left(\mathrm{M}-{ }^{\mathrm{t}} \mathrm{Bu}\right)^{+}$, RT 1.83 min (Analytical Method F); ${ }^{1} \mathrm{H}$ NMR $\left(400 \mathrm{MHz}, \mathrm{CDCl}_{3}\right): \delta$ 7.33-7.27 (4H, m), 7.23-7.17 (1H, m), 4.04-3.95 (1H, m), 3.86-3.44 (3H, m), $2.40(1 \mathrm{H}, \mathrm{dd}, \mathrm{J}=10.8,12.4 \mathrm{~Hz}), 1.57-1.34(11 \mathrm{H}, \mathrm{m}), 0.69-0.63(1 \mathrm{H}, \mathrm{m}), 0.47-0.43(1 \mathrm{H}, \mathrm{m})$, rotamers observed.

Step 3: $\left(1 R^{*}, 2 R^{*}, 5 S^{*}\right)-2-B e n z y l-3$-azabicyclo[3.1.0]hexane. To tert-butyl $\left(1 R^{*}, 2 R^{*}, 5 S^{*}\right)$-2benzyl-3-azabicyclo[3.1.0]hexane-3-carboxylate $(570 \mathrm{mg}, 2.09 \mathrm{mmol})$ was added DCM (5 $\mathrm{mL})$ and TFA $(5 \mathrm{~mL})$ and the resulting mixture was stirred at r.t. for $1 \mathrm{~h}$. The solvent was evaporated and purified by SCX cartridge eluting sequentially with DCM:MeOH, 1:1 then 3:1 DCM:7N ammonia in methanol. The ammonia in methanol fractions were combined and concentrated under reduced pressure to afford the title compound. nOe experiments allowed determination of the stereochemistry of the benzyl group relative to the cyclopropyl group. ${ }^{1} \mathrm{H}$ NMR (400 MHz, $\left.\mathrm{CDCl}_{3}\right): \delta 7.32-7.17(5 \mathrm{H}, \mathrm{m}), 3.41(1 \mathrm{H}, \mathrm{ddd}, \mathrm{J}=3.0,5.5,8.1 \mathrm{~Hz}), 2.94(2 \mathrm{H}$, 
d, J=1.8 Hz), $2.82(1 \mathrm{H}, \mathrm{dd}, \mathrm{J}=5.9,13.0 \mathrm{~Hz}), 2.61(1 \mathrm{H}, \mathrm{dd}, \mathrm{J}=7.8,13.1 \mathrm{~Hz}), 1.38-1.32(1 \mathrm{H}, \mathrm{m})$, $1.30-1.23(1 \mathrm{H}, \mathrm{m}), 0.40-0.34(1 \mathrm{H}, \mathrm{m}), 0.34-0.30(1 \mathrm{H}, \mathrm{m}) . \mathrm{NH}$ not observed.

Step 4: $\left(1 R^{*}, 2 S^{*}, 5 S^{*}\right)-2-B e n z y l-3$-azabicyclo[3.1.0]hexane. To tert-butyl $\left(1 R^{*}, 2 S^{*}, 5 S^{*}\right)-2-$ benzyl-3-azabicyclo[3.1.0]hexane-3-carboxylate (400 mg, $1.46 \mathrm{mmol})$ was added DCM (3.5 $\mathrm{mL})$ and TFA $(3.5 \mathrm{~mL})$ and the resulting mixture was stirred at r.t. for $1 \mathrm{~h}$. The solvent was evaporated and purified by SCX cartridge eluting sequentially with DCM:MeOH, 1:1 then 3:1 DCM:7N ammonia in methanol. The ammonia in methanol fractions were combined and concentrated under reduced pressure to afford the title compound. nOe experiments allowed determination of the stereochemistry of the benzyl group relative to the cyclopropyl group. ${ }^{1} \mathrm{H}$ NMR (400 MHz, $\left.\mathrm{CDCl}_{3}\right): \delta 7.32-7.17(5 \mathrm{H}, \mathrm{m}), 3.29(1 \mathrm{H}, \mathrm{t}, \mathrm{J}=7.2 \mathrm{~Hz}), 3.00(1 \mathrm{H}, \mathrm{dd}, \mathrm{J}=3.2$, 11.2 Hz), $2.87(1 \mathrm{H}, \mathrm{d}, \mathrm{J}=11.0 \mathrm{~Hz}), 2.69(1 \mathrm{H}, \mathrm{dd}, \mathrm{J}=7.2,13.5 \mathrm{~Hz}), 2.60(1 \mathrm{H}, \mathrm{dd}, \mathrm{J}=7.5,13.4 \mathrm{~Hz})$, $1.43-1.36(1 \mathrm{H}, \mathrm{m}), 1.29(1 \mathrm{H}, \mathrm{ddd}, \mathrm{J}=4.0,6.2,7.9 \mathrm{~Hz}), 0.45(1 \mathrm{H}, \mathrm{dt}, \mathrm{J}=5.0,7.7 \mathrm{~Hz}), 0.15(1 \mathrm{H}$, dd, J=4.0, $8.7 \mathrm{~Hz})$. NH not observed.

4-(6-((4-Methoxybenzyl)oxy)-2-(methylsulfonyl)pyrimidin-4-yl)morpholine

Step 1: 4-(6-((4-Methoxybenzyl)oxy)-2-(methylthio)pyrimidin-4-yl)morpholine. NaH (3.43 g, $85.8 \mathrm{mmol}, 60 \%$ dispersion in mineral oil) was suspended in DMF (70 mL) and cooled to 0 ${ }^{\circ} \mathrm{C}$. 4-methoxy benzyl alcohol (6.52 g, $\left.47.2 \mathrm{mmol}\right)$ in DMF $(30 \mathrm{~mL})$ was added dropwise to the suspension over 15 minutes. After stirring at $0{ }^{\circ} \mathrm{C}$ for $15 \mathrm{~min}$ 4-(6-chloro-2(methylthio)pyrimidin-4-yl)morpholine (10.54 g, $42.9 \mathrm{mmol}$, prepared according to WO 2008/125839) in DMF (90 mL). The reaction was allowed to warm to r.t. and stirred for 4.5 h. The reaction was cooled to $0{ }^{\circ} \mathrm{C}$ and cautiously quenched with water. The reaction mixture was concentrated under reduced pressure. The residue was dissolved in EtOAc and washed with brine and water. The organic extracts were dried $\left(\mathrm{MgSO}_{4}\right)$, filtered and concentrated under reduced pressure. Purified by silica gel column chromatography (gradient elution, 10- 
50\% EtOAc/iso-hexane) to afford the title compound. LCMS $\left(\mathrm{ES}^{+}\right) 348(\mathrm{M}+\mathrm{H})^{+}, \mathrm{RT} 1.69 \mathrm{~min}$ (Analytical Method F); ${ }^{1} \mathrm{H}$ NMR (400 MHz, $\left.\mathrm{CDCl}_{3}\right)$ : $\delta$ 7.37-7.33 (2H, m), 6.91-6.87 (2H, m), $5.53(1 \mathrm{H}, \mathrm{s}), 5.30(2 \mathrm{H}, \mathrm{s}), 3.80(3 \mathrm{H}, \mathrm{s}), 3.76-3.72(4 \mathrm{H}, \mathrm{m}), 3.55-3.51(4 \mathrm{H}, \mathrm{m}), 2.51(3 \mathrm{H}, \mathrm{s})$.

Step 2: 4-(6-((4-Methoxybenzyl)oxy)-2-(methylsulfonyl)pyrimidin-4-yl)morpholine. m-CPBA (759 mg, $4.4 \mathrm{mmol}, 50-55$ wt.\% in $\left.\mathrm{H}_{2} \mathrm{O}\right)$ was added portionwise to a solution of 4-(6-((4methoxybenzyl)oxy)-2-(methylthio)pyrimidin-4-yl)morpholine (695 mg, $2.0 \mathrm{mmol}$ ) in DCM $(20 \mathrm{~mL})$ at r.t. After $2 \mathrm{~h}$ the reaction was filtered, washing the precipitate with DCM. The filtrate was washed successively with saturated $\mathrm{NaHCO}_{3}$ (aqueous solution), brine, dried (phase separator) and concentrated under reduced pressure to afford the product, which was used without further purification in the next step. ${ }^{1} \mathrm{H}$ NMR $\left(400 \mathrm{MHz}, \mathrm{CDCl}_{3}\right): \delta 7.38-7.35$ $(2 \mathrm{H}, \mathrm{m}), 6.91-6.88(2 \mathrm{H}, \mathrm{m}), 5.88(1 \mathrm{H}, \mathrm{s}), 5.36(2 \mathrm{H}, \mathrm{s}), 3.81(3 \mathrm{H}, \mathrm{s}), 3.78-3.74(4 \mathrm{H}, \mathrm{m}), 3.63$ - $3.57(4 \mathrm{H}, \mathrm{m}), 3.26(3 \mathrm{H}, \mathrm{s})$.

(R)-4-(6-((4-Methoxybenzyl)oxy)-2-(methylsulfonyl)pyrimidin-4-yl)-2-methylmorpholine. Step 1: 4-Chloro-6-((4-methoxybenzyl)oxy)-2-(methylthio)pyrimidine. A solution of 4,6-dichloro-2(methylthio)pyrimidine $(15.0 \mathrm{~g}, 77.0 \mathrm{mmol})$ in dry DMF $(205 \mathrm{~mL})$ was treated at r..t. with para-methoxybenzyl alcohol (11.7 g, $84.6 \mathrm{mmol})$ and $\mathrm{K}_{2} \mathrm{CO}_{3}(42.6 \mathrm{~g}, 308 \mathrm{mmol})$. The reaction was stirred at $60^{\circ} \mathrm{C}$ for $64 \mathrm{~h}$. After cooling to r.t., the mixture was diluted with water $(700 \mathrm{~mL})$ and stirred vigorously at r.t. for 2 h. The solids that formed were collected by filtration, washing with water, to give the title compound. ${ }^{1} \mathrm{H}$ NMR (400 MHz, $\left.\mathrm{CDCl}_{3}\right): \delta 7.35(2 \mathrm{H}, \mathrm{d}$, $\mathrm{J}=8.8 \mathrm{~Hz}), 6.92-6.89(2 \mathrm{H}, \mathrm{m}), 6.42(1 \mathrm{H}, \mathrm{s}), 5.35(2 \mathrm{H}, \mathrm{s}), 3.81(3 \mathrm{H}, \mathrm{s}), 2.56(3 \mathrm{H}, \mathrm{s})$.

Step 2: (R)-4-(6-((4-Methoxybenzyl)oxy)-2-(methylthio)pyrimidin-4-yl)-2-methylmorpholine. A solution of 4-chloro-6-((4-methoxybenzyl)oxy)-2-(methylthio)pyrimidine (11.3 g, 38.0 mmol) in dry THF $(95 \mathrm{~mL})$ was treated with DIPEA $(20 \mathrm{~mL}, 114 \mathrm{mmol})$ and $(2 R)$ methylmorpholine hydrochloride $(5.75 \mathrm{~g}, 41.8 \mathrm{mmol})$. The mixture was stirred at $65^{\circ} \mathrm{C}$ for 16 
h. After cooling to r.t., the reaction mixture was poured into water. DCM was added, and the layers were separated by passage through a hydrophobic frit. The DCM layer was concentrated and purified by silica gel column chromatography (gradient elution, 0-25\% EtOAc/iso-hexane) to give the title compound. ${ }^{1} \mathrm{H}$ NMR $\left(400 \mathrm{MHz} \mathrm{CDCl}_{3}\right): \delta 7.36-7.33(2 \mathrm{H}, \mathrm{m}), 6.90-6.87$ $(2 \mathrm{H}, \mathrm{m}), 5.53(1 \mathrm{H}, \mathrm{s}), 5.30(2 \mathrm{H}, \mathrm{s}), 4.09$ - $3.91(3 \mathrm{H}, \mathrm{m}), 3.81(3 \mathrm{H}, \mathrm{s}), 3.64-3.54(2 \mathrm{H}, \mathrm{m}), 2.96$ $(1 \mathrm{H}, \mathrm{ddd}, \mathrm{J}=3.6,11.9,13.0 \mathrm{~Hz}), 2.61(1 \mathrm{H}, \mathrm{dd}, \mathrm{J}=10.5,13.0 \mathrm{~Hz}), 2.51(3 \mathrm{H}, \mathrm{s}), 1.22(3 \mathrm{H}, \mathrm{d}, \mathrm{J}=6.5$ $\mathrm{Hz})$.

Step 3: $\quad$ (R)-4-(6-((4-Methoxybenzyl)oxy)-2-(methylsulfonyl)pyrimidin-4-yl)-2methylmorpholine. A solution of (R)-4-(6-((4-methoxybenzyl)oxy)-2-(methylthio)pyrimidin4-yl)-2-methylmorpholine $(9.37 \mathrm{~g}, 25.9 \mathrm{mmol})$ in DCM $(172 \mathrm{~mL})$ was treated with $m$-CPBA (19.7 g, $57.0 \mathrm{mmol}, 50-55 \mathrm{wt} . \%$ in $\mathrm{H}_{2} \mathrm{O}$ ) at $\mathrm{rt}$ and the mixture was stirred at r.t. for $4 \mathrm{~h}$. The solid impurity was then filtered off and the organic filtrate was shaken with $\mathrm{NaHCO}_{3}$ saturated solution $(2 \mathrm{x})$. The organic phase was dried (phase separator) and concentrated under reduced pressure. The residue was purified by silica gel column chromatography (gradient elution, 0$65 \%$ EtOAc/iso-hexane) to give the title compound. ${ }^{1} \mathrm{H}$ NMR (400 MHz, $\left.\mathrm{CDCl}_{3}\right): \delta 7.36(2 \mathrm{H}$, d, J=8.7 Hz), $6.90(2 \mathrm{H}, \mathrm{d}, \mathrm{J}=8.7 \mathrm{~Hz}), 5.88(1 \mathrm{H}, \mathrm{s}), 5.35(2 \mathrm{H}, \mathrm{s}), 4.14-4.02(2 \mathrm{H}, \mathrm{m}), 3.98(1 \mathrm{H}$, ddd, J=1.4, 3.7, $11.8 \mathrm{~Hz}), 3.81(3 \mathrm{H}, \mathrm{s}), 3.69$ - $3.55(2 \mathrm{H}, \mathrm{m}), 3.26(3 \mathrm{H}, \mathrm{s}), 3.07$ (1H, ddd, J=3.7, 11.9, $13.0 \mathrm{~Hz}), 2.71(1 \mathrm{H}, \mathrm{dd}, \mathrm{J}=10.5,13.0 \mathrm{~Hz}), 1.24(3 \mathrm{H}, \mathrm{d}, \mathrm{J}=6.4 \mathrm{~Hz})$.

$\left(2 R^{*}, \quad 3 S^{*}\right)-2-B e n z y l-3-m e t h y l p y r r o l i d i n e . \quad$ Step $\quad 1: \quad$ Tert-butyl $\quad\left(2 R^{*}, 3 S^{*}\right)-2-b e n z y l-3-$ methylpyrrolidine-1-carboxylate. Palladium (II) acetate (18 mg, $0.08 \mathrm{mmol}$, Dpe-phos (86 mg, $0.16 \mathrm{mmol})$ and sodium tert-butoxide $(884 \mathrm{mg}, 9.2 \mathrm{mmol})$ were placed in a reaction tube sealed, evacuated and re-filled with nitrogen (x3). 3-Methylpent-4-en-1-yl)carbamate (798 mg, 4.0 mmol) was dissolved in dioxane $(16 \mathrm{~mL})$ and degassed. The solution was added to the reaction tube followed by bromobenzene $(0.25 \mathrm{~mL}, 4.8 \mathrm{mmol})$ and the reaction heated at $100{ }^{\circ} \mathrm{C}$ for 
17.5 h. The reaction mixture was cooled to r.t., diluted with $\mathrm{NH}_{4} \mathrm{Cl}$. The reaction mixture was extracted with EtOAc (x3). The organic extracts dried (phase separator) and concentrated under reduced pressure. The crude product was purified by silica gel column chromatography (gradient elution, 2-20\% EtOAc/iso-hexane) to afford the title compound. ${ }^{1} \mathrm{H}$ NMR $(400 \mathrm{MHz}$, $\left.\mathrm{CDCl}_{3}\right): \delta 7.31-7.13(5 \mathrm{H}, \mathrm{m}), 3.68-3.36(2 \mathrm{H}, \mathrm{m}), 3.27-2.98(2 \mathrm{H}, \mathrm{m}), 2.79-2.57(1 \mathrm{H}, \mathrm{m}), 2.11-$ $1.76(2 \mathrm{H}, \mathrm{m}), 1.61-1.46(9 \mathrm{H}, \mathrm{m}), 0.95-0.81(4 \mathrm{H}, \mathrm{m})$, rotamers observed.

Step 2: $\quad\left(2 R^{*}, 3 S^{*}\right)$-2-Benzyl-3-methylpyrrolidine. $\quad$ Tert-butyl $\quad\left(2 R^{*}, 3 S^{*}\right)-2$-benzyl-3methylpyrrolidine-1-carboxylate (785 $\mathrm{mg}, 2.85 \mathrm{mmol}$ ) was dissolved in $4 \mathrm{M} \mathrm{HCl}$ in dioxane $(7.1 \mathrm{~mL})$ at r.t. with stirring. After $2 \mathrm{~h}$ the reaction mixture was concentrated under reduced pressure. The residue was dissolved in DCM and washed with saturated $\mathrm{NaHCO}_{3}$ solution. The organic extract was dried (phase separator) and concentrated under reduced pressure to afford the title compound. ${ }^{1} \mathrm{H} \mathrm{NMR}\left(400 \mathrm{MHz}, \mathrm{CDCl}_{3}\right): \delta 7.32-7.16(5 \mathrm{H}, \mathrm{m}), 3.00-2.79(3 \mathrm{H}$, m), 2.77 - $2.68(1 \mathrm{H}, \mathrm{m}), 2.59(1 \mathrm{H}, \mathrm{dd}, \mathrm{J}=8.5,13.3 \mathrm{~Hz}), 2.08-1.96(1 \mathrm{H}, \mathrm{m}), 1.76-1.66(1 \mathrm{H}$, m), 1.42 - $1.31(1 \mathrm{H}, \mathrm{m}), 1.00(3 \mathrm{H}, \mathrm{d}, \mathrm{J}=6.6 \mathrm{~Hz}), \mathrm{NH}$ not observed.

\section{Final compound syntheses}

6-(7-Methoxy-9H-thioxanthen-4-yl)-4-morpholino-1H-pyridin-2-one (7).

Step 1: 4-Chloro-6-(7-methoxy-9H-thioxanthen-4-yl)-1H-pyridin-2-one (Intermediate 6). Following Method I from 4,6-dichloro-1H-pyridin-2-one (255 mg, $1.55 \mathrm{mmol}$ ) and 2-(7methoxy-9H-thioxanthen-4-yl)-4,4,5,5-tetramethyl-1,3,2-dioxaborolane ${ }^{9}$ (549 mg, 1.55 mmol). Purification by silica gel chromatography (gradient elution; $0-75 \%$ ethyl acetate in iso-hexane) gave $78 \mathrm{mg}(14 \%)$ of slightly impure title compound. LCMS (ES+) 356, 358 $(\mathrm{M}+\mathrm{H})^{+}$, RT $2.96 \min \left(\right.$ Analytical Method F); ${ }^{1} \mathrm{H}$ NMR $\left(400 \mathrm{MHz}, \mathrm{CDCl}_{3}\right): \delta 10.82(1 \mathrm{H}, \mathrm{s})$, 
7.42-7.39 (1H, m), 7.31-7.23 (3H, m), $6.92(1 \mathrm{H}, \mathrm{d}, \mathrm{J}=2.4 \mathrm{~Hz}), 6.75(1 \mathrm{H}, \mathrm{dd}, \mathrm{J}=2.4,8.3 \mathrm{~Hz})$, $6.55(1 \mathrm{H}, \mathrm{J}=1.7 \mathrm{~Hz}), 6.35(1 \mathrm{H}, \mathrm{d}, \mathrm{J}=1.7 \mathrm{~Hz}), 3.87(2 \mathrm{H}, \mathrm{s}), 3.81(3 \mathrm{H}, \mathrm{s})$.

Step 2: 6-(7-Methoxy-9H-thioxanthen-4-yl)-4-morpholino-1H-pyridin-2-one (7). A suspension of 4-chloro-6-(7-methoxy-9H-thioxanthen-4-yl)-1H-pyridin-2-one (69 $\mathrm{mg}, 0.19 \mathrm{mmol}$ ), $t \mathrm{Bu}_{3} \mathrm{PHBF}_{4}$ (44 mg, $\left.0.15 \mathrm{mmol}\right)$, and $\mathrm{NaO} t \mathrm{Bu}(88 \mathrm{mg}, 0.91 \mathrm{mmol})$ in dry toluene $(2 \mathrm{~mL})$ was sparged with $\mathrm{N}_{2}$ for $20 \mathrm{~min}$. $\mathrm{Pd}(\mathrm{OAc})_{2}(8.5 \mathrm{mg}, 0.038 \mathrm{mmol})$, and morpholine (19 $\mu \mathrm{L}, 0.21$ mmol) were then added, the reaction tube was sealed under $\mathrm{N}_{2}$ and stirred at $110{ }^{\circ} \mathrm{C}$ for $16 \mathrm{~h}$. After cooling to r.t., the mixture was filtered through Celite, washing thoroughly with $\mathrm{CH}_{2} \mathrm{Cl}_{2}$. The filtrate was concentrated, and the residue purified by silica gel chromatography (gradient elution; $0-5 \%$ methanol in $\mathrm{CH}_{2} \mathrm{Cl}_{2}$ ) to give impure title compound. Further purification by normal phase HPLC yielded $28 \mathrm{mg}$ of the title compound as a solid (36\%). LCMS (ES $\left.{ }^{+}\right) 407$ $(\mathrm{M}+\mathrm{H})^{+}$, RT $3.19 \min \left(\right.$ Analytical Method C); ${ }^{1} \mathrm{H}$ NMR (400 MHz, $\left.\mathrm{CDCl}_{3}\right): \delta 8.84(1 \mathrm{H}$, br s), $7.40(1 \mathrm{H}, \mathrm{dd}, \mathrm{J}=1.9,6.8 \mathrm{~Hz}), 7.26(3 \mathrm{H}, \mathrm{m}), 6.92(1 \mathrm{H}, \mathrm{d}, \mathrm{J}=2.6 \mathrm{~Hz}), 6.76(1 \mathrm{H}, \mathrm{dd}, \mathrm{J}=2.8,8.5$ Hz), $6.01(1 \mathrm{H}, \mathrm{d}, \mathrm{J}=2.8 \mathrm{~Hz}), 5.72(1 \mathrm{H}, \mathrm{d}, \mathrm{J}=2.4 \mathrm{~Hz}), 3.87(2 \mathrm{H}, \mathrm{s}), 3.84-3.80(7 \mathrm{H}, \mathrm{m}), 3.35$ $3.31(4 \mathrm{H}, \mathrm{m})$. HRMS $\left(\mathrm{ESI}^{+}\right)(\mathrm{M}+\mathrm{H})^{+}$: calcd for $\mathrm{C}_{23} \mathrm{H}_{23} \mathrm{~N}_{2} \mathrm{O}_{3} \mathrm{~S}, 407.1429$; found, 407.1436.

Following Method J from 4-chloro-6-(7-methoxy-9H-thioxanthen-4-yl)-1H-pyridin-2-one $(0.19 \mathrm{mmol})$ and $(S)$-2-methylmorpholine $(0.21 \mathrm{mmol}) . \quad \mathrm{LCMS}_{\left(\mathrm{ES}^{+}\right)} 421(\mathrm{M}+\mathrm{H})^{+}, \mathrm{RT} 3.15$ min (Analytical Method E); ${ }^{1} \mathrm{H}$ NMR (400 MHz, DMSO-d 6 ): $\delta 11.00(1 \mathrm{H}, \mathrm{s}), 7.54(1 \mathrm{H}, \mathrm{dd}$, $\mathrm{J}=1.5,7.3 \mathrm{~Hz}), 7.43-7.31(3 \mathrm{H}, \mathrm{m}), 7.13(1 \mathrm{H}, \mathrm{d}, \mathrm{J}=2.5 \mathrm{~Hz}), 6.85(1 \mathrm{H}, \mathrm{dd}, \mathrm{J}=2.5,8.5 \mathrm{~Hz}), 6.08$ $(1 \mathrm{H}, \mathrm{s}), 5.59$ - $5.55(1 \mathrm{H}, \mathrm{m}), 3.96-3.90(3 \mathrm{H}, \mathrm{m}), 3.81(4 \mathrm{H}, \mathrm{s}), 3.71-3.55(3 \mathrm{H}, \mathrm{m}), 2.86(1 \mathrm{H}$, td, J=12.2, $\left.3.5 \mathrm{~Hz}), 2.54-2.51(1 \mathrm{H}, \mathrm{m}), 1.17(3 \mathrm{H}, \mathrm{d}, \mathrm{J}=6.3 \mathrm{~Hz}) . \mathrm{HRMS}_{(\mathrm{ESI}}{ }^{+}\right)(\mathrm{M}+\mathrm{H})^{+}$: calcd for $\mathrm{C}_{24} \mathrm{H}_{25} \mathrm{~N}_{2} \mathrm{O}_{3} \mathrm{~S}$, 421.1586; found, 421.1586 . 
6-(7-Methoxy-9H-thioxanthen-4-yl)-1-methyl-4-morpholino-pyridin-2-one (11). Step 1: 4,6Dichloro-1-methyl-pyridin-2-one (Intermediate 9). 4,6-Dichloro-1H-pyridin-2-one (100 mg, $0.61 \mathrm{mmol})$ was dissolved in acetone $(3 \mathrm{~mL})$. Methyl iodide $(75 \mu \mathrm{L}, 1.2 \mathrm{mmol})$ and potassium carbonate $(249 \mathrm{mg}, 1.8 \mathrm{mmol})$ were added to the mixture and stirred at r.t. for $19 \mathrm{~h}$. The insoluble material was filtered off, washed with acetone and the filtrate evaporated to afford $109 \mathrm{mg}$ (quant.) of a 3.2:1 mixture of title compound as the major component together with 2,4-dichloro-6-methoxy-pyridine (structures confirmed by nOe experiments). LCMS (ES ${ }^{+}$) 177, $179(\mathrm{M}+\mathrm{H})^{+}$, RT 2.96 min (Analytical Method F); major isomer: ${ }^{1} \mathrm{H}$ NMR $(400 \mathrm{MHz}$, $\left.\mathrm{CDCl}_{3}\right): \delta 6.55(1 \mathrm{H}, \mathrm{d}, \mathrm{J}=2.2 \mathrm{~Hz}), 6.36(1 \mathrm{H}, \mathrm{d}, \mathrm{J}=2.2 \mathrm{~Hz}), 3.64(3 \mathrm{H}, \mathrm{s})$; minor isomer: ${ }^{1} \mathrm{H}$ NMR $\left(400 \mathrm{MHz}, \mathrm{CDCl}_{3}\right): 6.93(1 \mathrm{H}, \mathrm{d}, \mathrm{J}=1.0 \mathrm{~Hz}), 6.68(1 \mathrm{H}, \mathrm{d}, \mathrm{J}=1.0 \mathrm{~Hz}), 3.94(3 \mathrm{H}, \mathrm{s})$.

Step 2: 4-Chloro-6-(7-methoxy-9H-thioxanthen-4-yl)-1-methyl-pyridin-2-one (Intermediate 10). Following Method I from the mixture of 4,6-dichloro-1-methyl-pyridin-2-one and 2,4dichloro-6-methoxy-pyridine from the previous step $(100 \mathrm{mg})$ and 2-(7-methoxy-9Hthioxanthen-4-yl)-4,4,5,5-tetramethyl-1,3,2-dioxaborolane ${ }^{9}$ (200 mg, $0.56 \mathrm{mmol}$ ). Purification by silica gel chromatography (gradient elution, 0-100\% EtOAc/iso-hexane) gave $74 \mathrm{mg}$ (47\% yield, corrected for isomer contamination of SM) of the title compound (structures confirmed by nOe experiments). LCMS (ES $) 370(\mathrm{M}+\mathrm{H})^{+}$, RT $3.2 \min$ (Analytical Method F); ${ }^{1} \mathrm{H}$ NMR $\left(400 \mathrm{MHz}, \mathrm{CDCl}_{3}\right): \delta 7.44(1 \mathrm{H}, \mathrm{d}, \mathrm{J}=8.4 \mathrm{~Hz}), 7.33-7.26(2 \mathrm{H}, \mathrm{m}), 7.12(1 \mathrm{H}, \mathrm{dd}, \mathrm{J}=1,7.5 \mathrm{~Hz})$, 6.93-6.90 (1H, m), $6.77(1 \mathrm{H}, \mathrm{dd}, \mathrm{J}=2.2,8.4 \mathrm{~Hz}), 6.72(1 \mathrm{H}, \mathrm{d}, \mathrm{J}=2.2 \mathrm{~Hz}), 6.18(1 \mathrm{H}, \mathrm{d}, \mathrm{J}=2.2$ $\mathrm{Hz}), 3.83-3.81(5 \mathrm{H}, \mathrm{m}), 3.18(1 \mathrm{H}, \mathrm{s})$.

Step 3: 6-(7-Methoxy-9H-thioxanthen-4-yl)-1-methyl-4-morpholino-pyridin-2-one (11). Following Method J from 4-chloro-6-(7-methoxy-9H-thioxanthen-4-yl)-1-methyl-pyridin-2one (72 $\mathrm{mg}, 0.195 \mathrm{mmol})$ and morpholine $(19 \mu \mathrm{L}, 0.21 \mathrm{mmol})$. Purification by normal phase HPLC yielded $14 \mathrm{mg}$ of the title compound as a solid (17\%). LCMS (ES $\left.{ }^{+}\right) 421(\mathrm{M}+\mathrm{H})^{+}, \mathrm{RT}$ $3.19 \min$ (Analytical Method D); ${ }^{1} \mathrm{H}$ NMR (400 MHz, $\left.\mathrm{CDCl}_{3}\right): \delta 7.41(1 \mathrm{H}, \mathrm{d}, \mathrm{J}=7.1 \mathrm{~Hz}), 7.32$ 
- $7.28(2 \mathrm{H}, \mathrm{m}), 7.12(1 \mathrm{H}, \mathrm{d}, \mathrm{J}=7.1 \mathrm{~Hz}), 6.93(1 \mathrm{H}, \mathrm{d}, \mathrm{J}=2.3 \mathrm{~Hz}), 6.76(1 \mathrm{H}, \mathrm{dd}, \mathrm{J}=2.3,8.5 \mathrm{~Hz})$, $5.88-5.86(2 \mathrm{H}, \mathrm{m}), 3.98(1 \mathrm{H}, \mathrm{d}, \mathrm{J}=15.4 \mathrm{~Hz}), 3.82-3.77(8 \mathrm{H}, \mathrm{m}), 3.30-3.24(4 \mathrm{H}, \mathrm{m}), 3.13$ $(3 \mathrm{H}, \mathrm{s})$. HRMS $\left(\mathrm{ESI}^{+}\right)(\mathrm{M}+\mathrm{H})^{+}$: calcd for $\mathrm{C}_{24} \mathrm{H}_{25} \mathrm{~N}_{2} \mathrm{O}_{3} \mathrm{~S}, 421.1586$; found, 421.1585 .

2-(7-Methoxy-9H-thioxanthen-4-yl)-6-morpholino-pyridin-4-ol (16). Step 1: 4-(4-Benzyloxy6-chloro-2-pyridyl)morpholine (Intermediate 14). 4-Benzyloxy-2,6-dichloro-pyridine (1.00 g, $5.58 \mathrm{mmol}$ ), which was synthesised following the literature protocol (WO2011007856) was dissolved in 1,4-dioxane $(10 \mathrm{~mL})$ and morpholine $(171 \mu \mathrm{L}, 6.0 \mathrm{mmol})$ was added. The mixture was heated to $110^{\circ} \mathrm{C}$ for $18 \mathrm{~h}$, after which additional morpholine $(350 \mu \mathrm{L}, 12.0 \mathrm{mmol})$ was added and the mixture heated for $24 \mathrm{~h}$, after which the solvent was evaporated to dryness. Purification by silica gel chromatography (gradient elution, 0-25\% EtOAc/iso-hexane) gave $430 \mathrm{mg}(70 \%)$ of the title compound. LCMS (ES $) 305(\mathrm{M}+\mathrm{H})^{+}$, RT $3.35 \mathrm{~min}$ (Analytical Method F).

Step 2: 4-[4-Benzyloxy-6-(7-methoxy-9H-thioxanthen-4-yl)-2-pyridyl]morpholine (Intermediate 15). Following Method K from 4-(4-benzyloxy-6-chloro-2-pyridyl)morpholine $(0.1 \mathrm{~g}, \quad 0.33 \mathrm{mmol})$ and 2-(7-methoxy-9H-thioxanthen-4-yl)-4,4,5,5-tetramethyl-1,3,2dioxaborolane $^{9}(117 \mathrm{mg}, 0.33 \mathrm{mmol})$ to obtain the crude material. Purification by silica gel chromatography (gradient elution, 0-30\% EtOAc/iso-hexane) gave $96 \mathrm{mg}$ (57\%) of the title compound. LCMS (ES $\left.{ }^{+}\right) 497(\mathrm{M}+\mathrm{H})^{+}$, RT 3.69 min (Analytical Method F); ${ }^{1} \mathrm{H}$ NMR (400 $\left.\mathrm{MHz}, \mathrm{CDCl}_{3}\right): \delta$ 7.45-7.31 (7H, m), 7.24-7.22 (3H, m), $6.89(1 \mathrm{H}, \mathrm{d}, \mathrm{J}=3 \mathrm{~Hz}), 6.69(1 \mathrm{H}, \mathrm{dd}$, $\mathrm{J}=3.0,8.1 \mathrm{~Hz}), 6.63(1 \mathrm{H}, \mathrm{d}, \mathrm{J}=1.5 \mathrm{~Hz}), 5.14(2 \mathrm{H}, \mathrm{s}), 3.88-3.84(6 \mathrm{H}, \mathrm{m}), 3.78(3 \mathrm{H}, \mathrm{s}), 3.66-3.63$ $(4 \mathrm{H}, \mathrm{m})$.

Step 3: 2-(7-Methoxy-9H-thioxanthen-4-yl)-6-morpholino-pyridin-4-ol (16). 4-[4-Benzyloxy6-(7-methoxy-9H-thioxanthen-4-yl)-2-pyridyl]morpholine (90 mg, $0.18 \mathrm{mmol}$ ) in methanol $(10 \mathrm{~mL})$ was hydrogenated in an $\mathrm{H}$-cube using $\mathrm{Pd} / \mathrm{C}$, in controlled mode, at r.t. Purification by silica gel chromatography (gradient elution, $0-4 \% \mathrm{NH}_{3}$ in $\mathrm{MeOH}(7 \mathrm{~N}) / \mathrm{CH}_{2} \mathrm{Cl}_{2}$ ), then by $\mathrm{SFC}$, 
followed by freeze drying from acetonitrile/water gave $33 \mathrm{mg}(45 \%)$ as a colorless solid. LCMS $\left(\mathrm{ES}^{+}\right) 407(\mathrm{M}+\mathrm{H})^{+}$, RT 3.07 min (Analytical Method A); ${ }^{1} \mathrm{H}$ NMR (400 MHz, DMSO-d 6 ): $\delta$ $10.45(1 \mathrm{H}, \mathrm{s}), 7.48-7.43(2 \mathrm{H}, \mathrm{m}), 7.32(2 \mathrm{H}, \mathrm{dd}, \mathrm{J}=8.0,15.3 \mathrm{~Hz}), 7.10(1 \mathrm{H}, \mathrm{d}, \mathrm{J}=2.8 \mathrm{~Hz}), 6.80$ $(1 \mathrm{H}, \mathrm{dd}, \mathrm{J}=2.8,8.5 \mathrm{~Hz}), 6.47(1 \mathrm{H}, \mathrm{d}, \mathrm{J}=1.5 \mathrm{~Hz}), 6.20(1 \mathrm{H}, \mathrm{d}, \mathrm{J}=1.5 \mathrm{~Hz}), 3.93(2 \mathrm{H}, \mathrm{s}), 3.80(3 \mathrm{H}$, s), 3.79 - $3.75(4 \mathrm{H}, \mathrm{m}), 3.57-3.52(4 \mathrm{H}, \mathrm{m})$. HRMS $\left(\mathrm{ESI}^{+}\right)(\mathrm{M}+\mathrm{H})^{+}$: calcd for $\mathrm{C}_{23} \mathrm{H}_{23} \mathrm{~N}_{2} \mathrm{O}_{3} \mathrm{~S}$, 407.1429; found, 407.143.

4-(7-Methoxy-9H-thioxanthen-4-yl)-6-morpholino-1H-pyridin-2-one (19). Step 1: 4-Iodo-6morpholino-1H-pyridin-2-one (Intermediate 18). 4-(6-Chloro-4-iodo-2-pyridyl)morpholine (300 mg, $0.92 \mathrm{mmol}$, synthesized following the literature protocol in WO2012122383) was suspended in $\mathrm{tBuOH}(3.1 \mathrm{~mL})$. Freshly powdered $\mathrm{KOH}(86 \mathrm{mg}, 1.54 \mathrm{mmol})$ was added and the mixture was heated to $82^{\circ} \mathrm{C}$ for $5 \mathrm{~h}$. Additional freshly powdered $\mathrm{KOH}(300 \mathrm{mg}, 5.3 \mathrm{mmol}$ ) was added and the mixture was heated to $82{ }^{\circ} \mathrm{C}$ for $92 \mathrm{~h}$. Additional freshly powdered $\mathrm{KOH}$ (300 mg, $5.3 \mathrm{mmol}$ ) was added and the mixture was heated to $82{ }^{\circ} \mathrm{C}$ for $20 \mathrm{~h}$. After cooling to r.t., the mixture was diluted with EtOAc and washed with water. The aqueous phase was acidified to $\mathrm{pH}$ 1-2 and extracted with EtOAc (x2). All organic phases were combined and purification by silica gel chromatography (gradient elution, 0-100\% EtOAc/iso-hexane) gave $52 \mathrm{mg}(18 \%)$ of the title compound. LCMS $\left(\mathrm{ES}^{+}\right) 325(\mathrm{M}+\mathrm{H})^{+}$, RT $3.32 \mathrm{~min}$ (Analytical Method F).

Step 2: 4-(7-Methoxy-9H-thioxanthen-4-yl)-6-morpholino-1H-pyridin-2-one (19). Following Method K from 4-iodo-6-morpholino-1 $H$-pyridin-2-one $(51 \mathrm{mg}, 0.17 \mathrm{mmol})$ and 2-(7methoxy-9H-thioxanthen-4-yl)-4,4,5,5-tetramethyl-1,3,2-dioxaborolane ${ }^{9}$ (72 mg, $\left.0.26 \mathrm{mmol}\right)$ to obtain the crude material. Purification by silica gel chromatography (gradient elution, $0-4 \%$ $7 \mathrm{~N} \mathrm{NH}_{3}$ in $\mathrm{MeOH} / \mathrm{CH}_{2} \mathrm{Cl}_{2}$ ), followed by freeze drying from acetonitrile/water gave $42 \mathrm{mg}$ (61\% yield) as an off-white solid. LCMS (ES $\left.{ }^{+}\right) 407(\mathrm{M}+\mathrm{H})^{+}$, RT $3.07 \mathrm{~min}$ (Analytical Method E); ${ }^{1} \mathrm{H}$ NMR $\left(400 \mathrm{MHz}, \mathrm{CDCl}_{3}\right): \delta 10.90(1 \mathrm{H}, \mathrm{s}), 7.34(1 \mathrm{H}, \mathrm{d}, \mathrm{J}=7.3 \mathrm{~Hz}), 7.28(1 \mathrm{H}, \mathrm{d}, \mathrm{J}=8.5$ 
Hz), 7.25 - $7.18(2 \mathrm{H}, \mathrm{m}), 6.91(1 \mathrm{H}, \mathrm{d}, \mathrm{J}=2.6 \mathrm{~Hz}), 6.74(1 \mathrm{H}, \mathrm{dd}, \mathrm{J}=2.6,8.5 \mathrm{~Hz}), 6.05(1 \mathrm{H}, \mathrm{s})$,

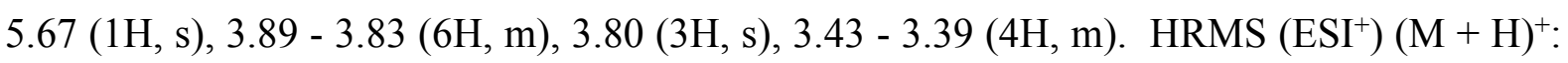
calcd for $\mathrm{C}_{23} \mathrm{H}_{23} \mathrm{~N}_{2} \mathrm{O}_{3} \mathrm{~S}, 407.1429$; found, 407.1427.

2-(7-Methoxy-9H-thioxanthen-4-yl)-6-morpholino-pyrimidin-4-ol (25) and 6-(7-Methoxy-9Hthioxanthen-4-yl)-2-morpholino-pyrimidin-4-ol (26). Step 1: 4-(4-Chloro-6-methoxypyrimidin-2-yl)morpholine and 4-(2-chloro-6-methoxy-pyrimidin-4-yl)morpholine. 2,4Dichloro-6-methoxy-pyrimidine $(1.00 \mathrm{~g}, 5.58 \mathrm{mmol})$ was dissolved in DMF (11 mL) before morpholine (488 $\mu \mathrm{L}, 5.4 \mathrm{mmol})$ and $\mathrm{K}_{2} \mathrm{CO}_{3}(1.54 \mathrm{~g}, 11.20 \mathrm{mmol})$ were added. The mixture was stirred at r.t for $17 \mathrm{~h}$, after which the inorganics were filtered off and washed with EtOAc. The filtrate was evaporated to dryness and partitioned between $\mathrm{CH}_{2} \mathrm{Cl}_{2}$ and $4 \%$ aqueous $\mathrm{LiCl}$ solution. The organic was passed through a phase separator and evaporated. Purification by normal phase HPLC gave $858 \mathrm{mg}$ (67\%) of 4-(4-chloro-6-methoxy-pyrimidin-2-yl)morpholine 21 together with $106 \mathrm{mg}$ (8\%) of 4-(2-chloro-6-methoxy-pyrimidin-4-yl)morpholine 22 (structures confirmed by nOe experiments).

Intermediate 21: $\mathrm{LCMS}_{\left(\mathrm{ES}^{+}\right)} 230(\mathrm{M}+\mathrm{H}){ }^{+}, \mathrm{RT} 3.10 \mathrm{~min}$ (Analytical Method F); ${ }^{1} \mathrm{H}$ NMR $(400$ $\left.\mathrm{MHz}, \mathrm{CDCl}_{3}\right): \delta 6.02(1 \mathrm{H}, \mathrm{s}), 3.89(3 \mathrm{H}, \mathrm{s}), 3.81-3.71(8 \mathrm{H}, \mathrm{m})$.

Intermediate 22: $\mathrm{LCMS}\left(\mathrm{ES}^{+}\right) 230(\mathrm{M}+\mathrm{H}){ }^{+}, \mathrm{RT} 2.69 \min$ (Analytical Method F); ${ }^{1} \mathrm{H}$ NMR $(400$ $\left.\mathrm{MHz}, \mathrm{CDCl}_{3}\right): \delta 5.67(1 \mathrm{H}, \mathrm{s}), 3.92(3 \mathrm{H}, \mathrm{s}), 3.77-3.74(4 \mathrm{H}, \mathrm{m}), 3.58-3.53(4 \mathrm{H}, \mathrm{m})$.

Step 2a: 4-[4-methoxy-6-(7-methoxy-9H-thioxanthen-4-yl)pyrimidin-2-yl]morpholine. Following Method K from 4-(4-chloro-6-methoxy-pyrimidin-2-yl)morpholine ( $0.1 \mathrm{~g}, 0.44$ mmol) and 2-(7-methoxy-9H-thioxanthen-4-yl)-4,4,5,5-tetramethyl-1,3,2-dioxaborolane ${ }^{9}$ (155 $\mathrm{mg}, 0.44 \mathrm{mmol}$ ) and purified by silica gel chromatography (gradient elution, 0-20\% EtOAc/isohexane) to give $119 \mathrm{mg}$ of target material. $30 \mathrm{mg}$ was then further purified further by SFC to yield $23 \mathrm{mg}$ (equivalent to $49 \%$ yield when extrapolated to whole crude) of 4-[4-methoxy-6- 
(7-methoxy-9H-thioxanthen-4-yl)pyrimidin-2-yl]morpholine, after freeze drying from acetonitrile-water. LCMS (ES $\left.{ }^{+}\right) 422(\mathrm{M}+\mathrm{H})^{+}$, RT $4.01 \min$ (Analytical Method E); ${ }^{1} \mathrm{H}$ NMR (400 MHz, DMSO-d 6 ): $\delta 7.55(2 \mathrm{H}, \mathrm{dd}, \mathrm{J}=2.7,7.5 \mathrm{~Hz}), 7.40-7.32(2 \mathrm{H}, \mathrm{m}), 7.10(1 \mathrm{H}, \mathrm{d}, \mathrm{J}=2.7$ Hz), $6.81(1 \mathrm{H}, \mathrm{dd}, \mathrm{J}=2.7,8.5 \mathrm{~Hz}), 6.41(1 \mathrm{H}, \mathrm{s}), 3.98(3 \mathrm{H}, \mathrm{s}), 3.95(2 \mathrm{H}, \mathrm{s}), 3.92$ - $3.86(4 \mathrm{H}, \mathrm{m})$, $3.80(3 \mathrm{H}, \mathrm{s}), 3.79-3.74(4 \mathrm{H}, \mathrm{m})$.

Step 2b: 4-[6-Methoxy-2-(7-methoxy-9H-thioxanthen-4-yl)pyrimidin-4-yl]morpholine Following Method K from 4-(2-chloro-6-methoxy-pyrimidin-4-yl)morpholine (0.1 g, 0.44 mmol) and 2-(7-methoxy-9H-thioxanthen-4-yl)-4,4,5,5-tetramethyl-1,3,2-dioxaborolane ${ }^{9}$ (155 $\mathrm{mg}, 0.44 \mathrm{mmol}$ ) and purified by silica gel chromatography (gradient elution, 0-20\% EtOAc/isohexane) to give $83 \mathrm{mg}$ of target material. $20 \mathrm{mg}$ was then further purified by SFC to yield $9 \mathrm{mg}$ (equivalent to $20 \%$ yield when extrapolated to whole crude) of 4-[6-methoxy-2-(7-methoxy9H-thioxanthen-4-yl)pyrimidin-4-yl]morpholine, after freeze drying from acetonitrile-water. LCMS (ES $\left.{ }^{+}\right) 422(\mathrm{M}+\mathrm{H})^{+}$, RT 3.91 min (Analytical Method E); ${ }^{1} \mathrm{H}$ NMR (400 MHz, DMSO$\left.\mathrm{d}_{6}\right): \delta 7.95(1 \mathrm{H}, \mathrm{dd}, \mathrm{J}=1.4,7.7 \mathrm{~Hz}), 7.57-7.54(1 \mathrm{H}, \mathrm{m}), 7.41-7.33(2 \mathrm{H}, \mathrm{m}), 7.11(1 \mathrm{H}, \mathrm{d}, \mathrm{J}=2.5$ Hz), $6.82(1 \mathrm{H}, \mathrm{dd}, \mathrm{J}=2.5,8.5 \mathrm{~Hz}), 6.17(1 \mathrm{H}, \mathrm{s}), 4.02(3 \mathrm{H}, \mathrm{s}), 3.95(2 \mathrm{H}, \mathrm{s}), 3.81(3 \mathrm{H}, \mathrm{s}), 3.79$ $3.70(8 \mathrm{H}, \mathrm{m})$.

Step 3a: 2-(7-Methoxy-9H-thioxanthen-4-yl)-6-morpholino-pyrimidin-4-ol (25) Following Method L from 4-[6-methoxy-2-(7-methoxy-9H-thioxanthen-4-yl)pyrimidin-4-yl]morpholine (85 $\mathrm{mg}, 0.20 \mathrm{mmol})$ afforded material which was purified by SFC to yield $47 \mathrm{mg}(58 \%)$ of compound 25, after freeze drying from 1,4-dioxane-water. LCMS $\left(\mathrm{ES}^{+}\right) 408(\mathrm{M}+\mathrm{H})^{+}$, RT 3.31 min (Analytical Method E); ${ }^{1} \mathrm{H}$ NMR (400 MHz, DMSO-d $\left.{ }_{6}\right): \delta 11.48(1 \mathrm{H}, \mathrm{s}), 7.51(2 \mathrm{H}, \mathrm{dd}$, $\mathrm{J}=7.6,10.4 \mathrm{~Hz}), 7.35(2 \mathrm{H}, \mathrm{dd}, \mathrm{J}=8.1,8.1 \mathrm{~Hz}), 7.10(1 \mathrm{H}, \mathrm{d}, \mathrm{J}=2.5 \mathrm{~Hz}), 6.83(1 \mathrm{H}, \mathrm{dd}, \mathrm{J}=2.5,8.1$ Hz), $6.01(1 \mathrm{H}, \mathrm{s}), 3.93(2 \mathrm{H}, \mathrm{s}), 3.81-3.77(7 \mathrm{H}, \mathrm{m}), 3.77$ - $\left.3.72(4 \mathrm{H}, \mathrm{m}) . \mathrm{HRMS}_{(\mathrm{ESI}}{ }^{+}\right)(\mathrm{M}+$ $\mathrm{H})^{+}$: calcd for $\mathrm{C}_{22} \mathrm{H}_{22} \mathrm{~N}_{3} \mathrm{O}_{3} \mathrm{~S}, 408.1382$; found, 408.1381 . 
Step 3b: 6-(7-methoxy-9H-thioxanthen-4-yl)-2-morpholino-pyrimidin-4-ol (26) Following Method L from 4-[4-methoxy-6-(7-methoxy-9H-thioxanthen-4-yl)pyrimidin-2-yl]morpholine (60 mg, $0.14 \mathrm{mmol}$ ) afforded material which was purified by SFC to yield $57 \mathrm{mg}$ (quantitative) of compound 26, after freeze drying from 1,4-dioxane-water. LCMS $\left(\mathrm{ES}^{+}\right) 408(\mathrm{M}+\mathrm{H})^{+}, \mathrm{RT}$ 3.19 min (Analytical Method E); ${ }^{1} \mathrm{H}$ NMR (400 MHz, DMSO-d 6 ): $\delta 12.13(1 \mathrm{H}, \mathrm{s}), 7.60(2 \mathrm{H}$, d, J=7.3 Hz), $7.42-7.37(2 \mathrm{H}, \mathrm{m}), 7.12(1 \mathrm{H}, \mathrm{d}, \mathrm{J}=2.8 \mathrm{~Hz}), 6.84(1 \mathrm{H}, \mathrm{dd}, \mathrm{J}=2.8,8.6 \mathrm{~Hz}), 5.45$ $(1 \mathrm{H}, \mathrm{s}), 3.95(2 \mathrm{H}, \mathrm{s}), 3.81(3 \mathrm{H}, \mathrm{s}), 3.75(4 \mathrm{H}, \mathrm{t}, \mathrm{J}=4.7 \mathrm{~Hz}), 3.67$ - $3.62(4 \mathrm{H}, \mathrm{m})$. HRMS $\left(\mathrm{ESI}^{+}\right)$ $(\mathrm{M}+\mathrm{H})^{+}$: calcd for $\mathrm{C}_{22} \mathrm{H}_{22} \mathrm{~N}_{3} \mathrm{O}_{3} \mathrm{~S}, 408.1382$; found, 408.1379 .

(R)-6-(2-Benzylpyrrolidin-1-yl)-2-morpholinopyrimidin-4-ol (30). Step 1: 4,6-Dichloro-2((4-methoxybenzyl)oxy)pyrimidine and 2,4-dichloro-6-((4-methoxybenzyl)oxy)pyrimidine. A mixture of 2,4,6-trichloropyrimidine ( $9.17 \mathrm{~g}, 0.05 \mathrm{~mol})$, 4-methoxybenzyl alcohol (6.91 g, 0.05 mol) and $\mathrm{Cs}_{2} \mathrm{CO}_{3}(32.6 \mathrm{~g}, 0.1 \mathrm{~mol})$ in $\mathrm{MeCN}(150 \mathrm{~mL})$ was stirred at r.t. for $48 \mathrm{~h}$. The solvent was removed by evaporation and the residue partitioned between DCM (150 mL) and water $(50 \mathrm{~mL})$. The DCM fraction was concentrated to give the title compounds in a 2:1 molar ratio (identities not confirmed) as a yellow liquid (12.8 g), which was used without further purification.

Step 2: 4-(4-Chloro-6-((4-methoxybenzyl)oxy)pyrimidin-2-yl)morpholine. A mixture of 4,6dichloro-2-((4-methoxybenzyl)oxy)pyrimidine and 2,4-dichloro-6-((4methoxybenzyl)oxy)pyrimidine from step 1 (12.8 g), morpholine $(4.31 \mathrm{~mL}, 0.05 \mathrm{~mol})$ and $\mathrm{Cs}_{2} \mathrm{CO}_{3}(32.6 \mathrm{~g}, 0.1 \mathrm{~mol})$ in $\mathrm{MeCN}(150 \mathrm{~mL})$ was stirred at r.t. for $20 \mathrm{~h}$. The solvent was removed by evaporation and the residue partitioned between DCM $(250 \mathrm{~mL})$ and water $(50$ $\mathrm{mL}$ ). The DCM fraction was concentrated, and the residue was purified by silica gel column chromatography (gradient elution, 0-20\% EtOAc/iso-hexane) to give the title compound (7.76 g, $23 \mathrm{mmol}$, 46\% over two steps). All three morpholino isomers obtained from this reaction 
were separated by column chromatography, and their structure confirmed by nOe NMR experiments.

Intermediate 27: ${ }^{1} \mathrm{H}$ NMR (400 MHz, $\left.\mathrm{CDCl}_{3}\right): \delta$ 7.33-7.32 $(2 \mathrm{H}, \mathrm{m}), 6.91-6.88(2 \mathrm{H}, \mathrm{m}), 6.04$ $(1 \mathrm{H}, \mathrm{s}), 5.26(2 \mathrm{H}, \mathrm{s}), 3.82-3.74(11 \mathrm{H}, \mathrm{m})$.

Intermediate 28: ${ }^{1} \mathrm{H}$ NMR (400 MHz, $\left.\mathrm{CDCl}_{3}\right): \delta$ 7.37-7.34 (2H, m), 6.93-6.87 (2H, m), 5.70 $(1 \mathrm{H}, \mathrm{s}), 5.30(2 \mathrm{H}, \mathrm{s}), 3.81(3 \mathrm{H}, \mathrm{s}), 3.76-3.72(4 \mathrm{H}, \mathrm{m}), 3.56-3.51(4 \mathrm{H}, \mathrm{m})$.

Intermediate 29: ${ }^{1} \mathrm{H}$ NMR (400 MHz, $\left.\mathrm{CDCl}_{3}\right): \delta$ 7.39-7.35 (2H, m), 6.89-6.86 (2H, m), 6.17 $(1 \mathrm{H}, \mathrm{s}), 5.28(2 \mathrm{H}, \mathrm{s}), 3.73(3 \mathrm{H}, \mathrm{s}), 3.75-3.72(4 \mathrm{H}, \mathrm{m}), 3.62-3.58(4 \mathrm{H}, \mathrm{m})$.

Step 3: 4-(4-(2-Benzylpyrrolidin-1-yl)-6-((4-methoxybenzyl)oxy)pyrimidin-2-yl)morpholine. 2-Benzylpyrrolidine (180 mg, $1.12 \mathrm{mmol})$ was added to a solution of 4-(4-chloro-6-((4methoxybenzyl)oxy)pyrimidin-2-yl)morpholine $(250 \mathrm{mg}, 0.75 \mathrm{mmol})$ and DIPEA (195 $\mu \mathrm{L}$, $1.12 \mathrm{mmol})$ in DMF $(2 \mathrm{~mL})$. The reaction mixture was heated at $90^{\circ} \mathrm{C}$. After $21 \mathrm{~h}$ the reaction mixture was cooled to r.t. and diluted with DCM and water. The layers were separated, and the organic extracts dried (phase separator). The solvents were removed under reduced pressure and the resultant oil purified by silica gel column chromatography (gradient elution, $7-60 \%$ ethyl acetate in iso-hexane) to afford the title compound as a colourless oil $(240 \mathrm{mg}$, 69\%). LCMS (ES $\left.{ }^{+}\right) 461(\mathrm{M}+\mathrm{H})^{+}, \mathrm{RT} 1.96 \min$ (Analytical Method F).

Step 4: (R)-6-(2-Benzylpyrrolidin-1-yl)-2-morpholinopyrimidin-4-ol (30). TFA (2 mL) was added to a solution of 4-(4-(2-benzylpyrrolidin-1-yl)-6-((4-methoxybenzyl)oxy)pyrimidin-2yl)morpholine (240 mg, $0.52 \mathrm{mmol})$ in DCM (2 mL) at r.t. with stirring. After $4 \mathrm{~h}$ the reaction mixture was concentrated under reduced pressure. The crude residue was dissolved in DCM, washed with saturated $\mathrm{NaHCO}_{3}$ aqueous solution, dried (phase separator) and concentrated under reduced pressure. The resultant oil was purified by silica gel column chromatography (gradient elution, 2-20\% methanol in DCM) to afford a yellow foaming oil. The enantiomers 
were separated by SFC. (R)-6-(2-Benzylpyrrolidin-1-yl)-2-morpholinopyrimidin-4-ol was triturated with DCM/iso-hexane to afford the title compound as a beige powder (26 mg, 15\%). LCMS (ES $\left.{ }^{+}\right) 341(\mathrm{M}+\mathrm{H})^{+}$, RT $3.25 \mathrm{~min}$ (Analytical Method E), RT $6.31 \mathrm{~min}$ (Analytical Method SFC1, LUX CELLULOSE-4 35\% MeOH; 0.1\% DEA/CO $\left.\mathrm{CO}_{2}\right) ;{ }^{1} \mathrm{H}$ NMR (400 MHz, $\left.\mathrm{CDCl}_{3}\right): \delta 11.26(1 \mathrm{H}, \mathrm{s}), 7.31-7.13(5 \mathrm{H}, \mathrm{m}), 4.85$ - $4.78(1 \mathrm{H}, \mathrm{m}), 4.49-4.48(1 \mathrm{H}, \mathrm{m}), 3.81$ $3.69(9 \mathrm{H}, \mathrm{m}), 3.29-3.22(1 \mathrm{H}, \mathrm{m}), 3.15-3.09(1 \mathrm{H}, \mathrm{m}), 2.68-2.62(1 \mathrm{H}, \mathrm{m}), 1.83-1.77(4 \mathrm{H}$, m). HRMS $\left(\mathrm{ESI}^{+}\right)(\mathrm{M}+\mathrm{H})^{+}$: calcd for $\mathrm{C}_{19} \mathrm{H}_{25} \mathrm{~N}_{4} \mathrm{O}_{2}, 341.1977$; found, 341.2.

(R)-2-(2-Benzylpyrrolidin-1-yl)-6-morpholinopyrimidin-4-ol (31). $\quad$ Step $\quad 1$ : 4-(2-(2Benzylpyrrolidin-1-yl)-6-((4-methoxybenzyl)oxy)pyrimidin-4-yl)morpholine.

Benzylpyrrolidine (180 mg, $1.12 \mathrm{mmol})$ was added to a solution of 4-(2-chloro-6-((4methoxybenzyl)oxy)pyrimidin-4-yl)morpholine (obtained from Step 2 for compound 30) (250 $\mathrm{mg}, 0.75 \mathrm{mmol})$ and DIPEA $(195 \mu \mathrm{L}, 1.12 \mathrm{mmol})$ in DMF $(2 \mathrm{~mL})$. The reaction mixture was heated at $90{ }^{\circ} \mathrm{C}$. After $21 \mathrm{~h}$ the reaction mixture was cooled to r.t. and diluted with DCM and water. The layers were separated, and the organic extracts dried (phase separator). The crude mixture was purified by silica gel column chromatography (gradient elution, 7-60\% EtOAc/iso-hexane) to afford the title compound. LCMS $\left(\mathrm{ES}^{+}\right) 461(\mathrm{M}+\mathrm{H})^{+}$, RT $1.9 \mathrm{~min}$ (Analytical Method F); ${ }^{1} \mathrm{H}$ NMR (400 MHz, $\left.\mathrm{CDCl}_{3}\right): \delta$ 7.40-7.16 (7H, m), 6.89-6.85 (2H, m), 5.35-5.28 (3H, m), 4.40-4.34 (1H, m), 3.80-3.69 (7H, m), 3.63-3.49 $(6 \mathrm{H}, \mathrm{m}), 3.38-3.32(1 \mathrm{H}$, m), $2.57(1 \mathrm{H}, \mathrm{dd}, \mathrm{J}=8.7,12.8 \mathrm{~Hz}), 1.88-1.76(4 \mathrm{H}, \mathrm{m})$.

Step 2: (S)-2-(2-Benzylpyrrolidin-1-yl)-6-morpholinopyrimidin-4-ol and (R)-2-(2benzylpyrrolidin-1-yl)-6-morpholinopyrimidin-4-ol (31). TFA (1.5 $\mathrm{mL})$ was added to a solution of 4-(2-(2-benzylpyrrolidin-1-yl)-6-((4-methoxybenzyl)oxy)pyrimidin-4yl)morpholine $(187 \mathrm{mg}, 0.41 \mathrm{mmol})$ in DCM $(1.5 \mathrm{~mL})$ at r.t. with stirring. After $4 \mathrm{~h}$ the reaction mixture was concentrated under reduced pressure. The crude residue was dissolved in DCM, 
washed with saturated $\mathrm{NaHCO}_{3}$ aqueous solution, dried (phase separator) and concentrated under reduced pressure. The resultant oil was purified by silica gel column chromatography (gradient elution, 2-20\% methanol in DCM) to afford the product. The enantiomers were separated by SFC followed by preparative HPLC to afford the title compounds which were freeze dried from $\mathrm{MeCN} /$ water.

(R)-2-(2-Benzylpyrrolidin-1-yl)-6-morpholinopyrimidin-4-ol (31); LCMS (ES $\left.{ }^{+}\right) 341(\mathrm{M}+\mathrm{H})^{+}$, RT 3.12 min (Analytical Method A), RT 1.37 min (Analytical Method SFC4, LMC CELLULOSE-3 20\% MeOH; 0.1\% DEAISO/CO 2 ); ${ }^{1} \mathrm{H}$ NMR (400 MHz, DMSO-d $\left.{ }_{6}\right): \delta 10.23$ $(1 \mathrm{H}, \mathrm{s}), 7.38$ - $7.33(2 \mathrm{H}, \mathrm{m}), 7.29-7.23(3 \mathrm{H}, \mathrm{m}), 4.85(1 \mathrm{H}, \mathrm{s}), 4.41$ - $4.35(1 \mathrm{H}, \mathrm{m}), 3.70(4 \mathrm{H}$, m, $), 3.50(5 \mathrm{H}, \mathrm{dd}, \mathrm{J}=3.7,3.7 \mathrm{~Hz}), 3.33-3.30(1 \mathrm{H}, \mathrm{m}), 3.17-3.11(1 \mathrm{H}, \mathrm{m}), 2.67-2.59(1 \mathrm{H}$, m), $1.90-1.71(4 \mathrm{H}, \mathrm{m})$. HRMS $\left(\mathrm{ESI}^{+}\right)(\mathrm{M}+\mathrm{H})^{+}$: calcd for $\mathrm{C}_{19} \mathrm{H}_{25} \mathrm{~N}_{4} \mathrm{O}_{2}, 341.1977$; found, 341.1996.

Data is given for the $(S)$-isomer as comparison:

(S)-2-(2-Benzylpyrrolidin-1-yl)-6-morpholinopyrimidin-4-ol; LCMS (ES $\left.{ }^{+}\right) 341(\mathrm{M}+\mathrm{H})^{+}, \mathrm{RT}$ 3.12 min (Analytical Method A), RT 0.94 min (Analytical Method SFC4, LMC CELLULOSE3 20\% MeOH; 0.1\% DEAISO/CO $\left.\mathrm{CO}_{2}\right) ;{ }^{1} \mathrm{H}$ NMR (400 MHz, DMSO-d 6 ): $\delta 10.25(1 \mathrm{H}, \mathrm{s}), 7.36$ $(2 \mathrm{H}, \mathrm{dd}, \mathrm{J}=7.5,7.5 \mathrm{~Hz}), 7.29-7.23(3 \mathrm{H}, \mathrm{m}), 4.86(1 \mathrm{H}, \mathrm{s}), 4.40$ - $4.33(1 \mathrm{H}, \mathrm{m}), 3.72$ - $3.67(4 \mathrm{H}$, m), 3.53 - $3.48(5 \mathrm{H}, \mathrm{m}), 3.34-3.30(1 \mathrm{H}, \mathrm{m}), 3.17-3.10(1 \mathrm{H}, \mathrm{m}), 2.68-2.60(1 \mathrm{H}, \mathrm{m}), 1.90$ $1.69(4 \mathrm{H}, \mathrm{m})$.

(R)-2-(2-Benzylazepan-1-yl)-6-morpholinopyrimidin-4(3H)-one (32). Step 1: (R)-4-(2-(2Benzylazepan-1-yl)-6-((4-methoxybenzyl)oxy)pyrimidin-4-yl)morpholine. 2-(R)Benzylazepane (169 mg, $0.89 \mathrm{mmol})$ was added to a solution of 4-(2-chloro-6-((4methoxybenzyl)oxy)pyrimidin-4-yl)morpholine (obtained from Step 2 for compound 30) (200 $\mathrm{mg}, 0.60 \mathrm{mmol})$ and DIPEA $(156 \mu \mathrm{L}, 0.89 \mathrm{mmol})$ in DMF $(1.6 \mathrm{~mL})$. The reaction mixture was 
heated at $100{ }^{\circ} \mathrm{C}$. After $66 \mathrm{~h}$ the reaction mixture was cooled to r.t. and diluted with DCM and water. The layers were separated, and the organic extracts dried (phase separator). The crude mixture was purified by silica gel column chromatography (gradient elution, 10-80\% EtOAc/iso-hexane) to afford the title compound as a yellow oil (144 mg). ${ }^{1} \mathrm{H}$ NMR (400 MHz, $\left.\mathrm{CDCl}_{3}\right): \delta 7.35(2 \mathrm{H}, \mathrm{d}, \mathrm{J}=9.3 \mathrm{~Hz}), 7.28-7.35(5 \mathrm{H}, \mathrm{m}), 6.88(2 \mathrm{H}, \mathrm{d}, \mathrm{J}=9.3 \mathrm{~Hz}), 5.37-5.23(3 \mathrm{H}$, m), 4.83-4.66 (1H, m), 4.29-4.18 (1H, m), 3.83-3.71 (8H, m), 3.55-3.44 (4H, m), 3.02-2.76 (2H, m), 2.69-2.57 (1H, m), 1.96-1.86 (1H, m), 1.78-1.11 (6H, m).

Step 2: (R)-2-(2-Benzylazepan-1-yl)-6-morpholinopyrimidin-4(3H)-one (32). TFA (1 mL) was added to a solution of $(R)-4-(2-(2-$ benzylazepan-1-yl)-6-((4-methoxybenzyl)oxy)pyrimidin-4yl)morpholine (144 mg, $0.295 \mathrm{mmol})$ in DCM (1 mL) at r.t. with stirring. After $5 \mathrm{~h}$ the reaction mixture was concentrated under reduced pressure. The crude residue was dissolved in DCM, washed with saturated $\mathrm{NaHCO}_{3}$ aqueous solution, dried (phase separator) and concentrated under reduced pressure. The resultant oil was dissolved in DMSO and purified by reverse phase preparative HPLC. The resultant solid was dissolved in DCM and washed with saturated aqueous solution of $\mathrm{NaHCO}_{3}$. The organic extracts were dried (phase separator) and concentrated under reduced pressure followed by freeze drying from $\mathrm{MeCN}$ :water to afford the title compound. LCMS (ES $) 369(\mathrm{M}+\mathrm{H})^{+}$, RT $3.26 \mathrm{~min}$ (Analytical Method A), ${ }^{1} \mathrm{H}$ NMR (400 $\left.\mathrm{MHz}, \mathrm{CDCl}_{3}\right): \delta 7.25-7.19(3 \mathrm{H}, \mathrm{m}), 7.16-7.11(2 \mathrm{H}, \mathrm{m}), 4.90(1 \mathrm{H}, \mathrm{s}), 4.71(1 \mathrm{H}, \mathrm{br} \mathrm{s}), 3.75-$ $3.60(5 \mathrm{H}, \mathrm{m}), 3.53-3.47(4 \mathrm{H}, \mathrm{m}), 2.92-2.82(2 \mathrm{H}, \mathrm{m}), 2.78-2.70(1 \mathrm{H}, \mathrm{m}), 2.08-1.98(1 \mathrm{H}$, m), 1.82 - $1.75(3 \mathrm{H}, \mathrm{m}), 1.49-1.39(2 \mathrm{H}, \mathrm{m}), 1.28-1.12(2 \mathrm{H}, \mathrm{m}), \mathrm{NH}$ not observed. HRMS $\left(\mathrm{ESI}^{+}\right)(\mathrm{M}+\mathrm{H})^{+}$: calcd for $\mathrm{C}_{21} \mathrm{H}_{29} \mathrm{~N}_{4} \mathrm{O}_{2}, 369.229$; found, 369.2317.

4-(7-Methoxy-9H-thioxanthen-4-yl)-6-morpholinopyrimidin-2-ol (34). Step 1: 4-(6-Chloro-2((4-methoxybenzyl)oxy)pyrimidin-4-yl)morpholine (Intermediate 29). Morpholine (4.35g, 0.05 mol) was added to a stirred suspension of 4,6-dichloro-2-((4-methoxybenzyl)oxy)pyrimidine 
and, 2,4-dichloro-6-((4-methoxybenzyl)oxy)pyrimidine (from step 1 of compound 30, 12.8g, $0.045 \mathrm{~mol})$ and cesium carbonate $(32.6 \mathrm{~g}, 0.05 \mathrm{~mol})$ in acetonitrile $(150 \mathrm{~mL})$ at r.t. The resulting suspension was stirred at r.t. for $20 \mathrm{~h}$. The reaction was evaporated, and the residue was treated with DCM $(250 \mathrm{~mL})$ and water $(50 \mathrm{~mL})$. The DCM was separated and evaporated to give a colorless oil which was purified by flash chromatography ( $0 \%$ to $20 \%$ ethyl acetate in isohexane) to give $5.74 \mathrm{~g}\left(34 \%\right.$ over two steps) of the title compound as a colorless solid. ${ }^{1} \mathrm{H}$ NMR (400 MHz, $\left.\mathrm{CDCl}_{3}\right): \delta$ 7.39-7.35 (2H, m), 6.89-6.86 (2H, m), $6.17(1 \mathrm{H}, \mathrm{s}), 5.28(2 \mathrm{H}, \mathrm{s})$, $3.73(3 \mathrm{H}, \mathrm{s}), 3.75-3.72(4 \mathrm{H}, \mathrm{m}), 3.62-3.58(4 \mathrm{H}, \mathrm{m})$.

Step 2: 4-(6-(7-Methoxy-9H-thioxanthen-4-yl)-2-((4-methoxybenzyl)oxy)pyrimidin-4yl)morpholine (Intermediate 33). 4-(6-Chloro-2-((4-methoxybenzyl)oxy)pyrimidin-4yl)morpholine (0.2 g, $0.6 \mathrm{mmol})$ was stirred with 2-(7-methoxy- $9 H$-thioxanthen-4-yl)-4,4,5,5tetramethyl-1,3,2-dioxaborolane ${ }^{9}(0.21 \mathrm{~g}, 0.6 \mathrm{mmol})$, potassium carbonate $(0.21 \mathrm{~g}, 1.5 \mathrm{mmol})$, and $\mathrm{Pd}\left(\mathrm{PPh}_{3}\right)_{4}$ in dioxane $(20 \mathrm{~mL}$, dry) under nitrogen for $15 \mathrm{~min}$. The mixture was then stirred at $70{ }^{\circ} \mathrm{C}$ for $20 \mathrm{~h}$. The reaction was evaporated, and the residue treated with DCM $(50 \mathrm{~mL})$ and water $(25 \mathrm{~mL})$, the DCM was separated and evaporated to give a yellow oil used in the next step without further purification.

Step 3: 4-(7-Methoxy-9H-thioxanthen-4-yl)-6-morpholinopyrimidin-2-ol (34). The crude 4-(6(7-methoxy-9H-thioxanthen-4-yl)-2-((4-methoxybenzyl)oxy)pyrimidin-4-yl)morpholine from Step 3 was dissolved in DCM $(2.5 \mathrm{~mL})$ and TFA $(2.5 \mathrm{~mL})$ was added, after standing for $1 \mathrm{~h}$ the solution was evaporated to give a yellow oil. The oil was dissolved in DCM (10 mL) and washed with saturated $\mathrm{NaHCO}_{3}(10 \mathrm{~mL})$, the DCM was separated and evaporated to give a colorless oil which was dissolved in a small volume of acetonitrile and loaded onto a $20 \mathrm{~g} \mathrm{C18}$ cartridge, eluting with acetonitrile in water (10\% to 50\%) to give $43 \mathrm{mg}$ ( $14 \%$ over two steps) of the title compound as a colorless solid. $\mathrm{LCMS}_{\left(\mathrm{ES}^{+}\right)} 408(\mathrm{M}+\mathrm{H})^{+}$, RT $2.93 \mathrm{~min}($ Analytical Method A); ${ }^{1} \mathrm{H}$ NMR (400 MHz, DMSO-d 6$) 10.95(1 \mathrm{H}, \mathrm{s}), 7.60$ - $7.57(1 \mathrm{H}, \mathrm{m}), 7.45$ - $7.34(3 \mathrm{H}$, 
m), $7.14(1 \mathrm{H}, \mathrm{d}, \mathrm{J}=2.8 \mathrm{~Hz}), 6.87(1 \mathrm{H}, \mathrm{dd}, \mathrm{J}=2.8,8.6 \mathrm{~Hz}), 6.10(1 \mathrm{H}, \mathrm{s}), 3.96(2 \mathrm{H}, \mathrm{s}), 3.81(3 \mathrm{H}$, s), 3.75-3.65 (8H, m). HRMS $\left(\mathrm{ESI}^{+}\right)(\mathrm{M}+\mathrm{H})^{+}$: calcd for $\mathrm{C}_{22} \mathrm{H}_{22} \mathrm{~N}_{3} \mathrm{O}_{3} \mathrm{~S}$, 408.1382; found, 408.1388.

4-[2-Methoxy-6-(7-methoxy-9H-thioxanthen-4-yl)-4-pyridyl]morpholine (40). Step 1: 4-(2,6Dichloro-4-pyridyl)morpholine (Intermediate 37). A mixture of 2,6-dichloro-4-iodopyridine $(1 \mathrm{~g}, 3.7 \mathrm{mmol})$ and $\mathrm{NaO} t \mathrm{Bu}(711 \mathrm{mg}, 7.4 \mathrm{mmol})$ in dry toluene $(2 \mathrm{~mL})$ was sparged with $\mathrm{N}_{2}$ for $20 \mathrm{~min}$. [1,1'-Bis(di-tert-butylphosphino)ferrocene]dichloropalladium(II) (60 mg, 0.093 mmol $)$ and morpholine $(320 \mu \mathrm{L}, 3.7 \mathrm{mmol})$ were then added, the reaction tube was sealed under $\mathrm{N}_{2}$ and stirred at $50{ }^{\circ} \mathrm{C}$ for 23 h. After cooling to r.t., the mixture was filtered through Celite, washing thoroughly with $\mathrm{CH}_{2} \mathrm{Cl}_{2}$ and the filtrate was evaporated. Purification by silica gel chromatography (gradient elution, 0-30\% EtOAc/iso-hexane) gave $415 \mathrm{mg}$ (48\%) of the title compound. LCMS (ES $\left.{ }^{+}\right) 232(\mathrm{M}+\mathrm{H})^{+}$, RT 2.58 min (Analytical Method F); ${ }^{1} \mathrm{H}$ NMR (400 $\left.\mathrm{MHz}, \mathrm{CDCl}_{3}\right): \delta 6.59(2 \mathrm{H}, \mathrm{s}), 3.84-3.80(4 \mathrm{H}, \mathrm{m}), 3.33$ - $3.29(4 \mathrm{H}, \mathrm{m})$.

Step 2: 4-(2-Chloro-6-methoxy-4-pyridyl)morpholine (Intermediate 39). 4-(2,6-Dichloro-4pyridyl)morpholine $(2.28 \mathrm{~g}, 9.78 \mathrm{mmol})$ was suspended in sodium methoxide in methanol (18 $\mathrm{mL}$ of $30 \mathrm{wt} \%$ solution) diluted with methanol $(5 \mathrm{~mL})$. The mixture was heated to $70{ }^{\circ} \mathrm{C}$ for $89 \mathrm{~h}$ after which the solvent was evaporated and partitioned between EtOAc and water. The aqueous phase was then re-extracted with EtOAc (x2), the combined organic phases were dried $\left(\mathrm{MgSO}_{4}\right)$ and evaporated. Purification by silica gel chromatography (gradient elution, $0-10 \%$ $\left.\mathrm{Et}_{2} \mathrm{O} / \mathrm{CH}_{2} \mathrm{Cl}_{2}\right)$ afforded $1.80 \mathrm{~g}(81 \%)$ of the title compound. LCMS $\left(\mathrm{ES}^{+}\right) 354(\mathrm{M}+\mathrm{H})^{+}, \mathrm{RT} 3.53$ min (Analytical Method F).

Step 3: 4-[2-Methoxy-6-(7-methoxy-9H-thioxanthen-4-yl)-4-pyridyl]morpholine (40). Following Method K from 4-(2-chloro-6-methoxy-4-pyridyl)morpholine (32 mg, 0.14 mmol) and 2-(7-methoxy-9H-thioxanthen-4-yl)-4,4,5,5-tetramethyl-1,3,2-dioxaborolane ${ }^{9} \quad(50 \mathrm{mg}$, $0.14 \mathrm{mmol}$ ) to obtain the crude material. Purification by silica gel chromatography (gradient 
elution, 0-25\% EtOAc/iso-hexane) followed by SFC purification and freeze drying from acetonitrile-water gave $23 \mathrm{mg}(39 \%)$ of the title compound. LCMS $\left(\mathrm{ES}^{+}\right) 421(\mathrm{M}+\mathrm{H})^{+}, \mathrm{RT}$ $3.68 \mathrm{~min}$ (Analytical Method B); ${ }^{1} \mathrm{H}$ NMR (400 MHz, DMSO-d 6 ): $\delta 7.56-7.48(2 \mathrm{H}, \mathrm{m}), 7.38$ - $7.30(2 \mathrm{H}, \mathrm{m}), 7.11(1 \mathrm{H}, \mathrm{d}, \mathrm{J}=2.5 \mathrm{~Hz}), 6.85(1 \mathrm{H}, \mathrm{d}, \mathrm{J}=2.0 \mathrm{~Hz}), 6.80(1 \mathrm{H}, \mathrm{dd}, \mathrm{J}=2.7,8.5 \mathrm{~Hz})$, $6.25(1 \mathrm{H}, \mathrm{d}, \mathrm{J}=2.0 \mathrm{~Hz}), 3.95(5 \mathrm{H}, \mathrm{d}, \mathrm{J}=6.8 \mathrm{~Hz}), 3.80(3 \mathrm{H}, \mathrm{s}), 3.79-3.75(4 \mathrm{H}, \mathrm{m}), 3.40$ - 3.38 (4H, m). HRMS $\left(\mathrm{ESI}^{+}\right)(\mathrm{M}+\mathrm{H})^{+}$: calcd for $\mathrm{C}_{24} \mathrm{H}_{25} \mathrm{~N}_{2} \mathrm{O}_{3} \mathrm{~S}, 421.1586$; found, 421.1591 .

6-(2-Benzylpyrrolidin-1-yl)-4-morpholinopyridin-2(1H)-one $\quad$ (43). $\quad$ Step $\quad$ 1: $\quad$ 4-(2,6Dichloropyridin-4-yl)morpholine (Intermediate 37). 2,6-Dichloropyridin-4-amine (10 g, 61.3 mmol) was dissolved in DMF $(200 \mathrm{~mL})$ and cooled to $0{ }^{\circ} \mathrm{C}$. Sodium hydride $(6.13 \mathrm{~g}, 153$ mmol) was added portionwise over $20 \mathrm{~min}$. After $15 \mathrm{~min}$ 1-chloro-2-(2-chloroethoxy)ethane $(8.63 \mathrm{~mL}, 73.6 \mathrm{mmol})$ was added dropwise. The reaction mixture was warmed to r.t. and stirred for $21.5 \mathrm{~h}$. After this time the reaction was cooled to $0{ }^{\circ} \mathrm{C}$ and cautiously quenched with water. The DMF was removed under reduced pressure. The resultant residue was dissolved in DCM and washed with water. The organic layers were dried $\left(\mathrm{MgSO}_{4}\right)$, filtered and concentrated under reduced pressure. The crude product was triturated with EtOAc and iso-hexane to afford the title compound as a white solid (9.27 g, 65\%). LCMS (ES+) 233, $235(\mathrm{M}+\mathrm{H})+$, RT 2.67 min (Analytical Method F); ${ }^{1} \mathrm{H}$ NMR (400 MHz, $\left.\mathrm{CDCl}_{3}\right): \delta 6.59(2 \mathrm{H}, \mathrm{s}), 3.84-3.80(4 \mathrm{H}, \mathrm{m})$, 3.33-3.29 (4H, m).

Step 2: 4-(2-Chloro-6-((4-methoxybenzyl)oxy)pyridin-4-yl)morpholine (Intermediate 41). Following Method A using 4-(2,6-dichloropyridin-4-yl)morpholine (9.27 g, 39.8 mmol). The crude product was triturated with EtOAc and iso-hexane to afford the title compound as an offwhite solid (11.4 g, 86\%). ${ }^{1} \mathrm{H}$ NMR (400 MHz, $\left.\mathrm{CDCl}_{3}\right): \delta 7.39$ - $7.35(2 \mathrm{H}, \mathrm{m}), 6.91-6.88(2 \mathrm{H}$, m), $6.40(1 \mathrm{H}, \mathrm{d}, \mathrm{J}=2.0 \mathrm{~Hz}), 5.99(1 \mathrm{H}, \mathrm{d}, \mathrm{J}=2.0 \mathrm{~Hz}), 5.25(2 \mathrm{H}, \mathrm{s}), 3.81-3.77(7 \mathrm{H}, \mathrm{m}), 3.25$ $3.21(4 \mathrm{H}, \mathrm{m})$. 
Step 3: 4-(2-(2-Benzylpyrrolidin-1-yl)-6-((4-methoxybenzyl)oxy)pyridin-4-yl)morpholine.

Following Method C, starting from 2-benzylpyrrolidine (53 mg, $0.30 \mathrm{mmol}$ ) and 4-(2-chloro6-((4-methoxybenzyl)oxy)pyridin-4-yl)morpholine $(101 \mathrm{mg}, 0.30 \mathrm{mmol})$. After heating at $105^{\circ} \mathrm{C}$ for $18.5 \mathrm{~h}$, a further 0.5 eq pyrrolidine, 1.6 eq cesium carbonate, $0.025 \mathrm{eq}, \operatorname{Pd}_{2}(\mathrm{dba})_{3}$, and 0.1 eq BINAP were added and the reaction heated at $105^{\circ} \mathrm{C}$ for a further $23 \mathrm{~h}$. The reaction was cooled to r.t. and filtered through Celite, washing with DCM and the reaction concentrated under reduced pressure. The reaction was repeated on the same scale with 1,4-dioxane as the solvent. The crude mixtures of both reactions were combined and purified by silica gel column chromatography (gradient elution, 0-25\% EtOAc/iso-hexane) to afford the title compound (170 mg, 61\%). LCMS (ES $\left.{ }^{+}\right) 460(\mathrm{M}+\mathrm{H})^{+}$, RT $2.57 \min \left(\right.$ Analytical Method F); ${ }^{1} \mathrm{H}$ NMR $(400 \mathrm{MHz}$, $\left.\mathrm{CDCl}_{3}\right): \delta$ 7.40-7.35 (2H, m), 7.29-7.17 (5H, m), 6.90-6.86 (2H, m), $5.62(1 \mathrm{H}, \mathrm{d}, \mathrm{J}=1.8 \mathrm{~Hz})$, 5.43-5.27 (3H, m), 4.43-4.36 (1H, m), 3.84-3.76 (7H, m), 3.44-3.38 (1H, m), 3.31-3.17 (5H, m), $2.62(1 \mathrm{H}, \mathrm{dd}, \mathrm{J}=9.3,13.2 \mathrm{~Hz}), 1.93-1.78(3 \mathrm{H}, \mathrm{m}), 1.58-1.24(2 \mathrm{H}, \mathrm{m})$.

Step 4: 6-(2-Benzylpyrrolidin-1-yl)-4-morpholinopyridin-2(1H)-one (43). Following Method E from 4-(2-(2-benzylpyrrolidin-1-yl)-6-((4-methoxybenzyl)oxy)pyridin-4-yl)morpholine (150 mg, $0.32 \mathrm{mmol}$ ). Purification by reverse phase preparative HPLC yielded the title compound. LCMS $\left(\mathrm{ES}^{+}\right) 340(\mathrm{M}+\mathrm{H})^{+}$, RT 2.96 min (Analytical Method B); ${ }^{1} \mathrm{H}$ NMR (400 MHz, DMSO-d 6 ): $\delta 9.68(1 \mathrm{H}, \mathrm{s}), 7.32$ - $7.29(4 \mathrm{H}, \mathrm{m}), 7.25-7.19(1 \mathrm{H}, \mathrm{m}), 5.15(2 \mathrm{H}, \mathrm{s}), 4.22$ $4.19(1 \mathrm{H}, \mathrm{m}), 3.71-3.67(4 \mathrm{H}, \mathrm{m}), 3.42-3.36(1 \mathrm{H}, \mathrm{m}), 3.21-3.14(5 \mathrm{H}, \mathrm{m}), 2.97(1 \mathrm{H}, \mathrm{dd}, \mathrm{J}=3.5$,

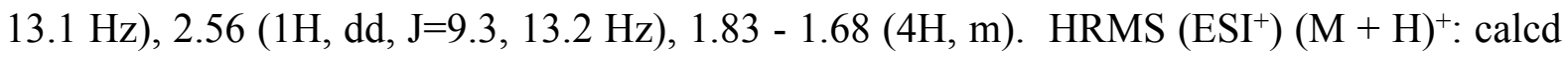
for $\mathrm{C}_{20} \mathrm{H}_{26} \mathrm{~N}_{3} \mathrm{O}_{2}, 340.2025$; found, 340.2029 .

(R)-6-(2-Benzylpyrrolidin-1-yl)-4-morpholinopyridin-2(1H)-one

(44) and (S)-6-(2benzylpyrrolidin-1-yl)-4-morpholinopyridin-2(1H)-one (45). A racemic sample of compound 43 was separated into its constituent enantiomers using chiral SFC. 
6-[(2R)-2-Benzylpyrrolidin-1-yl]-4-morpholino-1H-pyridin-2-one (44); LCMS (ES $\left.{ }^{+}\right) 340$ $(\mathrm{M}+\mathrm{H})^{+}$, RT 2.97 min (Analytical Method B); RT 4.62 min (Analytical Method SFC1, YMC CELLULOSE-SC, 45/55 IPA (0.1\% DEA)/CO 2$) ;{ }^{1} \mathrm{H}$ NMR (400 MHz, DMSO-d 6 ): $\delta 9.68(1 \mathrm{H}$, s), 7.32 - $7.29(4 \mathrm{H}, \mathrm{m}), 7.25$ - $7.19(1 \mathrm{H}, \mathrm{m}), 5.15(2 \mathrm{H}, \mathrm{s}), 4.22$ - $4.19(1 \mathrm{H}, \mathrm{m}), 3.71-3.67(4 \mathrm{H}$, m), $3.42-3.36(1 \mathrm{H}, \mathrm{m}), 3.21-3.14(5 \mathrm{H}, \mathrm{m}), 2.97(1 \mathrm{H}, \mathrm{dd}, \mathrm{J}=3.5,13.1 \mathrm{~Hz}), 2.56(1 \mathrm{H}, \mathrm{dd}, \mathrm{J}=9.3$, 13.2 Hz), $1.83-1.68(4 \mathrm{H}, \mathrm{m})$. HRMS $\left(\mathrm{ESI}^{+}\right)(\mathrm{M}+\mathrm{H})^{+}$: calcd for $\mathrm{C}_{20} \mathrm{H}_{26} \mathrm{~N}_{3} \mathrm{O}_{2}, 340.2025$; found, 340.2032 .

6-[(2S)-2-Benzylpyrrolidin-1-yl]-4-morpholino-1H-pyridin-2-one (45); LCMS (ES $\left.{ }^{+}\right) 340$ $(\mathrm{M}+\mathrm{H})^{+}$, RT $2.96 \mathrm{~min}$ (Analytical Method B); RT $5.78 \mathrm{~min}$ (Analytical Method SFC1, YMC CELLULOSE-SC, 45/55 IPA (0.1\% DEA)/CO 2$) ;{ }^{1} \mathrm{H}$ NMR (400 MHz, DMSO-d 6 ): $\delta 9.68(1 \mathrm{H}$, s), $7.32-7.29(4 \mathrm{H}, \mathrm{m}), 7.25-7.19(1 \mathrm{H}, \mathrm{m}), 5.15(2 \mathrm{H}, \mathrm{s}), 4.22-4.19(1 \mathrm{H}, \mathrm{m}), 3.71-3.67(4 \mathrm{H}$, m), $3.42-3.36(1 \mathrm{H}, \mathrm{m}), 3.21-3.14(5 \mathrm{H}, \mathrm{m}), 2.97(1 \mathrm{H}, \mathrm{dd}, \mathrm{J}=3.5,13.1 \mathrm{~Hz}), 2.56(1 \mathrm{H}, \mathrm{dd}, \mathrm{J}=9.3$, $13.1 \mathrm{~Hz}), 1.83-1.68(4 \mathrm{H}, \mathrm{m})$.

Absolute synthesis of compound 45. Step 1: Tert-butyl (S)-2(hydroxy(phenyl)methyl)pyrrolidine-1-carboxylate. Phenylmagnesium bromide $(15 \mathrm{~mL}, 15$ mmol, 1.0 M in THF) was added dropwise to a solution of tert-butyl (2S)-2-formylpyrrolidine1-carboxylate $(2.00 \mathrm{~g}, 10 \mathrm{mmol})$ in dry THF $(20 \mathrm{~mL})$ at $-78^{\circ} \mathrm{C}$. The reaction mixture was stirred at $-78{ }^{\circ} \mathrm{C}$ for $2.5 \mathrm{~h}$, before quenching with saturated aqueous $\mathrm{NH}_{4} \mathrm{Cl}$ solution and diluted with water $(10 \mathrm{~mL})$. The mixture was extracted with EtOAc (x 2), dried $\left(\mathrm{MgSO}_{4}\right)$, filtered and concentrated under reduced pressure. Purification by silica gel column chromatography (gradient elution, 0-25\% EtOAc/iso-hexane) gave the title compound as a complex mixture of diastereomers and rotamers: ${ }^{1} \mathrm{H} \mathrm{NMR}\left(400 \mathrm{MHz}, \mathrm{CDCl}_{3}\right): \delta 7.39-7.28(5 \mathrm{H}, \mathrm{m}), 5.91-5.37(1 \mathrm{H}$, m), 5.20-4.82 (1H, m), 4.58-4.26 (1H, m), 3.59-3.24 (2H, m), 2.07-1.65 (4H, m), 1.55-1.44 $(9 \mathrm{H}, \mathrm{m})$. 
Step 2: Tert-butyl (S)-2-(((1H-imidazole-1-carbonothioyl)oxy)(phenyl)methyl)pyrrolidine-1carboxylate. To a solution of tert-butyl (S)-2-(hydroxy(phenyl)methyl)pyrrolidine-1carboxylate $(242 \mathrm{mg}, 0.87 \mathrm{mmol})$ in anhydrous THF $(10 \mathrm{~mL})$ was added DMAP $(11 \mathrm{mg}, 0.087$ mmol) and diimidazole thiocarbonyl $(233 \mathrm{mg}, 1.31 \mathrm{mmol})$. The resultant solution was heated at reflux overnight. The solution was cooled to r.t. and the solvents removed in vacuo. The residue was dissolved in DCM, washed with water and the layers separated and dried (phase separator). The DCM was removed in vacuo and the resultant residue was purified by silica gel column chromatography (gradient 0-50\% EtOAc/iso-hexane) to give the title compound. ${ }^{1} \mathrm{H}^{1} \mathrm{NMR}\left(400 \mathrm{MHz}, \mathrm{CDCl}_{3}\right): \delta 8.52-8.39(1 \mathrm{H}, \mathrm{m}), 7.81-7.67(1 \mathrm{H}, \mathrm{m}), 7.48-7.28(6 \mathrm{H}, \mathrm{m}), 5.25$ $(1 \mathrm{H}, \mathrm{d}, \mathrm{J}=4.0 \mathrm{~Hz}), 4.19-4.10(1 \mathrm{H}, \mathrm{m}), 3.85-3.67(2 \mathrm{H}, \mathrm{m}), 2.23-2.09(2 \mathrm{H}, \mathrm{m}), 1.74-1.64(2 \mathrm{H}$, $\mathrm{m}), 1.43-1.35(9 \mathrm{H}, \mathrm{m})$.

Step 3: Tert-butyl (S)-2-benzylpyrrolidine-1-carboxylate. To a solution of tert-butyl (S)-2(((1H-imidazole-1-carbonothioyl)oxy)(phenyl)methyl)pyrrolidine-1-carboxylate (195 mg, 0.5 mmol) in anhydrous toluene $(2 \mathrm{~mL})$ was added tributyltin hydride $(0.41 \mathrm{~mL}, 1.51 \mathrm{mmol})$ and AIBN (16 mg, $0.1 \mathrm{mmol})$. The solution was heated at reflux for $6 \mathrm{~h}$ and then cooled to r.t. The solvent was removed in vacuo and the residue purified by silica gel column chromatography (gradient $0-20 \%$ EtOAc/iso-hexane) to give the title compound as a mixture of rotamers. ${ }^{1} \mathrm{H}$ NMR (400 MHz, $\left.\mathrm{CDCl}_{3}\right): \delta$ 7.31-7.13 (5H, m), 4.14-3.89 (1H, m), 3.44-3.02 (3H, m), 2.61$2.47(1 \mathrm{H}, \mathrm{m}), 1.79-1.48(13 \mathrm{H}, \mathrm{m})$.

Step 4: (S)-2-Benzylpyrrolidine. Tert-butyl (S)-2-benzylpyrrolidine-1-carboxylate (82 mg, 0.31 mmol) was dissolved in $4 \mathrm{~N} \mathrm{HCl} /$ dioxane $(5 \mathrm{ml})$ and stirred at $\mathrm{r} . t$. for $1 \mathrm{~h}$. The solvent was removed in vacuo and the residue was purified by SCX chromatography (eluting with $\mathrm{MeOH} / \mathrm{DCM} 1: 1$ and then collecting with $10 \% 7 \mathrm{~N} \mathrm{NH}_{3}$ in $\mathrm{MeOH} / \mathrm{MeOH}$ ) to give the title 
compound. ${ }^{1} \mathrm{H}$ NMR $\left(400 \mathrm{MHz}, \mathrm{CDCl}_{3}\right): \delta$ 7.32-7.18 $(5 \mathrm{H}, \mathrm{m}), 3.28-3.20(1 \mathrm{H}, \mathrm{m}), 3.07-3.01$ $(1 \mathrm{H}, \mathrm{m}), 2.86-2.71(3 \mathrm{H}, \mathrm{m}), 2.10(1 \mathrm{H}, \mathrm{s}), 1.88-1.62(3 \mathrm{H}, \mathrm{m}), 1.44-1.35(1 \mathrm{H}, \mathrm{m})$.

Step 5: (S)-4-(2-(2-Benzylpyrrolidin-1-yl)-6-((4-methoxybenzyl)oxy)pyridin-4-yl)morpholine. Following Method C starting from $(S)$-2-benzylpyrrolidine (26 mg, $0.16 \mathrm{mmol}$ ) and 4-(2chloro-6-((4-methoxybenzyl)oxy)pyridin-4-yl)morpholine (53 mg, $0.16 \mathrm{mmol}$, Scaffold 1). After heating at $105^{\circ} \mathrm{C}$ for $115 \mathrm{~h}$. The reaction mixture was cooled to r.t., filtered through Celite and concentrated under reduced pressure. The crude mixture was purified by silica gel column chromatography (gradient elution, 0-20\% EtOAc/iso-hexane) to give the title compound. LCMS (ES ${ }^{+} 460(\mathrm{M}+\mathrm{H})^{+}$, RT $1.41 \min$ (Analytical Method F).

Step 6: (S)-6-(2-Benzylpyrrolidin-1-yl)-4-morpholinopyridin-2(1H)-one (45). Following Method E starting from (S)-4-(2-(2-benzylpyrrolidin-1-yl)-6-((4-methoxybenzyl)oxy)pyridin4-yl)morpholine (35 $\mathrm{mg}, 0.08 \mathrm{mmol}$ ). The crude material was purified by reverse phase preparative HPLC and freeze dried from $\mathrm{MeCN} / \mathrm{H}_{2} \mathrm{O}$ to give the title compound. LCMS $\left(\mathrm{ES}^{+}\right)$ $340(\mathrm{M}+\mathrm{H})^{+}$, RT $2.74 \min ($ Analytical Method A); RT 7.62 min (Analytical Method SFC1, YMC CELLULOSE-SC, 45/55 IPA (0.1\% DEA)/CO $\left.\mathrm{CO}_{2}\right) ;{ }^{1} \mathrm{H}$ NMR (400 MHz, DMSO-d $\left.{ }_{6}\right): \delta$ $9.68(1 \mathrm{H}, \mathrm{s}), 7.32$ - $7.29(4 \mathrm{H}, \mathrm{m}), 7.25-7.19(1 \mathrm{H}, \mathrm{m}), 5.15(2 \mathrm{H}, \mathrm{s}), 4.22$ - $4.19(1 \mathrm{H}, \mathrm{m}), 3.71$ $3.67(4 \mathrm{H}, \mathrm{m}), 3.42-3.36(1 \mathrm{H}, \mathrm{m}), 3.21-3.14(5 \mathrm{H}, \mathrm{m}), 2.97(1 \mathrm{H}, \mathrm{dd}, \mathrm{J}=3.5,13.1 \mathrm{~Hz}), 2.56(1 \mathrm{H}$, dd, $\mathrm{J}=9.3,13.1 \mathrm{~Hz}), 1.83-1.68(4 \mathrm{H}, \mathrm{m})$. HRMS $\left(\mathrm{ESI}^{+}\right)(\mathrm{M}+\mathrm{H})^{+}$: calcd for $\mathrm{C}_{20} \mathrm{H}_{26} \mathrm{~N}_{3} \mathrm{O}_{2}$, 340.2025; found, 340.205. The presence of $11 \%$ of the $(R)$-enantiomer in the sample allowed the absolute stereochemistry of the previously prepared samples of compounds $\mathbf{4 4}$ and $\mathbf{4 5}$ to be assigned based on their order of elution under the near identical analysis conditions.

For all subsequent chiral compounds where the enantiomers were separated by Chiral SFC purification, the stereochemical assignments were made based on the biological activity of each enantiomer in the biochemical assay: all $R$-enantiomers were assigned as the bioactive 
conformation based on the biological activity results obtained by the chirally pure synthesized (R)-6-(2-benzylazepan-1-yl)-4-morpholinopyridin-2(1H)-one and the lack of biological activity shown by (S)-6-(2-benzylpyrrolidin-1-yl)-4-morpholinopyridin-2(1H)-one.

The compounds below were made by the same synthetic route as compound $\mathbf{4 3}$, starting from the indicated amine and either 4-(2-chloro-6-((4-methoxybenzyl)oxy)pyridin-4-yl)morpholine or $\quad(R)-4-(2-c h l o r o-6-((4-m e t h o x y b e n z y l) o x y) p y r i d i n-4-y l)-2-m e t h y l m o r p h o l i n e \quad$ as appropriate. Buchwald conditions Method D were used to couple the amine. The isomers were isolated after purification by Chiral SFC.

6-(3-Benzylpyrrolidin-1-yl)-4-morpholinopyridin-2(1H)-one (46). LCMS (ES $\left.{ }^{+}\right) 340(\mathrm{M}+\mathrm{H})^{+}$, RT 2.76 min (Analytical Method A); ${ }^{1} \mathrm{H}$ NMR (400 MHz, $\left.\mathrm{CDCl}_{3}\right)$ 7.33-7.16 (5H, m), $5.17(1 \mathrm{H}$, d, J=2.0 Hz), $4.73(1 \mathrm{H}, \mathrm{d}, \mathrm{J}=2.0 \mathrm{~Hz}), 3.77(4 \mathrm{H}, \mathrm{t}, \mathrm{J}=5.0 \mathrm{~Hz}), 3.49-3.32(3 \mathrm{H}, \mathrm{m}), 3.23(4 \mathrm{H}, \mathrm{t}$, $\mathrm{J}=5 \mathrm{~Hz}), 3.05(1 \mathrm{H}, \mathrm{dd}, \mathrm{J}=8.0,9.0 \mathrm{~Hz}), 2.75(2 \mathrm{H}, \mathrm{m}), 2.61(1 \mathrm{H}, \mathrm{m}), 2.12(1 \mathrm{H}, \mathrm{m}), 1.76(1 \mathrm{H}, \mathrm{m})$. $\mathrm{NH}$ not observed. HRMS $\left(\mathrm{ESI}^{+}\right)(\mathrm{M}+\mathrm{H})^{+}$: calcd for $\mathrm{C}_{20} \mathrm{H}_{26} \mathrm{~N}_{3} \mathrm{O}_{2}, 340.2025$; found, 340.2037. 6-((1S,2S,5R)-2-Benzyl-3-azabicyclo[3.1.0]hexan-3-yl)-4-morpholinopyridin-2(1H)-one (50). LCMS (ES $\left.{ }^{+}\right) 352(\mathrm{M}+\mathrm{H})^{+}$, RT $2.95 \min$ (Analytical Method A); RT 1.58 min (Analytical Method SFC4,YMC AMYLOSE-C, 30/70 MeOH + 0.1\% DEAISO/CO 2$) ;{ }^{1} \mathrm{H}$ NMR (400 MHz, $\left.\mathrm{CDCl}_{3}\right): \delta 7.35-7.24(5 \mathrm{H}, \mathrm{m}), 5.29(1 \mathrm{H}, \mathrm{d}, \mathrm{J}=2.0 \mathrm{~Hz}), 5.02(1 \mathrm{H}, \mathrm{d}, \mathrm{J}=2.0 \mathrm{~Hz}), 4.00-3.94(1 \mathrm{H}$, m), $3.91(1 \mathrm{H}, \mathrm{d}, \mathrm{J}=8.4 \mathrm{~Hz}), 3.82$ - $3.78(4 \mathrm{H}, \mathrm{m}), 3.45(1 \mathrm{H}, \mathrm{dd}, \mathrm{J}=3.8,9.1 \mathrm{~Hz}), 3.30$ - $3.23(5 \mathrm{H}$, m), $2.54(1 \mathrm{H}, \mathrm{dd}, \mathrm{J}=9.9,13.6 \mathrm{~Hz}), 1.71-1.65(2 \mathrm{H}, \mathrm{m}), 0.77(1 \mathrm{H}, \mathrm{dt}, \mathrm{J}=5.1,8.0 \mathrm{~Hz}), 0.62(1 \mathrm{H}$, q, $\mathrm{J}=4.5 \mathrm{~Hz}), \mathrm{NH}$ not observed. HRMS $(\mathrm{ESI}+)(\mathrm{M}+\mathrm{H})+$ : calcd for $\mathrm{C}_{21} \mathrm{H}_{26} \mathrm{~N}_{3} \mathrm{O}_{2}, 352.2025$; found, 352.2041 .

6-((1R,2S, 5S)-2-Benzyl-3-azabicyclo[3.1.0]hexan-3-yl)-4-((R)-2-methylmorpholino) pyridin2(1H)-one (53). LCMS (ES $\left.{ }^{+}\right) 366(\mathrm{M}+\mathrm{H})^{+}$, RT $2.91 \mathrm{~min}$ (Analytical Method A); RT 2.43 min (Analytical Method SFC4,YMC AMYLOSE-C, 30/70 IPA + 0.1\% DEAISO/CO ${ }_{2}$; ${ }^{1} \mathrm{H}$ NMR $\left(400 \mathrm{MHz}, \mathrm{CDCl}_{3}\right): \delta 7.30$ - $7.16(5 \mathrm{H}, \mathrm{m}), 5.22(1 \mathrm{H}, \mathrm{d}, \mathrm{J}=2.0 \mathrm{~Hz}), 4.81(1 \mathrm{H}, \mathrm{d}, \mathrm{J}=2.0 \mathrm{~Hz}), 4.26$ 
$(1 \mathrm{H}, \mathrm{dd}, \mathrm{J}=3.4,8.0 \mathrm{~Hz}), 3.97(1 \mathrm{H}, \mathrm{dd}, \mathrm{J}=2.3,11.6 \mathrm{~Hz}), 3.73-3.62(2 \mathrm{H}, \mathrm{m}), 3.54-3.46(2 \mathrm{H}$, m), $3.35(1 \mathrm{H}, \mathrm{d}, \mathrm{J}=9.1 \mathrm{~Hz}), 3.14(1 \mathrm{H}, \mathrm{dd}, \mathrm{J}=4.0,9.1 \mathrm{~Hz}), 3.02-2.90(2 \mathrm{H}, \mathrm{m}), 2.79(1 \mathrm{H}, \mathrm{dd}$, $\mathrm{J}=8.0,13.0 \mathrm{~Hz}), 2.60(1 \mathrm{H}, \mathrm{dd}, \mathrm{J}=10.4,13.0 \mathrm{~Hz}), 1.55-1.43(2 \mathrm{H}, \mathrm{m}), 1.23(3 \mathrm{H}, \mathrm{d}, \mathrm{J}=6.3 \mathrm{~Hz})$, $0.65(1 \mathrm{H}, \mathrm{dt}, \mathrm{J}=5.1,7.7 \mathrm{~Hz}), 0.20(1 \mathrm{H}, \mathrm{q}, \mathrm{J}=4.4 \mathrm{~Hz}), \mathrm{NH}$ not observed. HRMS $\left(\mathrm{ESI}^{+}\right)(\mathrm{M}+$ $\mathrm{H})^{+}$: calcd for $\mathrm{C}_{22} \mathrm{H}_{28} \mathrm{~N}_{3} \mathrm{O}_{2}, 366.2181$; found, 366.2184 .

(R)-6-(2-Benzylpiperidin-1-yl)-4-morpholinopyridin-2(1H)-one (54). LCMS $\quad\left(\mathrm{ES}^{+}\right) 354$ $(\mathrm{M}+\mathrm{H})^{+}$, RT $2.84 \min$ (Analytical Method A); RT 2.68 min (Analytical Method SFC4, YMC CELLULOSE-C + 0.1\% DEAISO 25\% IPA) ${ }^{1} \mathrm{H}$ NMR (400 MHz, $\left.\mathrm{CDCl}_{3}\right): \delta 7.28-7.21(2 \mathrm{H}$, m), 7.20-7.12 (3H, m), $5.23(1 \mathrm{H}, \mathrm{d}, \mathrm{J}=2.1 \mathrm{~Hz}), 5.04(1 \mathrm{H}, \mathrm{d}, \mathrm{J}=2.1 \mathrm{~Hz}), 4.14-4.07(1 \mathrm{H}, \mathrm{m})$, $3.77(4 \mathrm{H}, \mathrm{t}, \mathrm{J}=4.9 \mathrm{~Hz}), 3.45-3.39(1 \mathrm{H}, \mathrm{m}), 3.25-3.09(5 \mathrm{H}, \mathrm{m}), 2.92-2.81(2 \mathrm{H}, \mathrm{m}), 1.81-1.60$ $(6 \mathrm{H}, \mathrm{m})$. NH not observed. HRMS $\left(\mathrm{ESI}^{+}\right)(\mathrm{M}+\mathrm{H})^{+}$: calcd for $\mathrm{C}_{21} \mathrm{H}_{28} \mathrm{~N}_{3} \mathrm{O}_{2}, 354.2181$; found, 354.2216.

6-((R)-2-Benzylazepan-1-yl)-4-((R)-2-methylmorpholino)pyridin-2(1H)-one (56). LCMS $\left(\mathrm{ES}^{+}\right) 383(\mathrm{M}+\mathrm{H})^{+}$, RT $3.52 \mathrm{~min}$ (Analytical Method A); ${ }^{1} \mathrm{H}$ NMR (400 MHz, $\left.\mathrm{CDCl}_{3}\right): \delta 7.29$ - $7.12(5 \mathrm{H}, \mathrm{m}), 4.90(1 \mathrm{H}, \mathrm{s}), 4.70(1 \mathrm{H}, \mathrm{br} \mathrm{s}), 4.08(1 \mathrm{H}$, br s), $3.98-3.91(2 \mathrm{H}, \mathrm{m}), 3.67-3.54$ $(3 \mathrm{H}, \mathrm{m}), 3.00-2.86(3 \mathrm{H}, \mathrm{m}), 2.75-2.68(1 \mathrm{H}, \mathrm{m}), 2.61(1 \mathrm{H}, \mathrm{dd}, \mathrm{J}=10.5,13.0 \mathrm{~Hz}), 2.07-2.00$ $(1 \mathrm{H}, \mathrm{m}), 1.83-1.73(4 \mathrm{H}, \mathrm{m}), 1.48-1.41(2 \mathrm{H}, \mathrm{m}), 1.31-1.12(5 \mathrm{H}, \mathrm{m})$. HRMS $\left(\mathrm{ESI}^{+}\right)(\mathrm{M}+$ $\mathrm{H})^{+}$: calcd for $\mathrm{C}_{23} \mathrm{H}_{32} \mathrm{~N}_{3} \mathrm{O}_{2}$, 382.2494; found, 382.2516 .

(S)-6-(2-Benzyl-3,3-difluoropyrrolidin-1-yl)-4-morpholinopyridin-2(1H)-one (47). Step 1: Benzyl 2-benzyl-3-oxopyrrolidine-1-carboxylate. $\mathrm{KO} t \mathrm{Bu}(1.58 \mathrm{~g}, 14.04 \mathrm{mmol})$ was added to a solution of ((benzyloxy)carbonyl)-L-phenylalanine (4.0 g, $12.7 \mathrm{mmol})$ in anhydrous THF (30 $\mathrm{mL})$ at r.t. After $15 \mathrm{~min}$ a solution of methyl acrylate $(1.16 \mathrm{~mL}, 12.76 \mathrm{mmol})$ in anhydrous THF ( $5 \mathrm{~mL}$ ) was added dropwise and the resultant solution heated at reflux for $2.5 \mathrm{~h}$. The reaction mixture was cooled to r.t. and diluted with $1 \mathrm{M}$ aqueous $\mathrm{HCl}$. The solvent was removed under reduced pressure and the aqueous extracted with EtOAc. The organic extracts were 
washed with brine and dried $\left(\mathrm{MgSO}_{4}\right)$. The solvent was removed in vacuo to give an oil which was stirred vigorously with $0.3 \mathrm{M} \mathrm{HCl}$ at reflux for 4 days. The mixture was cooled to r.t. and the aqueous layer extracted with EtOAc. The organic layer was washed with $\mathrm{NaHCO}_{3}$ (saturated aqueous solution), brine and dried $\left(\mathrm{MgSO}_{4}\right)$ and concentrated under reduced pressure. The resultant residue was purified by silica gel column chromatography (gradient elution 0-50\% EtOAc/iso-hexane) to give the title compound. ${ }^{1} \mathrm{H} \mathrm{NMR}\left(400 \mathrm{MHz}, \mathrm{CDCl}_{3}\right): \delta$ 7.46-7.34 (8H, m), 7.03-6.91 (2H, m), 5.29-5.20 (2H, m), 4.36-4.18 (1H, m), 3.86-3.64 (1H, m), 3.51-3.01 (2H, m), 2.82-2.73 (1H, m), 2.45-2.34 (1H, m), 1.96-1.81 (1H, m).

Step 2: Benzyl 2-benzyl-3,3-difluoropyrrolidine-1-carboxylate. DAST (3.55 mmol) was added dropwise to a solution of benzyl 2-benzyl-3-oxopyrrolidine-1-carboxylate (500 mg, 1.62 mmol) in anhydrous DCM at $-78^{\circ} \mathrm{C}$ and the reaction allowed to warm to r.t. overnight. The reaction was cooled to $0{ }^{\circ} \mathrm{C}$ and quenched with $\mathrm{NaHCO}_{3}$ (saturated aqueous solution). The phases were separated, and the organic extracts dried (phase separator). The DCM layer was concentrated in vacuo. The crude was purified by silica gel column chromatography (gradient 0-50\% EtOAc/iso-hexane) to give the title compound. ${ }^{1} \mathrm{H} \mathrm{NMR}\left(400 \mathrm{MHz}, \mathrm{CDCl}_{3}\right): \delta 7.42-$ $7.36(7 \mathrm{H}, \mathrm{m}), 7.24-7.13(3 \mathrm{H}, \mathrm{m}), 5.35-5.07(3 \mathrm{H}, \mathrm{m}), 5.58-2.83(6 \mathrm{H}, \mathrm{m}), 2.21-2.07(1 \mathrm{H}, \mathrm{m})$, $1.82-1.63(1 \mathrm{H}, \mathrm{m})$.

Step 3: 2-Benzyl-3,3-difluoropyrrolidine. Following Method E starting from benzyl 2-benzyl3,3-difluoropyrrolidine-1-carboxylate $(515 \mathrm{mg}, 1.55 \mathrm{mmol})$. The reaction mixture was filtered through Celite and the solvent removed in vacuo to give the title compound, which was used without further purification. LCMS (ES $\left.{ }^{+}\right) 198(\mathrm{M}+\mathrm{H})^{+}$, RT 0.89 min (Analytical Method F); ${ }^{1} \mathrm{H}$ NMR $\left(400 \mathrm{MHz}, \mathrm{CDCl}_{3}\right): \delta$ 7.34-7.12 (5H, m), 3.42-3.13 (1H, m), 3.18-2.93 (3H, m), 2.68 (1H, dd, J=10.6, 14.0 Hz), 2.34-2.22 (2H, m). 
Step 4: 4-(2-(2-Benzyl-3,3-difluoropyrrolidin-1-yl)-6-((4-methoxybenzyl)oxy)pyridin-4yl)morpholine. Following Method D starting from 2-benzyl-3,3-difluoropyrrolidine (97 mg, $0.49 \mathrm{mmol})$ and 4-(2-chloro-6-((4-methoxybenzyl)oxy)pyridin-4-yl)morpholine (150 mg, 0.45 mmol). The residue was dissolved in DCM and washed with water. The organic extracts were dried (phase separator) and concentrated in vacuo. The residue was purified by silica gel column chromatography (gradient $0-50 \%$ EtOAc/iso-hexane) to give the title compound. LCMS (ES $\left.{ }^{+}\right) 496(\mathrm{M}+\mathrm{H})^{+}$, RT 1.88 min (Analytical Method F).

Step 5: (S)-6-(2-Benzyl-3,3-difluoropyrrolidin-1-yl)-4-morpholinopyridin-2(1H)-one (47). Following Method E starting from 4-(2-(2-benzyl-3,3-difluoropyrrolidin-1-yl)-6-((4methoxybenzyl)oxy)pyridin-4-yl)morpholine (208 $\mathrm{mg}, 0.42 \mathrm{mmol}$ ). The residue was purified by reverse phase preparative HPLC followed by separation of the enantiomers by SFC to afford the title compound (47). LCMS (ES $\left.{ }^{+}\right) 376(\mathrm{M}+\mathrm{H})^{+}$, RT 2.84 min (Analytical Method A); RT 2.09 min (Analytical Method SFC1,YMC AMYLOSE-C, 25/75 MeOH $\left.(0.1 \% \mathrm{DEA}) / \mathrm{CO}_{2}\right) ;{ }^{1} \mathrm{H}$ NMR (400 MHz, $\left.\mathrm{CDCl}_{3}\right): \delta$ 7.29-7.20 (3H, m), 7.17-7.15 (2H, m), $5.27(1 \mathrm{H}, \mathrm{d}, \mathrm{J}=2.2 \mathrm{~Hz})$, $4.80(1 \mathrm{H}, \mathrm{d}, \mathrm{J}=2.2 \mathrm{~Hz}), 4.35(1 \mathrm{H}, \mathrm{dt}, \mathrm{J}=4.6,18.2 \mathrm{~Hz}), 3.76(4 \mathrm{H}, \mathrm{t}, \mathrm{J}=4.6 \mathrm{~Hz}), 3.51(1 \mathrm{H}, \mathrm{dt}, \mathrm{J}=$ 2.2, $9.8 \mathrm{~Hz}), 3.42-3.35(1 \mathrm{H}, \mathrm{m}), 3.28-3.13(5 \mathrm{H}, \mathrm{m}), 2.88(1 \mathrm{H}, \mathrm{td}, \mathrm{J}=3,14.3 \mathrm{~Hz}), 2.32-2.22(1 \mathrm{H}$, m), 1.92-1.74 (2H, m). HRMS $\left(\mathrm{ESI}^{+}\right)(\mathrm{M}+\mathrm{H})^{+}$: calcd for $\mathrm{C}_{20} \mathrm{H}_{24} \mathrm{~F}_{2} \mathrm{~N}_{3} \mathrm{O}_{2}, 376.1836$; found, 376.1839 .

Data is given for the $(R)$-enantiomer of $\mathbf{4 7}$ for comparison:

(R)-6-(2-Benzyl-3,3-difluoropyrrolidin-1-yl)-4-morpholinopyridin-2(1H)-one; LCMS (ES $\left.{ }^{+}\right)$ $376(\mathrm{M}+\mathrm{H})^{+}$, RT 2.84 min (Analytical Method A); RT 2.99 min (Analytical Method SFC1,YMC AMYLOSE-C, 25/75 MeOH (0.1\%DEA)/CO 2$) ;{ }^{1} \mathrm{H}$ NMR (400 MHz, $\left.\mathrm{CDCl}_{3}\right): \delta$ 7.29-7.20 (3H, m), 7.17-7.15 (2H, m), $5.27(1 \mathrm{H}, \mathrm{d}, \mathrm{J}=2.2 \mathrm{~Hz}), 4.80(1 \mathrm{H}, \mathrm{d}, \mathrm{J}=2.2 \mathrm{~Hz}), 4.35$ $(1 \mathrm{H}, \mathrm{dt}, \mathrm{J}=4.6,18.2 \mathrm{~Hz}), 3.76(4 \mathrm{H}, \mathrm{t}, \mathrm{J}=5.1 \mathrm{~Hz}), 3.51(1 \mathrm{H}, \mathrm{dt}, \mathrm{J}=2.2,9.8 \mathrm{~Hz}), 3.42-3.35(1 \mathrm{H}$, m), 3.28-3.13 (5H, m), $2.88(1 \mathrm{H}, \mathrm{td}, \mathrm{J}=3,14.3 \mathrm{~Hz}), 2.32-2.22(1 \mathrm{H}, \mathrm{m}), 1.92-1.74(2 \mathrm{H}, \mathrm{m})$. 
6-((2S,3R)-2-Benzyl-3-fluoropyrrolidin-1-yl)-4-morpholinopyridin-2(1H)-one (48).

Step 1: Benzyl (2S*,3S*)-2-benzyl-3-hydroxypyrrolidine-1-carboxylate. Benzyl 2-benzyl-3oxopyrrolidine-1-carboxylate (from step 1 of compound 47, $613 \mathrm{mg}, 1.98 \mathrm{mmol}$ ) was dissolved in $\mathrm{MeOH}(0.2 \mathrm{M})$ and the resultant mixture cooled in an ice-bath. $\mathrm{NaBH}_{4}(1.1 \mathrm{eq})$ was added in one portion and the reaction allowed to warm to r.t. over $1 \mathrm{~h}$. The reaction was quenched by careful addition of water and the $\mathrm{MeOH}$ was removed under reduced pressure. The aqueous layer was extracted with DCM and the layers separated. The organic extracts were dried (phase separator) and concentrated under reduced pressure. The mixture was purified by silica gel column chromatography eluting with gradient $0-50 \%$ EtOAc/iso-hexane to afford the title compound. ${ }^{1} \mathrm{H}$ NMR $\left(400 \mathrm{MHz}, \mathrm{CDCl}_{3}\right): \delta 7.38-7.15(10 \mathrm{H}, \mathrm{m}), 5.20-4.96(2 \mathrm{H}, \mathrm{m}), 4.28(1 \mathrm{H}$, s), 4.14-4.08 (1H, m), 3.59-3.44 (2H, m), 3.01-2.87 (1H, m), 2.03-1.87 (2H, m), 1.81-1.73 (1H, $\mathrm{m}), 1.62-1.54(2 \mathrm{H}, \mathrm{m})$,

Step 2: Benzyl (2S*,3R*)-2-benzyl-3-fluoropyrrolidine-1-carboxylate. DAST (2.28 mmol) was added dropwise to a solution of benzyl (2S*,3 $\left.S^{*}\right)$-2-benzyl-3-hydroxypyrrolidine-1carboxylate (step 1 of compound $48,203 \mathrm{mg}, 0.65 \mathrm{mmol}$ ) in anhydrous $\mathrm{DCM}$ at $-78{ }^{\circ} \mathrm{C}$ and the reaction allowed to warm to r.t. overnight. The reaction was cooled in an ice bath and quenched by the addition of $\mathrm{NaHCO}_{3}$ (saturated aqueous solution). The phases were separated, and the organic extracts dried (phase separator). The DCM layer was concentrated in vacuo. The mixture was purified by silica gel column chromatography (gradient elution with gradient 050\% EtOAc/iso-hexane) to afford the title compound. ${ }^{1} \mathrm{H}$ NMR (400 $\left.\mathrm{MHz}, \mathrm{CDCl}_{3}\right): \delta 7.41$ $7.04(10 \mathrm{H}, \mathrm{m}), 5.23-5.14(2 \mathrm{H}, \mathrm{m}), 4.97-4.84(1 \mathrm{H}, \mathrm{m}), 4.38-4.19(1 \mathrm{H}, \mathrm{m}), 3.65-3.48(2 \mathrm{H}, \mathrm{m})$, 3.22-3.00 (1H, m), 2.64-2.48 (1H, m), 2.17-1.67 (2H, m). 
Step 3: (2S*,3R*)-2-Benzyl-3-fluoropyrrolidine. Following Method E starting from benzyl $\left(2 S^{*}, 3 R^{*}\right)$-2-benzyl-3-fluoropyrrolidine-1-carboxylate $(120 \mathrm{mg}, 0.38 \mathrm{mmol})$ to afford the title compound. ${ }^{1} \mathrm{H}$ NMR (400 MHz, $\left.\mathrm{CDCl}_{3}\right): \delta$ 7.39-7.20 (5H, m), $4.95(1 \mathrm{H}, \mathrm{dd}, \mathrm{J}=0.8,39.6 \mathrm{~Hz})$, 3.61-3.49 (1H, m), 3.05-3.18 (2H, m), 2.80-2.48 (3H, m), 2.08-1.97 (2H, m).

Step 4: 4-(2-((2S*,3R*)-2-Benzyl-3-fluoropyrrolidin-1-yl)-6-((4-methoxybenzyl)oxy)pyridin4-yl)morpholine. Following Method D starting from $\left(2 S^{*}, 3 R^{*}\right)$-2-benzyl-3-fluoropyrrolidine (43 mg, $0.24 \mathrm{mmol})$ and 4-(2-chloro-6-((4-methoxybenzyl)oxy)pyridin-4-yl)morpholine (73 $\mathrm{mg}, 0.22 \mathrm{mmol}$ ). The residue was purified by silica gel column chromatography (gradient 0 50\% EtOAc/iso-hexane) to give the title compound. LCMS $\left(\mathrm{ES}^{+}\right) 478(\mathrm{M}+\mathrm{H})^{+}, \mathrm{RT} 1.56 \mathrm{~min}$ (Analytical Method F).

Step 5: 6-((2S,3R)-2-Benzyl-3-fluoropyrrolidin-1-yl)-4-morpholinopyridin-2(1H)-one (48). Following Method E starting from 6-((2S*,3R*)-2-benzyl-3-fluoropyrrolidin-1-yl)-4morpholinopyridin-2(1H)-one $(100 \mathrm{mg}, 0.21 \mathrm{mmol})$. The residue was purified by reverse phase preparative HPLC followed by SFC to afford the title compound.

6-((2S,3R)-2-Benzyl-3-fluoropyrrolidin-1-yl)-4-morpholinopyridin-2(1H)-one (48); LCMS $\left(\mathrm{ES}^{+}\right) 358(\mathrm{M}+\mathrm{H})^{+}$, RT $2.77 \mathrm{~min}$ (Analytical Method A); RT $1.75 \mathrm{~min}$ (Analytical Method SFC4,YMC AMYLOSE-C, 30/70 MeOH (0.1\%DEAISO)/CO 2$) ;{ }^{1} \mathrm{H}$ NMR (400 MHz, $\left.\mathrm{CDCl}_{3}\right)$ : d 7.34-7.24 (3H, m), 7.18-7.16 (2H, m), $5.25(1 \mathrm{H}, \mathrm{d}, \mathrm{J}=2.2 \mathrm{~Hz}), 5.02(1 \mathrm{H}, \mathrm{dd}, \mathrm{J}=51.6,2.9 \mathrm{~Hz})$, $4.91(1 \mathrm{H}, \mathrm{d}, \mathrm{J}=2.2 \mathrm{~Hz}), 4.32(1 \mathrm{H}, \mathrm{dq}, \mathrm{J}=4.1,10.4 \mathrm{~Hz}), 3.79(4 \mathrm{H}, \mathrm{t}, \mathrm{J}=5.2 \mathrm{~Hz}), 3.63(1 \mathrm{H}, \mathrm{t}, \mathrm{J}=$ $9.2 \mathrm{~Hz}), 3.51(1 \mathrm{H}, \mathrm{dt}, \mathrm{J}=7.0,9.7 \mathrm{~Hz}), 3.24(4 \mathrm{H}, \mathrm{dd}, \mathrm{J}=4.1,5.9 \mathrm{~Hz}), 3.05-2.99(1 \mathrm{H}, \mathrm{m}), 2.70$ $(1 \mathrm{H}, \mathrm{dd}, \mathrm{J}=8.1,14.0 \mathrm{~Hz}), 2.26-2.16(1 \mathrm{H}, \mathrm{m}), 1.95-1.75(1 \mathrm{H}, \mathrm{m}), \mathrm{NH}$ not observed.

Data is shown for the $(2 R, 3 S)$-isomer for comparison: 
6-((2R,3S)-2-Benzyl-3-fluoropyrrolidin-1-yl)-4-morpholinopyridin-2(1H)-one; LCMS (ES $\left.{ }^{+}\right)$ $358(\mathrm{M}+\mathrm{H})^{+}$, RT 2.76 min (Analytical Method A); RT 2.23 min (Analytical Method SFC4,YMC AMYLOSE-C, 30/70 MeOH (0.1\%DEAISO)/CO 2$) ;{ }^{1} \mathrm{H}$ NMR (400 MHz, $\left.\mathrm{CDCl}_{3}\right)$ : 反 7.34-7.24 (3H, m), 7.18-7.16 (2H, m), $5.25(1 \mathrm{H}, \mathrm{d}, \mathrm{J}=2.2 \mathrm{~Hz}), 5.02(1 \mathrm{H}, \mathrm{dd}, \mathrm{J}=51.6,2.9 \mathrm{~Hz})$, $4.91(1 \mathrm{H}, \mathrm{d}, \mathrm{J}=2.2 \mathrm{~Hz}), 4.32(1 \mathrm{H}, \mathrm{dq}, \mathrm{J}=4.1,10.4 \mathrm{~Hz}), 3.79(4 \mathrm{H}, \mathrm{t}, \mathrm{J}=5.2 \mathrm{~Hz}), 3.63(1 \mathrm{H}, \mathrm{t}, \mathrm{J}=$ $9.2 \mathrm{~Hz}), 3.51(1 \mathrm{H}, \mathrm{dt}, \mathrm{J}=7.0,9.7 \mathrm{~Hz}), 3.24(4 \mathrm{H}, \mathrm{dd}, \mathrm{J}=4.1,5.9 \mathrm{~Hz}), 3.05-2.99(1 \mathrm{H}, \mathrm{m}), 2.70$ $(1 \mathrm{H}, \mathrm{dd}, \mathrm{J}=8.1,14.0 \mathrm{~Hz}), 2.26-2.16(1 \mathrm{H}, \mathrm{m}), 1.95-1.75(1 \mathrm{H}, \mathrm{m}), \mathrm{NH}$ not observed.

6-((2S,3R)-2-Benzyl-3-methoxypyrrolidin-1-yl)-4-morpholinopyridin-2(1H)-one (49).

Step 1: Benzyl (2S*,3R*)-3-(benzoyloxy)-2-benzylpyrrolidine-1-carboxylate. Benzoic acid (1.92 mmol) and triphenylphosphine $(1.92 \mathrm{mmol})$ were added to a solution of benzyl $\left(2 S^{*}, 3 S^{*}\right)$-2-benzyl-3-hydroxypyrrolidine-1-carboxylate (step 1 of compound $\mathbf{4 8}, 498 \mathrm{mg}, 1.60$ mmol $)$ in THF $(10 \mathrm{~mL})$ and cooled to $0{ }^{\circ} \mathrm{C}$. DIAD $(1.92 \mathrm{mmol})$ was added dropwise and the reaction allowed to warm to r.t. over $5 \mathrm{~h}$. The reaction mixture was concentrated in vacuo and purified by silica gel column chromatography (gradient elution, 0-50\% EtOAc/iso-hexane) to afford the title compound as a rotameric mixture: ${ }^{1} \mathrm{H}$ NMR $\left(400 \mathrm{MHz}, \mathrm{CDCl}_{3}\right): \delta 8.12-7.11$ (15H, m), 5.31-5.14 (3H, m), 4.35-4.23 (1H, m), 3.66-3.49 (2H, m), 3.21-3.03 (1H, m), 2.91$2.79(1 \mathrm{H}, \mathrm{m}), 2.04-1.98$ (1H. m), 1.89-1.83 (1H, m).

Step 2: Benzyl (2S*,3R*)-2-benzyl-3-hydroxypyrrolidine-1-carboxylate. Benzyl $\left(2 S^{*}, 3 R^{*}\right)-3$ (benzoyloxy)-2-benzylpyrrolidine-1-carboxylate (465 mg, $1.12 \mathrm{mmol}$ ) was dissolved in $\mathrm{MeOH}$ $(10 \mathrm{~mL})$ and water $(5 \mathrm{~mL})$. Lithium hydroxide monohydrate $(70.5 \mathrm{mg}, 1.68 \mathrm{mmol})$ was added in one portion and the reaction heated at $50{ }^{\circ} \mathrm{C}$ for $3 \mathrm{~h}$. The methanol was removed in vacuo and the aqueous layer was extracted with DCM. The layers were separated, dried (phase separator) and concentrated in vacuo. Purification by silica gel chromatography (gradient elution, 0-30\% EtOAc/iso-hexane) yielded the title compound. ${ }^{1} \mathrm{H} \mathrm{NMR}\left(400 \mathrm{MHz}, \mathrm{CDCl}_{3}\right)$ : $\delta$ 
7.48-7.05 (10H, m), 5.23-5.10 (2H, m), $4.18(1 \mathrm{H}, \mathrm{s}), 4.05-3.94(1 \mathrm{H}, \mathrm{m}), 3.70-3.45(2 \mathrm{H}, \mathrm{m})$, $3.15(1 \mathrm{H}, \mathrm{ddd}, \mathrm{J}=0.4,1.4,7.4 \mathrm{~Hz}), 2.53-2.47(1 \mathrm{H}, \mathrm{m}), 1.93-1.84(2 \mathrm{H}, \mathrm{m}), 1.51-1.47(1 \mathrm{H}, \mathrm{m})$.

Step 3: Benzyl (2S*,3R*)-2-benzyl-3-methoxypyrrolidine-1-carboxylate. NaH (77 mg, 1.93 mmol, $60 \%$ dispersion in mineral oil) was added in one portion to benzyl ( $\left.2 S^{*}, 3 R^{*}\right)-2$-benzyl3-hydroxypyrrolidine-1-carboxylate $(300 \mathrm{mg}, 0.96 \mathrm{mmol})$ in anhydrous DMF $(5 \mathrm{~mL})$ at $0{ }^{\circ} \mathrm{C}$. After $20 \mathrm{~min}$, methyl iodide $(0.09 \mathrm{~mL}, 1.45 \mathrm{mmol})$ was added and the reaction allowed to warm to r.t. over $2 \mathrm{~h}$. The reaction was cooled in an ice-bath and quenched by addition of water. The DMF was removed in vacuo and the residue dissolved in DCM. The DCM was washed with water and the layers separated. The organic extract was dried (phase separator) and concentrated in vacuo. The residue was purified by silica gel column chromatography (gradient 0-30\% EtOAc/iso-hexane) to give the title compound. LCMS $\left(\mathrm{ES}^{+}\right) 326(\mathrm{M}+\mathrm{H})^{+}, \mathrm{RT}$ 1.73 min (Analytical Method F); ${ }^{1} \mathrm{H}$ NMR (400 MHz, $\left.\mathrm{CDCl}_{3}\right): \delta 7.41-7.06(10 \mathrm{H}, \mathrm{m}), 5.23-5.12$ $(2 \mathrm{H}, \mathrm{m}), 4.15-4.02(2 \mathrm{H}, \mathrm{m}), 3.63-3.46(2 \mathrm{H}, \mathrm{m}), 3.23-3.01(4 \mathrm{H}, \mathrm{m}), 2.55-2.45(1 \mathrm{H}, \mathrm{m}), 1.98-$ $1.71(2 \mathrm{H}, \mathrm{m})$, rotamers observed.

Step 4: (2S*,3R*)-2-Benzyl-3-methoxypyrrolidine. Following Method E starting from racbenzyl $(2 R, 3 S)$-2-benzyl-3-methoxypyrrolidine-1-carboxylate $(250 \mathrm{mg}, 0.77 \mathrm{mmol})$. After filtering the reaction mixture through celite the solvent was removed in vacuo to give the title compound. Used without further purification.

Step $\quad 5: \quad 4-\left(2-\left(\left(2 S^{*}, 3 R^{*}\right)-2-B e n z y l-3-m e t h o x y p y r r o l i d i n-1-y l\right)-6-((4-\right.$ methoxybenzyl)oxy)pyridin-4-yl)morpholine. Following Method D starting from $\left(2 S^{*}, 3 R^{*}\right)$-2benzyl-3-methoxypyrrolidine $\quad(94 \quad \mathrm{mg}, \quad 0.49 \quad \mathrm{mmol}) \quad$ and $\quad 4-(2-c h l o r o-6-((4-$ methoxybenzyl)oxy)pyridin-4-yl)morpholine (150 mg, $0.45 \mathrm{mmol})$. The residue was purified by silica gel column chromatography (gradient $0-50 \%$ EtOAc/iso-hexane) to give the title compound. LCMS (ES $\left.{ }^{+}\right) 490(\mathrm{M}+\mathrm{H})^{+}$, RT $3.10 \min$ (Analytical Method F). 
Step 6: 6-((2S,3R)-2-Benzyl-3-methoxypyrrolidin-1-yl)-4-morpholinopyridin-2(1H)-one (49) and 6-((2R,3S)-2-benzyl-3-methoxypyrrolidin-1-yl)-4-morpholinopyridin-2(1H)-one. Following Method E starting from 4-(2-((2S*,3R*)-2-benzyl-3-methoxypyrrolidin-1-yl)-6((4-methoxybenzyl)oxy)pyridin-4-yl)morpholine $(190 \mathrm{mg}, 0.39 \mathrm{mmol})$. The residue was purified by reverse phase preparative HPLC. The sample was further purified by SFC chiral chromatography to give the title compound.

6-((2S,3R)-2-Benzyl-3-methoxypyrrolidin-1-yl)-4-morpholinopyridin-2(1H)-one (49); LCMS $\left(\mathrm{ES}^{+}\right) 370(\mathrm{M}+\mathrm{H})^{+}$, RT 2.85 min (Analytical Method A); RT 3.37 min (Analytical Method SFC4,YMC AMYLOSE-C, 30/70 IPA (0.1\%DEAISO)/CO 2$) ;{ }^{1} \mathrm{H}$ NMR (400 MHz, $\left.\mathrm{CDCl}_{3}\right): \delta$ 7.36-7.17 (5H, m), $5.21(1 \mathrm{H}, \mathrm{d}, \mathrm{J}=2.1 \mathrm{~Hz}), 4.88(1 \mathrm{H}, \mathrm{d}, \mathrm{J}=2.1 \mathrm{~Hz}), 4.07(1 \mathrm{H}, \mathrm{dd}, \mathrm{J}=4.1,8.5$ Hz), $3.78(4 \mathrm{H}, \mathrm{t}, \mathrm{J}=4.9 \mathrm{~Hz}), 3.74(1 \mathrm{H}, \mathrm{d}, \mathrm{J}=4.1 \mathrm{~Hz}), 3.51-3.39(2 \mathrm{H}, \mathrm{m}), 3.23(4 \mathrm{H}, \mathrm{dd}, \mathrm{J}=3.7$, $6.0 \mathrm{~Hz}), 3.16(3 \mathrm{H}, \mathrm{s}), 2.99(1 \mathrm{H}, \mathrm{dd}, \mathrm{J}=4.2,14.2 \mathrm{~Hz}), 2.66(1 \mathrm{H}, \mathrm{dd}, \mathrm{J}=8.5,14.2 \mathrm{~Hz}), 2.10-2.05$ $(1 \mathrm{H}, \mathrm{m}), 1.89-1.80(1 \mathrm{H}, \mathrm{m}), \mathrm{NH}$ not observed.

Data is given for the $(2 R, 3 S)$-isomer for comparison:

6-((2R,3S)-2-Benzyl-3-methoxypyrrolidin-1-yl)-4-morpholinopyridin-2(1H)-one; LCMS (ES $\left.{ }^{+}\right)$ $370(\mathrm{M}+\mathrm{H})^{+}$, RT 2.85 min (Analytical Method A); RT 2.52 min (Analytical Method SFC4,YMC AMYLOSE-C, 30/70 IPA (0.1\%DEAISO)/CO 2$) ;{ }^{1} \mathrm{H}$ NMR (400 MHz, $\left.\mathrm{CDCl}_{3}\right): \delta$ 7.36-7.17 $(5 \mathrm{H}, \mathrm{m}), 5.21(1 \mathrm{H}, \mathrm{d}, \mathrm{J}=2.1 \mathrm{~Hz}), 4.88(1 \mathrm{H}, \mathrm{d}, \mathrm{J}=2.1 \mathrm{~Hz}), 4.07(1 \mathrm{H}, \mathrm{dd}, \mathrm{J}=4.1,8.5$ Hz), $3.78(4 \mathrm{H}, \mathrm{t}, \mathrm{J}=4.9 \mathrm{~Hz}), 3.74(1 \mathrm{H}, \mathrm{d}, \mathrm{J}=4.0 \mathrm{~Hz}), 3.51-3.39(2 \mathrm{H}, \mathrm{m}), 3.23(4 \mathrm{H}, \mathrm{dd}, \mathrm{J}=3.7$, $6.0 \mathrm{~Hz}), 3.16(3 \mathrm{H}, \mathrm{s}), 2.99(1 \mathrm{H}, \mathrm{dd}, \mathrm{J}=4.2,14.2 \mathrm{~Hz}), 2.66(1 \mathrm{H}, \mathrm{dd}, \mathrm{J}=8.5,14.2 \mathrm{~Hz}), 2.10-2.05$ $(1 \mathrm{H}, \mathrm{m}), 1.89-1.80(1 \mathrm{H}, \mathrm{m}), \mathrm{NH}$ not observed.

6-((1S,2S,5R)-2-Benzyl-3-azabicyclo[3.1.0]hexan-3-yl)-4-((R)-2-methylmorpholino)pyridin$2(1 H)$-one (51). 
Step 1: Tert-butyl 3-azabicyclo[3.1.0]hexane-3-carboxylate. Boc anhydride (2.6 g, 11.9 mmol) and a solution of $\mathrm{NaHCO}_{3}(3.32 \mathrm{~g}, 39.5 \mathrm{mmol})$ in water $(78 \mathrm{~mL})$ was added to a suspension of 3-azabicyclo[3.1.0]hexane hydrochloride $(950 \mathrm{mg}, 7.9 \mathrm{mmol})$ in THF $(26 \mathrm{~mL})$. The resulting biphasic mixture was stirred vigorously overnight at r.t. The reaction mixture was diluted with water and DCM. The layers were separated and the organic phase dried (phase separator) and concentrated under reduced pressure. The residue was purified by silica gel column chromatography (gradient elution, $0-10 \%$ EtOAc/iso-hexane) to afford the title compound. ${ }^{1} \mathrm{H}$ NMR (400 MHz, $\left.\mathrm{CDCl}_{3}\right): \delta 3.55(1 \mathrm{H}, \mathrm{d}, \mathrm{J}=11.0 \mathrm{~Hz}), 3.47(1 \mathrm{H}, \mathrm{J}=11.0 \mathrm{~Hz}), 3.35-3.27(2 \mathrm{H}$, m), $1.42(9 \mathrm{H}, \mathrm{s}), 1.21-1.14(1 \mathrm{H}, \mathrm{m}), 0.93-0.84(1 \mathrm{H}, \mathrm{m}), 0.67-0.61(1 \mathrm{H}, \mathrm{m}), 0.16-0.13(1 \mathrm{H}, \mathrm{m})$. Step 2: Tert-butyl $\left(1 R^{*}, 2 R^{*}, 5 S^{*}\right)$-2-benzyl-3-azabicyclo[3.1.0]hexane-3-carboxylate and tertbutyl $\left(1 R^{*}, 2 S^{*}, 5 S^{*}\right)$-2-benzyl-3-azabicyclo[3.1.0]hexane-3-carboxylate. To a solution of tertbutyl 3-azabicyclo[3.1.0]hexane-3-carboxylate $(1.50 \mathrm{~g}, 7.9 \mathrm{mmol})$ in diethyl ether $(27 \mathrm{~mL})$, under $\mathrm{N}_{2}$ atmosphere, was added tetramethylethylenediamine $(1.23 \mathrm{~mL}, 8.2 \mathrm{mmol})$ and the reaction mixture cooled to $-78{ }^{\circ} \mathrm{C}$. sec-BuLi (11.8 $\mathrm{mL}, 10.6 \mathrm{mmol}, 0.9 \mathrm{M}$ in cyclohexane) was added and the mixture was stirred at $-78^{\circ} \mathrm{C}$ for $30 \mathrm{~min}$. Benzyl bromide (1.95 mL, $\left.16.4 \mathrm{mmol}\right)$ was added dropwise at $-78{ }^{\circ} \mathrm{C}$. After complete addition the reaction was allowed to slowly warm to r.t. overnight. The reaction was quenched with saturated aqueous $\mathrm{NH}_{4} \mathrm{Cl}$ solution, the layers separated and the aqueous further extracted with $\mathrm{Et}_{2} \mathrm{O}(\mathrm{x} 2)$. The combined organic extracts were dried $\left(\mathrm{MgSO}_{4}\right)$ and concentrated under reduced pressure. The crude material was purified by silica gel column chromatography (gradient elution, $0-10 \%$ EtOAc/iso-hexane) to afford the title compounds as separate diastereomers tert-butyl $\left(1 R^{*}, 2 S^{*}, 5 S^{*}\right)$-2-benzyl-3azabicyclo[3.1.0]hexane-3-carboxylate and tert-butyl $\left(1 R^{*}, 2 R^{*}, 5 S^{*}\right)-2$-benzyl-3azabicyclo[3.1.0]hexane-3-carboxylate. 
Isomer A $\left(592 \mathrm{mg}\right.$, later determined to be the $\left(1 R^{*}, 2 R^{*}, 5 S^{*}\right)$ isomer, see next step): LCMS $\left(\mathrm{ES}^{+}\right) 218\left(\mathrm{M}-{ }^{\mathrm{t}} \mathrm{Bu}\right)^{+}, \mathrm{RT} 1.77 \mathrm{~min}$ (Analytical Method F); ${ }^{1} \mathrm{H}$ NMR $\left(400 \mathrm{MHz}, \mathrm{CDCl}_{3}\right): \delta 7.31-$ $7.18(5 \mathrm{H}, \mathrm{m}), 4.12-3.96(1 \mathrm{H}, \mathrm{m}), 3.61-3.39(1 \mathrm{H}, \mathrm{m}), 3.22-2.91(2 \mathrm{H}, \mathrm{m}), 2.77-2.64(1 \mathrm{H}, \mathrm{m})$, 1.48-1.42 (9H, m), 1.38-1.28 (1H, m), 0.95-0.87 $(1 \mathrm{H}, \mathrm{m}), 0.59-0.53(1 \mathrm{H}, \mathrm{m}), 0.12-0.09(1 \mathrm{H}$, $\mathrm{m})$, rotamers observed.

Isomer B (420 mg, later determined to be the $\left(1 R^{*}, 2 S^{*}, 5 S^{*}\right)$ isomer, see next step): LCMS $\left(\mathrm{ES}^{+}\right) 218\left(\mathrm{M}-{ }^{\mathrm{t}} \mathrm{Bu}\right)^{+}, \mathrm{RT} 1.83 \min \left(\right.$ Analytical Method F); ${ }^{1} \mathrm{H} \mathrm{NMR}\left(400 \mathrm{MHz}, \mathrm{CDCl}_{3}\right): \delta 7.33-$ $7.27(4 \mathrm{H}, \mathrm{m}), 7.23-7.17(1 \mathrm{H}, \mathrm{m}), 4.04-3.95(1 \mathrm{H}, \mathrm{m}), 3.86-3.44(3 \mathrm{H}, \mathrm{m}), 2.40(1 \mathrm{H}, \mathrm{dd}, \mathrm{J}=10.8$, 12.4 Hz), 1.57-1.34 (11H, m), 0.69-0.63 (1H, m), 0.47-0.43 (1H, m), rotamers observed.

Step 3: $\left(1 R^{*}, 2 R^{*}, 5 S^{*}\right)-2-B e n z y l-3$-azabicyclo[3.1.0]hexane. To tert-butyl $\left(1 R^{*}, 2 R^{*}, 5 S^{*}\right)$-2benzyl-3-azabicyclo[3.1.0]hexane-3-carboxylate (Isomer A from previous step, $570 \mathrm{mg}, 2.09$ mmol) was added DCM ( $5 \mathrm{~mL})$ and TFA $(5 \mathrm{~mL})$ and the resulting mixture was stirred at r.t. for $1 \mathrm{~h}$. The solvent was evaporated and purified by SCX cartridge eluting sequentially with DCM:MeOH, 1:1 then 3:1 DCM:7N ammonia in methanol. The ammonia in methanol fractions were combined and concentrated under reduced pressure to afford the title compound. nOe experiments allowed determination of the stereochemistry of the benzyl group relative to the cyclopropyl group. ${ }^{1} \mathrm{H}$ NMR $\left(400 \mathrm{MHz}, \mathrm{CDCl}_{3}\right): \delta 7.32-7.17(5 \mathrm{H}, \mathrm{m}), 3.41(1 \mathrm{H}$, ddd, $\mathrm{J}=3.0,5.5,8.1 \mathrm{~Hz}), 2.94(2 \mathrm{H}, \mathrm{d}, \mathrm{J}=1.8 \mathrm{~Hz}), 2.82(1 \mathrm{H}, \mathrm{dd}, \mathrm{J}=5.9,13.0 \mathrm{~Hz}), 2.61(1 \mathrm{H}, \mathrm{dd}, \mathrm{J}=8.0$, $13.1 \mathrm{~Hz}), 1.38-1.32(1 \mathrm{H}, \mathrm{m}), 1.30-1.23(1 \mathrm{H}, \mathrm{m}), 0.40-0.34(1 \mathrm{H}, \mathrm{m}), 0.34-0.30(1 \mathrm{H}, \mathrm{m})$. NH not observed.

Step 4: (R)-4-(2,6-Dichloropyridin-4-yl)-2-methylmorpholine (Intermediate 38). Following Method B using (2R)-methyl morpholine $(0.5 \mathrm{~g}, 5 \mathrm{mmol})$. Purified by silica gel column chromatography (gradient elution, 10-50\% EtOAc/iso-hexane) to afford the title compound. ${ }^{1} \mathrm{H}$ NMR (400 MHz, $\left.\mathrm{CDCl}_{3}\right): \delta 6.57(2 \mathrm{H}, \mathrm{s}), 4.02(1 \mathrm{H}, \mathrm{ddt}, \mathrm{J}=1.3,5.1,5.8 \mathrm{~Hz}), 3.74-3.63(2 \mathrm{H}, \mathrm{m})$, 
3.58-3.49 (2H, m), $3.03(1 \mathrm{H}, \mathrm{dt}, \mathrm{J}=4.4,12.4 \mathrm{~Hz}), 2.67(1 \mathrm{H}, \mathrm{dd}, \mathrm{J}=10.4,12.4 \mathrm{~Hz}), 1.26(3 \mathrm{H}, \mathrm{d}$, $6.4 \mathrm{~Hz})$.

Step 5: (R)-4-(2-Chloro-6-((4-methoxybenzyl)oxy)pyridin-4-yl)-2-methylmorpholine (Intermediate 42). Following Method A using (R)-4-(2,6-dichloropyridin-4-yl)-2methylmorpholine (1.6 g, $6.4 \mathrm{mmol})$ and para-methoxybenzyl alcohol (0.87 mL, $7.0 \mathrm{mmol})$. Purified by silica gel column chromatography (gradient elution, 5-40\% EtOAc/iso-hexane) to afford the title compound. ${ }^{1} \mathrm{H}$ NMR $\left(400 \mathrm{MHz}, \mathrm{CDCl}_{3}\right)$ : $\delta 7.39-7.36(2 \mathrm{H}, \mathrm{m}), 6.91-6.88(2 \mathrm{H}$, m), $6.40(1 \mathrm{H}, \mathrm{d}, \mathrm{J}=2.0 \mathrm{~Hz}), 5.98(1 \mathrm{H}, \mathrm{d}, \mathrm{J}=2.0 \mathrm{~Hz}), 5.24(2 \mathrm{H}, \mathrm{s}), 4.00$ - $3.95(1 \mathrm{H}, \mathrm{m}), 3.81(3 \mathrm{H}$, s), $3.71-3.61(2 \mathrm{H}, \mathrm{m}), 3.52-3.42(2 \mathrm{H}, \mathrm{m}), 2.96-2.88(1 \mathrm{H}, \mathrm{m}), 2.58(1 \mathrm{H}, \mathrm{dd}, \mathrm{J}=10.4,12.4$ $\mathrm{Hz}), 1.24-1.21(3 \mathrm{H}, \mathrm{m})$.

Step $\quad 6: \quad(R)-4-\left(2-\left(\left(1 R^{*}, 2 R^{*}, 5 S^{*}\right)-2-b e n z y l-3-a z a b i c y c l o[3.1 .0] h e x a n-3-y l\right)-6-((4-\right.$ methoxybenzyl)oxy)pyridin-4-yl)-2-methylmorpholine. A mixture of (R)-6-chloro-4-(2methylmorpholino)pyridin-2(1H)-one (178 $\mathrm{mg}, 0.50 \mathrm{mmol}),\left(1 R^{*}, 2 R^{*}, 5 S^{*}\right)-2$-benzyl-3azabicyclo[3.1.0]hexane (88 mg, $0.50 \mathrm{mmol}), \mathrm{NaO} t \mathrm{Bu}(55 \mathrm{mg}, 0.55 \mathrm{mmol})$, RuPhos (12 mg, $0.025 \mathrm{mmol}$ ), and RuPhos palladacycle G1 methyl tert-butyl ether adduct ( $20 \mathrm{mg}, 0.025 \mathrm{mmol}$ ) were placed in a stem block tube and sealed. The tube was evacuated and refilled with nitrogen (x3). 1,4-Dioxane $(3.3 \mathrm{~mL})$ was added and the reaction heated at $85^{\circ} \mathrm{C}$ for $16 \mathrm{~h}$. The reaction mixture was cooled to r.t. and filtered through Celite. The mixture was concentrated under reduced pressure and purified by silica gel column chromatography (gradient elution, $5-40 \%$ EtOAc/iso-hexane) to afford the title compound as a yellow oil (230 mg, 95\%). LCMS (ES $)$ $486(\mathrm{M}+\mathrm{H})^{+}$, RT 1.77 min (Analytical Method F).

Step 7: 6-((1S,2S,5R)-2-Benzyl-3-azabicyclo[3.1.0]hexan-3-yl)-4-((R)-2methylmorpholino)pyridin-2(1H)-one (51). 
$(R)-4-\left(2-\left(\left(1 R^{*}, 2 R^{*}, 5 S^{*}\right)-2-\right.\right.$ benzyl-3-azabicyclo[3.1.0]hexan-3-yl)-6-((4-

methoxybenzyl)oxy)pyridin-4-yl)-2-methylmorpholine (220 mg, $0.45 \mathrm{mmol}$ ) was suspended in $\mathrm{EtOH}(4.5 \mathrm{~mL})$ and the reaction warmed to obtained a homogeneous solution. $5 \% \mathrm{Pd} / \mathrm{C}$ (220 mg, 100\% by wt.) was added followed by 1-methylcyclohexyl-1,4-diene (425 mg, $4.50 \mathrm{mmol}$ ). The reaction was heated at $75{ }^{\circ} \mathrm{C}$ for $3 \mathrm{~h}$. The reaction was cooled to r.t. and filtered through a plug of cotton to give a residue, which was purified by chiral SFC to yield $29 \mathrm{mg}(17 \%)$ of the title compound. Absolute configuration was assigned following determination of biological activity, the $(1 S)$ isomer being the more active. LCMS (ES+) 366 $(\mathrm{M}+\mathrm{H})+$, RT $3.05 \mathrm{~min}$ (Analytical Method A); RT $2.43 \mathrm{~min}$ (Analytical Method SFC4, YMC AMYLOSE-C, $20 / 80 \mathrm{MeOH}+0.1 \%$ DEAISO/CO2); ${ }^{1} \mathrm{H}$ NMR $\left(400 \mathrm{MHz}, \mathrm{CDCl}_{3}\right): \delta 7.35-$ $7.23(5 \mathrm{H}, \mathrm{m}), 5.28(1 \mathrm{H}, \mathrm{d}, \mathrm{J}=2.3 \mathrm{~Hz}), 5.02(1 \mathrm{H}, \mathrm{d}, \mathrm{J}=2.3 \mathrm{~Hz}), 4.00-3.92(2 \mathrm{H}, \mathrm{m}), 3.88(1 \mathrm{H}, \mathrm{d}$, $\mathrm{J}=9.1 \mathrm{~Hz}), 3.73-3.63(2 \mathrm{H}, \mathrm{m}), 3.54-3.42(3 \mathrm{H}, \mathrm{m}), 3.25(1 \mathrm{H}, \mathrm{dd}, \mathrm{J}=2.8,13.6 \mathrm{~Hz}), 2.97(1 \mathrm{H}$, dt, J=3.6, $12.3 \mathrm{~Hz}), 2.65-2.51(2 \mathrm{H}, \mathrm{m}), 1.73-1.67(2 \mathrm{H}, \mathrm{m}), 1.23(3 \mathrm{H}, \mathrm{d}, \mathrm{J}=6.1 \mathrm{~Hz}), 0.78(1 \mathrm{H}$, dt, J=5.1, 8.0 Hz), $0.62(1 \mathrm{H}, \mathrm{q}, \mathrm{J}=4.4 \mathrm{~Hz})$, NH not observed. HRMS $(\mathrm{ESI}+)(\mathrm{M}+\mathrm{H})+$ : calcd for $\mathrm{C} 22 \mathrm{H} 28 \mathrm{~N} 3 \mathrm{O} 2,366.2181$; found, 366.218 .

6-((1R,2S,5S)-2-Benzyl-3-azabicyclo[3.1.0]hexan-3-yl)-4-morpholinopyridin-2(1H)-one (52).

Step 1: $\left(1 R^{*}, 2 S^{*}, 5 S^{*}\right)-2-$ Benzyl-3-azabicyclo[3.1.0]hexane. To tert-butyl $\left(1 R^{*}, 2 S^{*}, 5 S^{*}\right)-2-$ benzyl-3-azabicyclo[3.1.0]hexane-3-carboxylate (Isomer B from step 2 of compound 51, 400 $\mathrm{mg}, 1.46 \mathrm{mmol})$ was added DCM $(3.5 \mathrm{~mL})$ and TFA $(3.5 \mathrm{~mL})$ and the resulting mixture was stirred at r.t. for $1 \mathrm{~h}$. The solvent was evaporated and purified by SCX cartridge eluting sequentially with DCM:MeOH, 1:1 then 3:1 DCM:7N ammonia in methanol. The ammonia in methanol fractions were combined and concentrated under reduced pressure to afford the title compound. nOe experiments allowed determination of the stereochemistry of the benzyl group relative to the cyclopropyl group. ${ }^{1} \mathrm{H} \mathrm{NMR}\left(400 \mathrm{MHz}, \mathrm{CDCl}_{3}\right): \delta 7.32-7.17(5 \mathrm{H}, \mathrm{m})$, 
$3.29(1 \mathrm{H}, \mathrm{t}, \mathrm{J}=7.2 \mathrm{~Hz}), 3.00(1 \mathrm{H}, \mathrm{dd}, \mathrm{J}=3.2,11.0 \mathrm{~Hz}), 2.87(1 \mathrm{H}, \mathrm{d}, \mathrm{J}=11.0 \mathrm{~Hz}), 2.69(1 \mathrm{H}, \mathrm{dd}$, $\mathrm{J}=7.2,13.5 \mathrm{~Hz}), 2.60(1 \mathrm{H}, \mathrm{dd}, \mathrm{J}=7.5,13.5 \mathrm{~Hz}), 1.43-1.36(1 \mathrm{H}, \mathrm{m}), 1.29(1 \mathrm{H}, \mathrm{ddd}, \mathrm{J}=4.0,6.2$, $7.9 \mathrm{~Hz}), 0.45(1 \mathrm{H}, \mathrm{dt}, \mathrm{J}=5.0,7.7 \mathrm{~Hz}), 0.15(1 \mathrm{H}, \mathrm{dd}, \mathrm{J}=4.0,8.7 \mathrm{~Hz})$. NH not observed.

Step 2: 4-(2,6-Dichloropyridin-4-yl)morpholine. 2,6-Dichloropyridin-4-amine (10 g, 61.3 mmol) was dissolved in DMF $(200 \mathrm{~mL})$ and cooled to $0{ }^{\circ} \mathrm{C}$. Sodium hydride $(6.13 \mathrm{~g}, 153$ mmol) was added portionwise over $20 \mathrm{~min}$. After $15 \mathrm{~min}$ 1-chloro-2-(2-chloroethoxy)ethane $(8.63 \mathrm{~mL}, 73.6 \mathrm{mmol})$ was added dropwise. The reaction mixture was warmed to r.t. and stirred for $21.5 \mathrm{~h}$. After this time the reaction was cooled to $0{ }^{\circ} \mathrm{C}$ and cautiously quenched with water. The DMF was removed under reduced pressure. The resultant residue was dissolved in DCM and washed with water. The organic layers were dried $\left(\mathrm{MgSO}_{4}\right)$, filtered and concentrated under reduced pressure. The crude product was triturated with EtOAc and iso-hexane to afford the title compound as a white solid $(9.27 \mathrm{~g}, 65 \%) .{ }^{1} \mathrm{H} \mathrm{NMR}\left(400 \mathrm{MHz}, \mathrm{CDCl}_{3}\right): \delta 6.59(2 \mathrm{H}, \mathrm{s})$, $3.84-3.80(4 \mathrm{H}, \mathrm{m}), 3.33-3.29(4 \mathrm{H}, \mathrm{m})$

Step 3: 4-(2-Chloro-6-((4-methoxybenzyl)oxy)pyridin-4-yl)morpholine. Following Method A using 4-(2,6-dichloropyridin-4-yl)morpholine (9.27 g, $39.8 \mathrm{mmol})$ and PMBOH (6.05 g, 43.8 mmol). The crude product was triturated with EtOAc and iso-hexane to afford the title compound as an off-white solid (11.4 g, 86\%). ${ }^{1} \mathrm{H}$ NMR (400 MHz, $\left.\mathrm{CDCl}_{3}\right): \delta 7.39-7.35(2 \mathrm{H}$, m), $6.91-6.88(2 \mathrm{H}, \mathrm{m}), 6.40(1 \mathrm{H}, \mathrm{d}, \mathrm{J}=2.0 \mathrm{~Hz}), 5.99(1 \mathrm{H}, \mathrm{d}, \mathrm{J}=2.0 \mathrm{~Hz}), 5.25(2 \mathrm{H}, \mathrm{s}), 3.81-$ $3.77(7 \mathrm{H}, \mathrm{m}), 3.25-3.21(4 \mathrm{H}, \mathrm{m})$.

Step 4: 4- $\quad$ 4- $\quad\left(\left(1 R^{*}, 2 S^{*}, 5 S^{*}\right)-2-b e n z y l-3-a z a b i c y c l o[3.1 .0]\right.$ hexan-3-yl)-6-((4methoxybenzyl)oxy)pyridin-4-yl)morpholine. A mixture of 4-(2-chloro-6-((4methoxybenzyl)oxy)pyridin-4-yl)morpholine (150 mg, $0.45 \mathrm{mmol}), \mathrm{NaOtBu}$ (48 mg, 0.50 mmol), RuPhos (11 mg, $0.023 \mathrm{mmol}$ ) and RuPhos Pd G1 catalyst (methyl t-butyl ether adduct, $18 \mathrm{mg}, 0.023 \mathrm{mmol})$ was placed in a tube and sparged with $\mathrm{N}_{2}$. A solution of $\left(1 R^{*}, 2 S^{*}, 5 S^{*}\right)-$ 
2-benzyl-3-azabicyclo[3.1.0]hexane $(78 \mathrm{mg}, 0.45 \mathrm{mmol})$ in anhydrous dioxane $(3 \mathrm{~mL})$ was added, the tube sealed, and stirring commenced at $85^{\circ} \mathrm{C}$ for $16 \mathrm{~h}$. The reaction was cooled to r.t. and filtered through Celite, washing with DCM, and the reaction concentrated under reduced pressure. The crude mixture was purified by silica gel column chromatography (gradient elution, 0-15\% EtOAc/iso-hexane) to afford the title compound as a green solid (197 mg, 93\%). LCMS (ES+) $472(\mathrm{M}+\mathrm{H})^{+}$, RT $1.48 \min$ (Analytical Method F).

Step 5: 6-((1R,2S,5S)-2-Benzyl-3-azabicyclo[3.1.0]hexan-3-yl)-4-morpholinopyridin-2(1H)one (52). Following Method E from 4-(2-((1R*,2S*,5 $\left.S^{*}\right)-2-$ benzyl-3-azabicyclo[3.1.0]hexan3-yl)-6-((4-methoxybenzyl)oxy)pyridin-4-yl)morpholine (190 mg, $0.40 \mathrm{mmol})$. Purification by silica gel column chromatography (gradient elution, 0-20\% MeOH/EtOAc) and chiral SFC yielded the title compound and its enantiomer. Absolute configuration was assigned following determination of biological activity, the $(1 R)$ isomer being the more active. LCMS (ES $\left.{ }^{+}\right) 352$ $(\mathrm{M}+\mathrm{H})^{+}$, RT 2.83 min (Analytical Method A); RT 1.65 min (Analytical Method SFC4,YMC AMYLOSE-C, 40/60 IPA + 0.1\% DEAISO/CO 2$) ;{ }^{1} \mathrm{H}$ NMR $\left(400 \mathrm{MHz}, \mathrm{CDCl}_{3}\right): \delta 7.27-7.26$ $(3 \mathrm{H}, \mathrm{m}), 7.19-7.15(2 \mathrm{H}, \mathrm{m}), 5.22(1 \mathrm{H}, \mathrm{d}, \mathrm{J}=2.0 \mathrm{~Hz}), 4.82(1 \mathrm{H}, \mathrm{d}, \mathrm{J}=2.0 \mathrm{~Hz}), 4.22(1 \mathrm{H}, \mathrm{dd}$, $\mathrm{J}=3.4,7.7 \mathrm{~Hz}), 3.81-3.77(4 \mathrm{H}, \mathrm{m}), 3.33(1 \mathrm{H}, \mathrm{d}, \mathrm{J}=9.3 \mathrm{~Hz}), 3.27-3.23(4 \mathrm{H}, \mathrm{m}), 3.12(1 \mathrm{H}, \mathrm{dd}$, $\mathrm{J}=4.0,9.3 \mathrm{~Hz}), 2.99(1 \mathrm{H}, \mathrm{dd}, \mathrm{J}=3.3,13.6 \mathrm{~Hz}), 2.81(1 \mathrm{H}, \mathrm{dd}, \mathrm{J}=8.0,13.6 \mathrm{~Hz}), 1.56-1.39(2 \mathrm{H}$, m), $0.66(1 \mathrm{H}, \mathrm{dt}, \mathrm{J}=5.2,7.8 \mathrm{~Hz}), 0.19(1 \mathrm{H}, \mathrm{q}, \mathrm{J}=4.3 \mathrm{~Hz})$, NH not observed. HRMS $\left(\mathrm{ESI}^{+}\right)(\mathrm{M}$ $+\mathrm{H})^{+}$: calcd for $\mathrm{C}_{21} \mathrm{H}_{26} \mathrm{~N}_{3} \mathrm{O}_{2}, 352.2025$; found, 352.203.

(R)-6-(2-Benzylazepan-1-yl)-4-morpholinopyridin-2(1H)-one (55).

Step 1: Tert-butyl (R)-(5-(2,2-dimethyl-4,6-dioxo-1,3-dioxan-5-yl)-5-oxo-1-phenylpentan-2yl)carbamate. (R)-4-((Tert-butoxycarbonyl)amino)-5-phenylpentanoic acid (3.0 g, $10.2 \mathrm{mmol})$ was dissolved in DCM (50 mL) and cooled to $0{ }^{\circ} \mathrm{C}$. Meldrum's acid (1.47 g, $\left.10.2 \mathrm{mmol}\right)$, EDC hydrochloride $(2.94 \mathrm{~g}, 15.3 \mathrm{mmol})$ and DMAP $(1.87 \mathrm{~g}, 15.3 \mathrm{mmol})$ were added sequentially to the reaction. The reaction mixture was stirred for $19 \mathrm{~h}$, removing the ice-bath after $1 \mathrm{~h}$. The 
reaction was transferred to a separating funnel and washed with $1 \mathrm{M}$ potassium bisulfate aqueous solution. The organic extracts were dried (phase separator) and concentrated under reduced pressure to afford the title compound. Used without further purification in the next step.

Step 2: Tert-butyl (R)-(5-(2,2-dimethyl-4,6-dioxo-1,3-dioxan-5-yl)-1-phenylpentan-2yl)carbamate. Tert-butyl $\quad(R)$-(5-(2,2-dimethyl-4,6-dioxo-1,3-dioxan-5-yl)-5-oxo-1phenylpentan-2-yl)carbamate $(2.1 \mathrm{~g}, 5.01 \mathrm{mmol})$ was dissolved in DCM (50 mL) and cooled to $0{ }^{\circ} \mathrm{C}$. Acetic acid $(3.16 \mathrm{~mL}, 55.1 \mathrm{mmol})$ was added followed by portionwise addition of $\mathrm{NaBH}_{4}(759 \mathrm{mg}, 20.05 \mathrm{mmol})$. The reaction was stirred at $0{ }^{\circ} \mathrm{C}$ for $3.5 \mathrm{~h}$. The reaction mixture was cautiously quenched by dropwise addition of water $(5 \mathrm{~mL})$ followed by brine $(\sim 100 \mathrm{~mL})$. The layers were separated, and the aqueous layer extracted with DCM. The combined layers were washed with brine, dried $\left(\mathrm{MgSO}_{4}\right)$, filtered and concentrated under reduced pressure to afford the title compound. Used without further purification in the next step.

Step 3: Tert-butyl (R)-6-((tert-butoxycarbonyl)amino)-7-phenylheptanoate. Tert-butyl (R)-(5(2,2-dimethyl-4,6-dioxo-1,3-dioxan-5-yl)-1-phenylpentan-2-yl)carbamate (1.86 g, 4.59 mmol) was dissolved in toluene $(5 \mathrm{~mL})$ and $t-\mathrm{BuOH}(5 \mathrm{~mL})$. The reaction mixture was refluxed for 6 h. After this time the solvents were removed under reduced pressure. The resultant oil was dissolved in toluene and heated at reflux for $25 \mathrm{~h}$. The reaction mixture was concentrated under reduced pressure. The oil was dissolved in $\mathrm{DCM}$ and $\mathrm{NaHCO}_{3}$ (saturated aqueous solution) added. The layers were inseparable. A small quantity of brine was added to separate the layers. The aqueous layer was extracted with EtOAc. The combined organic layers were dried (phase separator) and concentrated under reduced pressure. The crude oil was purified by silica gel column chromatography (gradient elution 0-30\% EtOAc/iso-hexane) to afford the title compound. ${ }^{1} \mathrm{H}$ NMR (400 MHz, $\left.\mathrm{CDCl}_{3}\right): \delta 7.32-7.14(5 \mathrm{H}, \mathrm{m}), 4.32(1 \mathrm{H}, \mathrm{d}, \mathrm{J}=0.8 \mathrm{~Hz}), 3.78$ 
(1H, br s), $3.32(2 \mathrm{H}, \mathrm{s}), 2.80-2.71(2 \mathrm{H}, \mathrm{m}), 2.60(2 \mathrm{H}, \mathrm{t}, \mathrm{J}=0.8 \mathrm{~Hz}), 1.83-1.81(1 \mathrm{H}, \mathrm{m}), 1.45$ $(9 \mathrm{H}, \mathrm{s}), 1.39(9 \mathrm{H}, \mathrm{s})$.

Step 4: (R)-6-Amino-7-phenylheptanoic acid. TFA $(2 \mathrm{~mL})$ was added to tert-butyl $(R)-6-(($ tertbutoxycarbonyl)amino)-7-phenylheptanoate (457 mg, $1.21 \mathrm{mmol})$ in DCM (2 mL) at r.t. with stirring. After $1.5 \mathrm{~h}$ the reaction mixture was concentrated under reduced pressure to afford a yellow oil. The oil was dissolved in $4 \mathrm{M} \mathrm{HCl}$ in dioxane $(10 \mathrm{~mL})$ and stirred at r.t. for $4 \mathrm{~h}$. The reaction mixture was concentrated under reduced pressure and dissolved in $4 \mathrm{M} \mathrm{HCl}$ in dioxane $(10 \mathrm{~mL})$. After $6 \mathrm{~h}$ the reaction mixture was concentrated under reduced pressure and used without further purification in the next step.

Step 5: (R)-7-Benzylazepan-2-one. EDC (348 mg, $1.82 \mathrm{mmol})$ and HOPO (201 mg, $1.82 \mathrm{mmol})$ were added to a solution of $(R)$-6-amino-7-phenylheptanoic acid $(1.21 \mathrm{mmol})$ and triethylamine $(1.01 \mathrm{~mL}, 7.26 \mathrm{mmol})$ in DMF $(13 \mathrm{~mL})$ at r.t. with stirring. After $54.5 \mathrm{~h}$ the reaction mixture was transferred to a separating funnel and diluted with DCM. The reaction mixture was washed sequentially with $1 \mathrm{M} \mathrm{HCl}(\mathrm{aq}), \mathrm{NaHCO}_{3}$ (saturated aqueous solution) and brine. The organics were dried $\left(\mathrm{MgSO}_{4}\right)$, filtered and concentrated under reduced pressure. The residue was purified by silica gel column chromatography (gradient elution 50-70\% EtOAc/iso-hexane) to afford the title compound. ${ }^{1} \mathrm{H} \mathrm{NMR}\left(400 \mathrm{MHz}, \mathrm{CDCl}_{3}\right): \delta 7.34-7.17(5 \mathrm{H}, \mathrm{m}), 5.54$ (1H, brs), 3.68-3.55 (1H, m), 2.87-2.72 (2H, m), 2.51-2.38 (2H, m), 1.98-1.81 (3H, m), 1.61-1.32 (3H, $\mathrm{m})$.

Step 6: (R)-2-Benzylazepane. Lithium aluminium hydride $(0.59 \mathrm{~mL}, 0.59 \mathrm{mmol}, 1 \mathrm{M}$ solution in THF) was added dropwise to an ice-cooled solution of $(R)$-7-benzylazepan-2-one (60 mg, $0.30 \mathrm{mmol})$ in THF $(2 \mathrm{~mL})$. The reaction mixture was warmed to r.t. and heated at reflux for $3 \mathrm{~h}$. The reaction mixture was cooled to r.t., followed by $0{ }^{\circ} \mathrm{C}$ and cautiously quenched with water. The THF was removed under reduced pressure. The mixture was diluted with water and EtOAc. The layers were separated and the organic extract dried $\left(\mathrm{MgSO}_{4}\right)$, filtered and 
concentrated under reduced pressure. The resultant oil was purified by SCX cartridge. The sample was loaded in DCM and eluted with 3 column volumes of DCM, 3 column volumes of methanol and 3 column volumes $7 \mathrm{~N} \mathrm{NH}_{3}$ in $\mathrm{MeOH} / \mathrm{DCM}(1: 9)$. The basic fractions were combined and concentrated under reduced pressure to afford the title compound. Used without further purification in the next step.

Step 7: (R)-4-(2-(2-benzylazepan-1-yl)-6-((4-methoxybenzyl)oxy)pyridin-4-yl)morpholine A mixture of 4-(2-chloro-6-((4-methoxybenzyl)oxy)pyridin-4-yl)morpholine (64 mg, 0.19 mmol), (R)-2-benzylazepane (40 mg, $0.21 \mathrm{mmol}$ ), NaOtBu (20 mg, 0.0.21 mmol), RuPhos (4 mg, $0.01 \mathrm{mmol}$ ) and RuPhos Pd G1 catalyst (methyl t-butyl ether adduct, $8 \mathrm{mg}, 0.01 \mathrm{mmol}$ ) was placed in a tube and sparged with $\mathrm{N}_{2}$. Anhydrous dioxane $(2.5 \mathrm{~mL})$ was added, the tube sealed, and stirring commenced at $85{ }^{\circ} \mathrm{C}$ for $16 \mathrm{~h}$. The reaction was cooled to r.t. and filtered through Celite, washing with DCM, and the reaction concentrated under reduced pressure. The crude mixture was purified by silica gel column chromatography (gradient elution, $10-100 \%$ $\mathrm{Et}_{2} \mathrm{O} /$ iso-hexane) to afford the title compound as a colourless oil (31 mg, 33\%).

${ }^{1} \mathrm{H}$ NMR (400 MHz, $\left.\mathrm{CDCl}_{3}\right): \delta 7.39$ (2H, m), 7.54-7.15 (5H, m), 6.91-6.87 (2H, m), $5.57(1 \mathrm{H}$, d, J=1.8Hz), $5.48(1 \mathrm{H}, \mathrm{d}, \mathrm{J}=1.8 \mathrm{~Hz}), 5.31(1 \mathrm{H}, \mathrm{dd}, \mathrm{J}=12.2,18.0 \mathrm{~Hz}), 3.82-3.76(7 \mathrm{H}, \mathrm{m}), 3.22-$ $3.17(4 \mathrm{H}, \mathrm{m}), 3.00-2.89$ (2H, m), 2.68 (1H, dd, J=8.6, $12.9 \mathrm{~Hz}), 1.99-1.90(1 \mathrm{H}, \mathrm{m}), 1.81-1.55$ $(5 \mathrm{H}, \mathrm{m}), 1.46-1.36(1 \mathrm{H}, \mathrm{m}), 1.30-1.09(3 \mathrm{H}, \mathrm{m})$.

Step 8: (R)-6-(2-Benzylazepan-1-yl)-4-morpholinopyridin-2(1H)-one (55). Following Method E from (R)-4-(2-(2-benzylazepan-1-yl)-6-((4-methoxybenzyl)oxy)pyridin-4-yl)morpholine (31 mg, $0.06 \mathrm{mmol}$ ). Purification by silica gel column chromatography (gradient elution, 0$20 \% \mathrm{MeOH} / \mathrm{EtOAc}$ ) yielded the title compound as an off-white solid (11 mg, $0.03 \mathrm{mmol}, 50 \%$ ).

LCMS (ES $) 368(\mathrm{M}+\mathrm{H})^{+}$, RT 2.91 min (Analytical Method A); RT 2.02 min (Analytical Method SFC1, YMC AMYLOSE-C, 35/65 IPA + 0.1\% DEAISO/CO $\left.{ }_{2}\right) ;{ }^{1} \mathrm{H}$ NMR (400 MHz, S56 
$\left.\mathrm{CDCl}_{3}\right): \delta 7.31-7.21(3 \mathrm{H}, \mathrm{m}), 7.16-7.12(2 \mathrm{H}, \mathrm{m}), 5.17(1 \mathrm{H}, \mathrm{d}, \mathrm{J}=2.0 \mathrm{~Hz}), 4.89(1 \mathrm{H}, \mathrm{d}, \mathrm{J}=2.0$ Hz), 3.80 - $3.75(6 \mathrm{H}, \mathrm{m}), 3.40$ - $3.35(1 \mathrm{H}, \mathrm{m}), 3.22$ - $3.18(4 \mathrm{H}, \mathrm{m}), 2.99(1 \mathrm{H}, \mathrm{dd}, \mathrm{J}=11.7,16.0$ Hz), $2.87-2.76(2 \mathrm{H}, \mathrm{m}), 2.15-2.05(1 \mathrm{H}, \mathrm{m}), 1.84-1.70(2 \mathrm{H}, \mathrm{m}), 1.57-1.45(2 \mathrm{H}, \mathrm{m}), 1.31$ $1.13(2 \mathrm{H}, \mathrm{m})$. HRMS $\left(\mathrm{ESI}^{+}\right)(\mathrm{M}+\mathrm{H})^{+}$: calcd for $\mathrm{C}_{22} \mathrm{H}_{30} \mathrm{~N}_{3} \mathrm{O}_{2}, 368.2338$; found, 368.2346. (R)-2-(2-Benzylazepan-1-yl)-6-morpholinopyrimidin-4(3H)-one (62). Step 1: (R)-4-(2-(2Benzylazepan-1-yl)-6-((4-methoxybenzyl)oxy)pyrimidin-4-yl)morpholine. 2-(R)Benzylazepane (169 mg, $0.89 \mathrm{mmol})$ was added to a solution of 4-(2-chloro-6-((4methoxybenzyl)oxy)pyrimidin-4-yl)morpholine (200 mg, $0.60 \mathrm{mmol})$ and DIPEA (156 $\mu \mathrm{L}$, $0.89 \mathrm{mmol})$ in DMF $(1.6 \mathrm{~mL})$. The reaction mixture was heated at $100{ }^{\circ} \mathrm{C}$. After $66.5 \mathrm{~h}$ the reaction mixture was cooled to r.t. and diluted with DCM and water. The layers were separated, and the organic extracts dried (phase separator). The crude mixture was purified by silica gel column chromatography (gradient elution, 10-80\% EtOAc/iso-hexane) to afford the title compound. ${ }^{1} \mathrm{H}$ NMR (400 MHz, $\left.\mathrm{CDCl}_{3}\right): \delta 7.35(2 \mathrm{H}, \mathrm{d}, \mathrm{J}=9.3 \mathrm{~Hz}), 7.28-7.35(5 \mathrm{H}, \mathrm{m}), 6.88$ $(2 \mathrm{H}, \mathrm{d}, \mathrm{J}=9.3 \mathrm{~Hz}), 5.37-5.23(3 \mathrm{H}, \mathrm{m}), 4.83-4.66(1 \mathrm{H}, \mathrm{m}), 4.29-4.18(1 \mathrm{H}, \mathrm{m}), 3.83-3.71(8 \mathrm{H}$, m), 3.55-3.44 (4H, m), 3.02-2.76 (2H, m), 2.69-2.57 (1H, m), 1.96-1.86 (1H, m), 1.78-1.11 $(6 \mathrm{H}, \mathrm{m})$.

Step 2: (R)-2-(2-Benzylazepan-1-yl)-6-morpholinopyrimidin-4(3H)-one (62). TFA (1 mL) was added to a solution of (R)-4-(2-(2-benzylazepan-1-yl)-6-((4-methoxybenzyl)oxy)pyrimidin-4yl)morpholine (144 mg, $0.295 \mathrm{mmol})$ in DCM (1 mL) at r.t. with stirring. After $5 \mathrm{~h}$ the reaction mixture was concentrated under reduced pressure. The crude residue was dissolved in DCM, washed with saturated $\mathrm{NaHCO}_{3}$ aqueous solution, dried (phase separator) and concentrated under reduced pressure. The resultant oil was dissolved in DMSO and purified by reverse phase preparative HPLC. The resultant solid was dissolved in DCM and washed with saturated aqueous solution of $\mathrm{NaHCO}_{3}$. The organic extracts were dried (phase separator) and concentrated under reduced pressure followed by freeze drying from $\mathrm{MeCN}$ :water to afford the 
title compound. LCMS (ES $) 369(\mathrm{M}+\mathrm{H})^{+}$, RT $3.26 \mathrm{~min}$ (Analytical Method A), ${ }^{1} \mathrm{H}$ NMR (400 $\left.\mathrm{MHz}, \mathrm{CDCl}_{3}\right): \delta 7.25-7.19(3 \mathrm{H}, \mathrm{m}), 7.16-7.11(2 \mathrm{H}, \mathrm{m}), 4.90(1 \mathrm{H}, \mathrm{s}), 4.71(1 \mathrm{H}, \mathrm{br} \mathrm{s}), 3.75-$ $3.60(5 \mathrm{H}, \mathrm{m}), 3.53-3.47(4 \mathrm{H}, \mathrm{m}), 2.92-2.82(2 \mathrm{H}, \mathrm{m}), 2.78-2.70(1 \mathrm{H}, \mathrm{m}), 2.08-1.98(1 \mathrm{H}$, m), 1.82 - $1.75(3 \mathrm{H}, \mathrm{m}), 1.49$ - $1.39(2 \mathrm{H}, \mathrm{m}), 1.28$ - $1.12(2 \mathrm{H}, \mathrm{m}), \mathrm{NH}$ not observed.

2-((R)-2-Benzylazepan-1-yl)-6-((R)-2-methylmorpholino)pyrimidin-4(3H)-one (63). Step 1: (R)-4-(2-((R)-2-Benzylazepan-1-yl)-6-((4-methoxybenzyl)oxy)pyrimidin-4-yl)-2-

methylmorpholine. (R)-4-(6-((4-Methoxybenzyl)oxy)-2-(methylsulfonyl)pyrimidin-4-yl)-2methylmorpholine (128 mg, $0.33 \mathrm{mmol}),(R)$-2-benzylazepane (40 mg, $0.21 \mathrm{mmol})$ and TBAF hydrate $(131 \mathrm{mg}, 0.50 \mathrm{mmol})$ were suspended in DMSO $(0.7 \mathrm{~mL})$. The reaction was heated at $80{ }^{\circ} \mathrm{C}$ for 6 days. After $42 \mathrm{~h}$ at $80{ }^{\circ} \mathrm{C}$, further TBAF hydrate $(50 \mathrm{mg}, 0.19 \mathrm{mmol})$ was added, and after $68 \mathrm{~h}$ at $80{ }^{\circ} \mathrm{C}$ DIPEA $(120 \mu \mathrm{L}, 0.69 \mathrm{mmol})$ was added to the reaction. On completion of the reaction, and after cooling to r.t., water $(2 \mathrm{~mL})$ was added, whereupon a gum formed. The liquid supernatant was decanted off and the gum dissolved in a 1:1 mixture of DCM and water. The biphasic mixture was separated by passage through a hydrophobic frit; the DCM layer was concentrated to give the title compound, which was used without further purification.

Step 2: 2-((R)-2-Benzylazepan-1-yl)-6-((R)-2-methylmorpholino)pyrimidin-4(3H)-one (63). Following Method $\quad$ H $\quad$ from $\quad(R)-4-(2-((R)-2-b e n z y l a z e p a n-1-y)-6-((4-$ methoxybenzyl)oxy)pyrimidin-4-yl)-2-methylmorpholine. The reaction was stirred at r.t. for 4.5 h. The reaction was quenched with $\mathrm{MeOH}$ and concentrated. Purification by reverse phase preparative HPLC was followed by freeze-drying gave the title compound. LCMS (ES $) 383$ $(\mathrm{M}+\mathrm{H})^{+}$, RT $3.52 \min \left(\right.$ Analytical Method A); ${ }^{1} \mathrm{H}$ NMR (400 MHz, $\left.\mathrm{CDCl}_{3}\right): \delta 7.29-7.12(5 \mathrm{H}$, m), $4.90(1 \mathrm{H}, \mathrm{s}), 4.70(1 \mathrm{H}$, br s), $4.08(1 \mathrm{H}, \mathrm{br} \mathrm{s}), 3.98$ - $3.91(2 \mathrm{H}, \mathrm{m}), 3.67$ - $3.54(3 \mathrm{H}, \mathrm{m}), 3.00$ - $2.86(3 \mathrm{H}, \mathrm{m}), 2.75-2.68(1 \mathrm{H}, \mathrm{m}), 2.61(1 \mathrm{H}, \mathrm{dd}, \mathrm{J}=10.5,13.0 \mathrm{~Hz}), 2.07-2.00(1 \mathrm{H}, \mathrm{m}), 1.83$ 
- $1.73(4 \mathrm{H}, \mathrm{m}), 1.48-1.41(2 \mathrm{H}, \mathrm{m}), 1.31-1.12(5 \mathrm{H}, \mathrm{m})$. HRMS $\left(\mathrm{ESI}^{+}\right)(\mathrm{M}+\mathrm{H})^{+}$: calcd for $\mathrm{C}_{22} \mathrm{H}_{31} \mathrm{~N}_{4} \mathrm{O}_{2}$, 383.2447; found, 383.2447.

The compounds listed below were synthesised using the same synthetic route as described for compound 63, starting from commercially available amines and either 4-(6-((4methoxybenzyl)oxy)-2-(methylthio)pyrimidin-4-yl)morpholine $\quad$ or $\quad(R)-4-(6-((4-$ methoxybenzyl)oxy)-2-(methylsulfonyl)pyrimidin-4-yl)-2-methylmorpholine. Methyl sulfonyl displacement conditions Method F or Method G were used, followed by Method H to remove the PMB protecting group. The isomers were isolated after purification by Chiral SFC unless indicated (*), in which cases the amine was used chirally pure in the synthesis.

2-((1R,2S, 5S)-2-Benzyl-3-azabicyclo[3.1.0]hexan-3-yl)-6-morpholinopyrimidin-4(3H)-one

(64). Method G. LCMS (ES $) 353(\mathrm{M}+\mathrm{H})^{+}$, RT $3.31 \mathrm{~min}$ (Analytical Method A), RT $2.71 \mathrm{~min}$ (Analytical Method SFC1, YMC AMYLOSE-C 40/60 IPA [0.1\% DEAISO]/CO ${ }_{2}$ ); ${ }^{1} \mathrm{H}$ NMR $\left(400 \mathrm{MHz}, \mathrm{CDCl}_{3}\right): \delta 7.32-7.26(2 \mathrm{H}, \mathrm{m}), 7.25-7.16(3 \mathrm{H}, \mathrm{m}), 4.94(1 \mathrm{H}, \mathrm{s}), 4.49(1 \mathrm{H}, \mathrm{dd}$, $\mathrm{J}=3.0,7.8 \mathrm{~Hz}), 3.75(4 \mathrm{H}, \mathrm{dd}, \mathrm{J}=4.9,4.9 \mathrm{~Hz}), 3.59-3.48(5 \mathrm{H}, \mathrm{m}), 3.26(1 \mathrm{H}, \mathrm{dd}, \mathrm{J}=3.0,10.1 \mathrm{~Hz})$ 3.12 - $3.06(1 \mathrm{H}, \mathrm{m}), 2.80(1 \mathrm{H}, \mathrm{dd}, \mathrm{J}=8.5,13.0 \mathrm{~Hz}), 1.50-1.43(2 \mathrm{H}, \mathrm{m}), 0.68-0.61(1 \mathrm{H}, \mathrm{m})$, $0.18-0.13(1 \mathrm{H}, \mathrm{m}), \mathrm{NH}$ not observed. HRMS $\left(\mathrm{ESI}^{+}\right)(\mathrm{M}+\mathrm{H})^{+}$: calcd for $\mathrm{C}_{20} \mathrm{H}_{25} \mathrm{~N}_{4} \mathrm{O}_{2}$, 353.1977; found, 353.1976.

2-((1R,2S, 5S)-2-Benzyl-3-azabicyclo[3.1.0]hexan-3-yl)-6-((R)-2-

methylmorpholino)pyrimidin-4(3H)-one (65). Method G. LCMS (ES $\left.{ }^{+}\right) 367(\mathrm{M}+\mathrm{H})^{+}, \mathrm{RT} 3.45$ min (Analytical Method A), RT 2.48 min (Analytical Method SFC1, YMC AMYLOSE-C 35/65 IPA [0.1\% DEAISO $\left./ \mathrm{CO}_{2}\right) ;{ }^{1} \mathrm{H}$ NMR (400 MHz, $\left.\mathrm{CDCl}_{3}\right): \delta 10.42$ - $10.42(1 \mathrm{H}$, br s), $7.31-7.26(2 \mathrm{H}, \mathrm{m}), 7.25-7.18(3 \mathrm{H}, \mathrm{m}), 4.94(1 \mathrm{H}, \mathrm{s}), 4.52(1 \mathrm{H}, \mathrm{dd}, \mathrm{J}=3.6,8.7 \mathrm{~Hz}), 4.08$ - 3.94 $(3 \mathrm{H}, \mathrm{m}), 3.68$ - $3.56(3 \mathrm{H}, \mathrm{m}), 3.35-3.34(1 \mathrm{H}, \mathrm{m}), 3.14(1 \mathrm{H}, \mathrm{d}, \mathrm{J}=12.4 \mathrm{~Hz}), 2.97(1 \mathrm{H}, \mathrm{dt}, \mathrm{J}=3.1$, $12.4 \mathrm{~Hz}), 2.74(1 \mathrm{H}, \mathrm{dd}, \mathrm{J}=8.8,13.2 \mathrm{~Hz}), 2.63(1 \mathrm{H}, \mathrm{dd}, \mathrm{J}=10.5,12.4 \mathrm{~Hz}), 1.61-1.44(2 \mathrm{H}, \mathrm{m})$, 
$\left.1.23(3 \mathrm{H}, \mathrm{d}, \mathrm{J}=6.1 \mathrm{~Hz}), 0.67-0.60(1 \mathrm{H}, \mathrm{m}), 0.19-0.14(1 \mathrm{H}, \mathrm{m}) . \mathrm{HRMS}_{(\mathrm{ESI}}^{+}\right)(\mathrm{M}+\mathrm{H})^{+}$: calcd for $\mathrm{C}_{21} \mathrm{H}_{27} \mathrm{~N}_{4} \mathrm{O}_{2}, 367.2134$; found, 367.2141.

2-((1S,2S,5R)-2-Benzyl-3-azabicyclo[3.1.0]hexan-3-yl)-6-morpholinopyrimidin-4(3H)-one

(66). Method G. LCMS (ES $\left.{ }^{+}\right) 353(\mathrm{M}+\mathrm{H})^{+}$, RT 3.16 min (Analytical Method B), RT 8.50 min (Analytical Method SFC1, LUX CELLULOSE-4 30/70 MeOH [0.1\% DEAISO ]/CO ${ }_{2}$ ); ${ }^{1} \mathrm{H}$ $\operatorname{NMR}\left(400 \mathrm{MHz}, \mathrm{CDCl}_{3}\right): \delta 7.34-7.21(5 \mathrm{H}, \mathrm{m}), 5.00(1 \mathrm{H}, \mathrm{s}), 4.42$ - $4.36(1 \mathrm{H}, \mathrm{m}), 3.85$ - 3.74 $(6 \mathrm{H}, \mathrm{m}), 3.64-3.52(5 \mathrm{H}, \mathrm{m}), 2.40(1 \mathrm{H}, \mathrm{dd}, \mathrm{J}=10.4,12.6 \mathrm{~Hz}), 1.74-1.66(1 \mathrm{H}, \mathrm{m}), 1.58-1.50$ $(1 \mathrm{H}, \mathrm{m}), 0.82-0.75(1 \mathrm{H}, \mathrm{m}), 0.58(1 \mathrm{H}, \mathrm{q}, \mathrm{J}=4.5 \mathrm{~Hz}), \mathrm{NH}$ not observed. HRMS $\left(\mathrm{ESI}^{+}\right)(\mathrm{M}+$ $\mathrm{H})^{+}$: calcd for $\mathrm{C}_{20} \mathrm{H}_{25} \mathrm{~N}_{4} \mathrm{O}_{2}$, 353.1977; found, 353.1986 .

2-((1S,2S,5R)-2-Benzyl-3-azabicyclo[3.1.0]hexan-3-yl)-6-((R)-2-

methylmorpholino)pyrimidin-4(3H)-one (67). Method G. LCMS (ES $\left.{ }^{+}\right) 367(\mathrm{M}+\mathrm{H})^{+}, \mathrm{RT} 3.32$ min (Analytical Method A), RT 7.68 min (Analytical Method SFC1, LUX CELLULOSE-4 30/70 MeOH [0.1\% DEAISO ]/CO 2 ); ${ }^{1} \mathrm{H}$ NMR (400 MHz, $\left.\mathrm{CDCl}_{3}\right): \delta 10.32$ - $10.32(1 \mathrm{H}$, br s), $7.32-7.29(4 \mathrm{H}, \mathrm{m}), 7.25-7.21(1 \mathrm{H}, \mathrm{m}), 5.00(1 \mathrm{H}, \mathrm{s}), 4.43-4.36(1 \mathrm{H}, \mathrm{m}), 4.21(1 \mathrm{H}, \mathrm{d}, \mathrm{J}=11.9$ Hz), 4.04 - $3.94(2 \mathrm{H}, \mathrm{m}), 3.89(2 \mathrm{H}, \mathrm{d}, \mathrm{J}=9.5 \mathrm{~Hz}), 3.83(1 \mathrm{H}, \mathrm{dd}, \mathrm{J}=3.4,12.9 \mathrm{~Hz}), 3.70$ - 3.56 $(2 \mathrm{H}, \mathrm{m}), 3.01(1 \mathrm{H}, \mathrm{dt}, \mathrm{J}=3.5,12.4 \mathrm{~Hz}), 2.67(1 \mathrm{H}, \mathrm{dd}, \mathrm{J}=10.5,13.0 \mathrm{~Hz}), 2.38(1 \mathrm{H}, \mathrm{dd}, \mathrm{J}=10.6$, $12.6 \mathrm{~Hz}), 1.74-1.66(1 \mathrm{H}, \mathrm{m}), 1.57-1.50(1 \mathrm{H}, \mathrm{m}), 1.22(3 \mathrm{H}, \mathrm{d}, \mathrm{J}=6.3 \mathrm{~Hz}), 0.82-0.75(1 \mathrm{H}, \mathrm{m})$, $0.59(1 \mathrm{H}, \mathrm{q}, \mathrm{J}=4.4 \mathrm{~Hz}) . \quad \mathrm{HRMS}\left(\mathrm{ESI}^{+}\right)(\mathrm{M}+\mathrm{H})^{+}$: calcd for $\mathrm{C}_{21} \mathrm{H}_{27} \mathrm{~N}_{4} \mathrm{O}_{2}, 367.2134$; found, 367.2139 .

2-((2S,3R)-2-Benzyl-3-methylpyrrolidin-1-yl)-6-morpholinopyrimidin-4(3H)-one (68)

Step 1: Tert-butyl (2R*,3S*)-2-benzyl-3-methylpyrrolidine-1-carboxylate. Palladium (II) acetate (18 mg, $0.08 \mathrm{mmol}$, Dpe-phos $(86 \mathrm{mg}, 0.16 \mathrm{mmol})$ and sodium tert-butoxide $(884 \mathrm{mg}$, $9.2 \mathrm{mmol})$ were placed in a reaction tube sealed, evacuated and re-filled with nitrogen $(\mathrm{x} 3) .3-$ Methylpent-4-en-1-yl)carbamate (798 mg, $4.0 \mathrm{mmol})$ was dissolved in dioxane $(16 \mathrm{~mL})$ and degassed. The solution was added to the reaction tube followed by bromobenzene $(0.25 \mathrm{~mL}$, 
$4.8 \mathrm{mmol}$ ) and the reaction heated at $100{ }^{\circ} \mathrm{C}$ for $17.5 \mathrm{~h}$. The reaction mixture was cooled to r.t., diluted with $\mathrm{NH}_{4} \mathrm{Cl}$. The reaction mixture was extracted with EtOAc (x3). The organic extracts dried (phase separator) and concentrated under reduced pressure. The crude product was purified by silica gel column chromatography (gradient elution, 2-20\% EtOAc/iso-hexane) to afford the title compound. ${ }^{1} \mathrm{H}$ NMR $\left(400 \mathrm{MHz}, \mathrm{CDCl}_{3}\right): \delta 7.31-7.13(5 \mathrm{H}, \mathrm{m}), 3.68-3.36(2 \mathrm{H}$, m), 3.27-2.98 (2H, m), 2.79-2.57 (1H, m), 2.11-1.76 (2H, m), 1.61-1.46 (9H, m), 0.95-0.81 $(4 \mathrm{H}, \mathrm{m})$, rotamers observed.

Step 2: $\quad\left(2 R^{*}, 3 S^{*}\right)$-2-Benzyl-3-methylpyrrolidine. $\quad$ Tert-butyl $\quad\left(2 R^{*}, 3 S^{*}\right)-2$-benzyl-3methylpyrrolidine-1-carboxylate $(785 \mathrm{mg}, 2.85 \mathrm{mmol}$ ) was dissolved in $4 \mathrm{M} \mathrm{HCl}$ in dioxane $(7.1 \mathrm{~mL})$ at r.t. with stirring. After $2 \mathrm{~h}$ the reaction mixture was concentrated under reduced pressure. The residue was dissolved in DCM and washed with saturated $\mathrm{NaHCO}_{3}$ solution. The organic extract was dried (phase separator) and concentrated under reduced pressure to afford the title compound. ${ }^{1} \mathrm{H}$ NMR $\left(400 \mathrm{MHz}, \mathrm{CDCl}_{3}\right): \delta 7.32-7.16(5 \mathrm{H}, \mathrm{m}), 3.00-2.79(3 \mathrm{H}$, m), 2.77 - $2.68(1 \mathrm{H}, \mathrm{m}), 2.59(1 \mathrm{H}, \mathrm{dd}, \mathrm{J}=8.5,13.3 \mathrm{~Hz}), 2.08-1.96(1 \mathrm{H}, \mathrm{m}), 1.76-1.66(1 \mathrm{H}$, m), $1.42-1.31(1 \mathrm{H}, \mathrm{m}), 1.00(3 \mathrm{H}, \mathrm{d}, \mathrm{J}=6.6 \mathrm{~Hz}), \mathrm{NH}$ not observed.

Step 3: 4-(6-((4-Methoxybenzyl)oxy)-2-(methylthio)pyrimidin-4-yl)morpholine. NaH (3.43 g, $85.8 \mathrm{mmol}, 60 \%$ dispersion in mineral oil) was suspended in DMF $(70 \mathrm{~mL})$ and cooled to $0{ }^{\circ} \mathrm{C}$. 4-methoxy benzyl alcohol (6.52 g, $47.2 \mathrm{mmol})$ in DMF (30 $\mathrm{mL})$ was added dropwise to the suspension over 15 minutes. After stirring at $0{ }^{\circ} \mathrm{C}$ for $15 \mathrm{~min}$ a solution of 4 -(6-chloro-2(methylthio)pyrimidin-4-yl)morpholine (10.54 g, $42.9 \mathrm{mmol}$, prepared according to WO 2008/125839) in DMF (90 mL) was added. The reaction was allowed to warm to r.t. and stirred for $4.5 \mathrm{~h}$. The reaction was cooled to $0{ }^{\circ} \mathrm{C}$ and cautiously quenched with water. The reaction mixture was concentrated under reduced pressure. The residue was dissolved in EtOAc and washed with brine and water. The organic extracts were dried $\left(\mathrm{MgSO}_{4}\right)$, filtered and 
concentrated under reduced pressure. Purified by silica gel column chromatography (gradient elution, 10-50\% EtOAc/iso-hexane) to afford the title compound. LCMS (ES $\left.{ }^{+}\right) 348(\mathrm{M}+\mathrm{H})^{+}$, RT 1.69 min (Analytical Method F); ${ }^{1} \mathrm{H}$ NMR (400 MHz, $\left.\mathrm{CDCl}_{3}\right): \delta$ 7.37-7.33 (2H, m), 6.91$6.87(2 \mathrm{H}, \mathrm{m}), 5.53(1 \mathrm{H}, \mathrm{s}), 5.30(2 \mathrm{H}, \mathrm{s}), 3.80(3 \mathrm{H}, \mathrm{s}), 3.76-3.72(4 \mathrm{H}, \mathrm{m}), 3.55-3.51(4 \mathrm{H}, \mathrm{m})$, $2.51(3 \mathrm{H}, \mathrm{s})$.

Step 4: 4-(6-((4-Methoxybenzyl)oxy)-2-(methylsulfonyl)pyrimidin-4-yl)morpholine. m-CPBA (759 mg, $4.4 \mathrm{mmol}, 50-55$ wt.\% in $\left.\mathrm{H}_{2} \mathrm{O}\right)$ was added portionwise to a solution of 4-(6-((4methoxybenzyl)oxy)-2-(methylthio)pyrimidin-4-yl)morpholine (695 mg, $2.0 \mathrm{mmol})$ in DCM $(20 \mathrm{~mL})$ at r.t. After $2 \mathrm{~h}$ the reaction was filtered, washing the precipitate with DCM. The filtrate was washed successively with saturated $\mathrm{NaHCO}_{3}$ (aqueous solution), brine, dried (phase separator) and concentrated under reduced pressure to afford the product, which was used without further purification in the next step. ${ }^{1} \mathrm{H}$ NMR $\left(400 \mathrm{MHz}, \mathrm{CDCl}_{3}\right): \delta 7.38-7.35$ $(2 \mathrm{H}, \mathrm{m}), 6.91-6.88(2 \mathrm{H}, \mathrm{m}), 5.88(1 \mathrm{H}, \mathrm{s}), 5.36(2 \mathrm{H}, \mathrm{s}), 3.81(3 \mathrm{H}, \mathrm{s}), 3.78-3.74(4 \mathrm{H}, \mathrm{m}), 3.63$ - $3.57(4 \mathrm{H}, \mathrm{m}), 3.26(3 \mathrm{H}, \mathrm{s})$.

Step $\quad 5: \quad 4-\left(2-\left(\left(2 R^{*}, 3 S^{*}\right)-2-b e n z y l-3-m e t h y l p y r r o l i d i n-1-y l\right)-6-((4-\right.$ methoxybenzyl)oxy)pyrimidin-4-yl)morpholine. $\quad$ A suspension of 4-(6-((4methoxybenzyl)oxy)-2-(methylsulfonyl)pyrimidin-4-yl)morpholine (180 mg, $0.48 \mathrm{mmol})$, $\left(2 R^{*}, 3 S^{*}\right)$-2-benzyl-3-methylpyrrolidine (90 mg, $\left.0.51 \mathrm{mmol}\right)$, DIPEA (166 $\left.\mu \mathrm{L}, 0.95 \mathrm{mmol}\right)$ and TBAF hydrate $(249 \mathrm{mg}, 0.95 \mathrm{mmol})$ in DMSO $(0.95 \mathrm{~mL})$ was stirred at $80{ }^{\circ} \mathrm{C}$ for $17 \mathrm{~h}$.

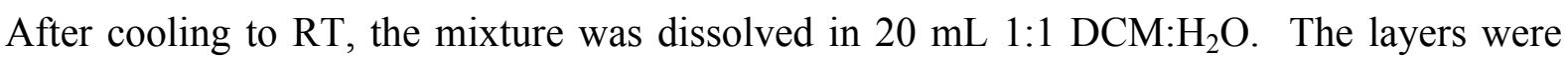
separated, the organic layer dried by passage through a hydrophobic frit and concentrated. The residue was used without further purification in the next step.

Step 6: 2-((2S,3R)-2-Benzyl-3-methylpyrrolidin-1-yl)-6-morpholinopyrimidin-4(3H)-one (68).

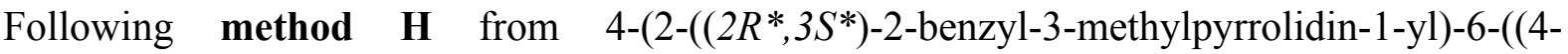


methoxybenzyl)oxy)pyrimidin-4-yl)morpholine (crude material from previous step), TFA (1.9 $\mathrm{mL})$ and DCM (1.9 mL). Purification by silica gel chromatography (gradient elution, 2-20\% $\mathrm{MeOH} / \mathrm{EtOAc}$ ), followed by chiral SFC, gave the title compound (23 mg, 13\% over two steps) as a white solid, plus its enantiomer. Absolute configuration was assigned following determination of biological activity, the $(2 S)$ isomer being the more active. LCMS $\left(\mathrm{ES}^{+}\right) 355$ $(\mathrm{M}+\mathrm{H})^{+}$, RT $3.18 \mathrm{~min}$ (Analytical Method B), RT $2.71 \mathrm{~min}$ (Analytical Method SFC4, YMC AMYLOSE-C + 0.1\% DEAISO 25\% IPA; ${ }^{1} \mathrm{H}$ NMR (400 MHz, DMSO-d $\left.{ }_{6}\right): \delta 10.22(1 \mathrm{H}, \mathrm{br}$ s), $7.31(2 \mathrm{H}, \mathrm{dd}, \mathrm{J}=7.4,7.4 \mathrm{~Hz}), 7.20(3 \mathrm{H}, \mathrm{dd}, \mathrm{J}=7.0,13.2 \mathrm{~Hz}), 4.81(1 \mathrm{H}, \mathrm{s}), 3.93(1 \mathrm{H}, \mathrm{br} \mathrm{d}$, $\mathrm{J}=7.9 \mathrm{~Hz}), 3.67-3.62(4 \mathrm{H}, \mathrm{m}), 3.48-3.42(6 \mathrm{H}, \mathrm{m}), 3.08(1 \mathrm{H}, \mathrm{dd}, \mathrm{J}=2.6,13.1 \mathrm{~Hz}), 2.68(1 \mathrm{H}$, dd, J=9.2, $12.9 \mathrm{~Hz}), 2.08-1.94(2 \mathrm{H}, \mathrm{m}), 1.51-1.44(1 \mathrm{H}, \mathrm{m}), 0.80(3 \mathrm{H}, \mathrm{d}, \mathrm{J}=6.8 \mathrm{~Hz})$. HRMS $\left(\mathrm{ESI}^{+}\right)(\mathrm{M}+\mathrm{H})^{+}$: calcd for $\mathrm{C}_{20} \mathrm{H}_{27} \mathrm{~N}_{4} \mathrm{O}_{2}, 355.2134$; found, 355.2133 . 
HPLC traces for final compounds

6-(7-Methoxy-9H-thioxanthen-4-yl)-4-morpholino-1H-pyridin-2-one (7)

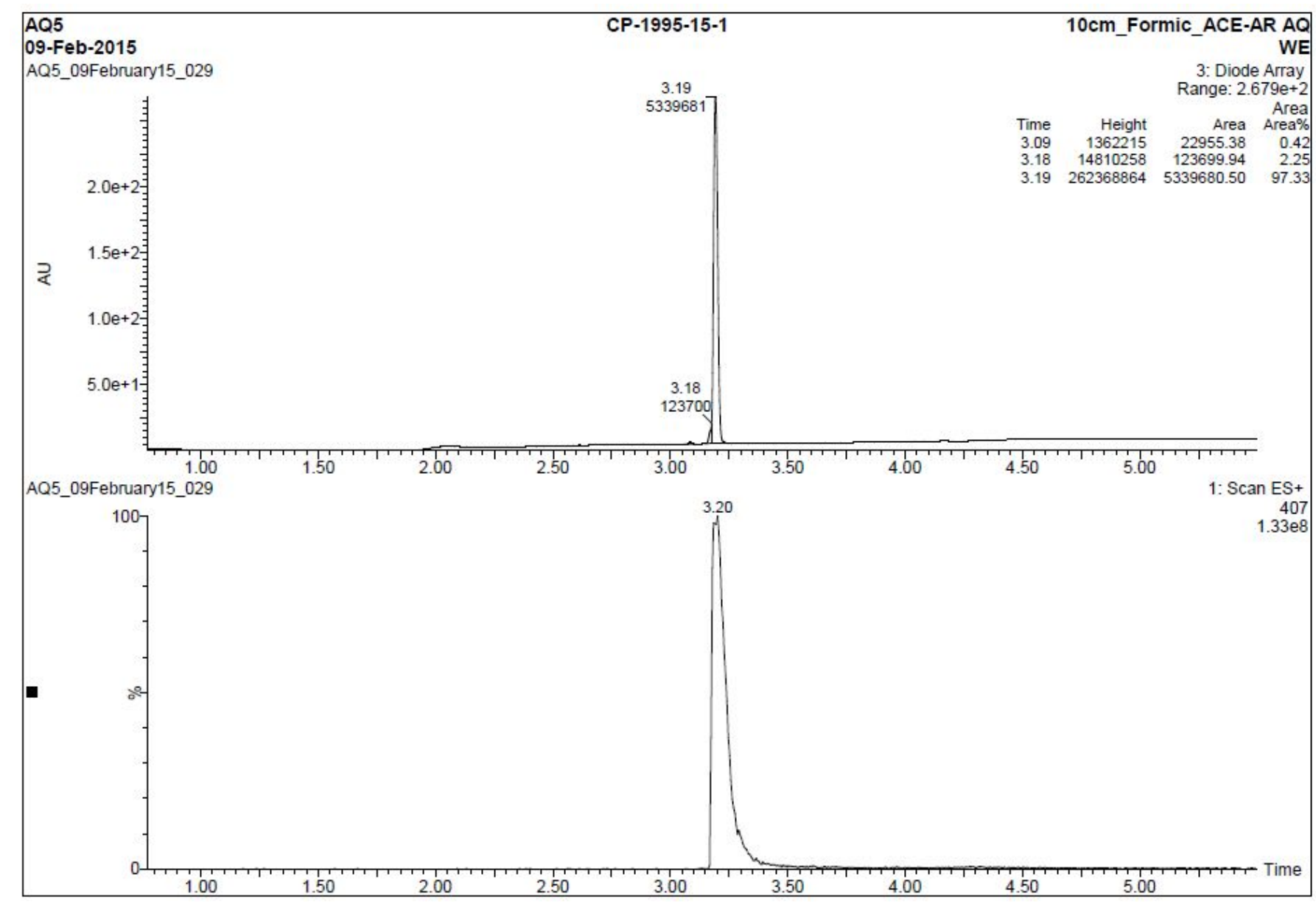


(R)-6-(7-Methoxy-9H-thioxanthen-4-yl)-4-(2-methylmorpholino)pyridin-2(1H)-one (8)

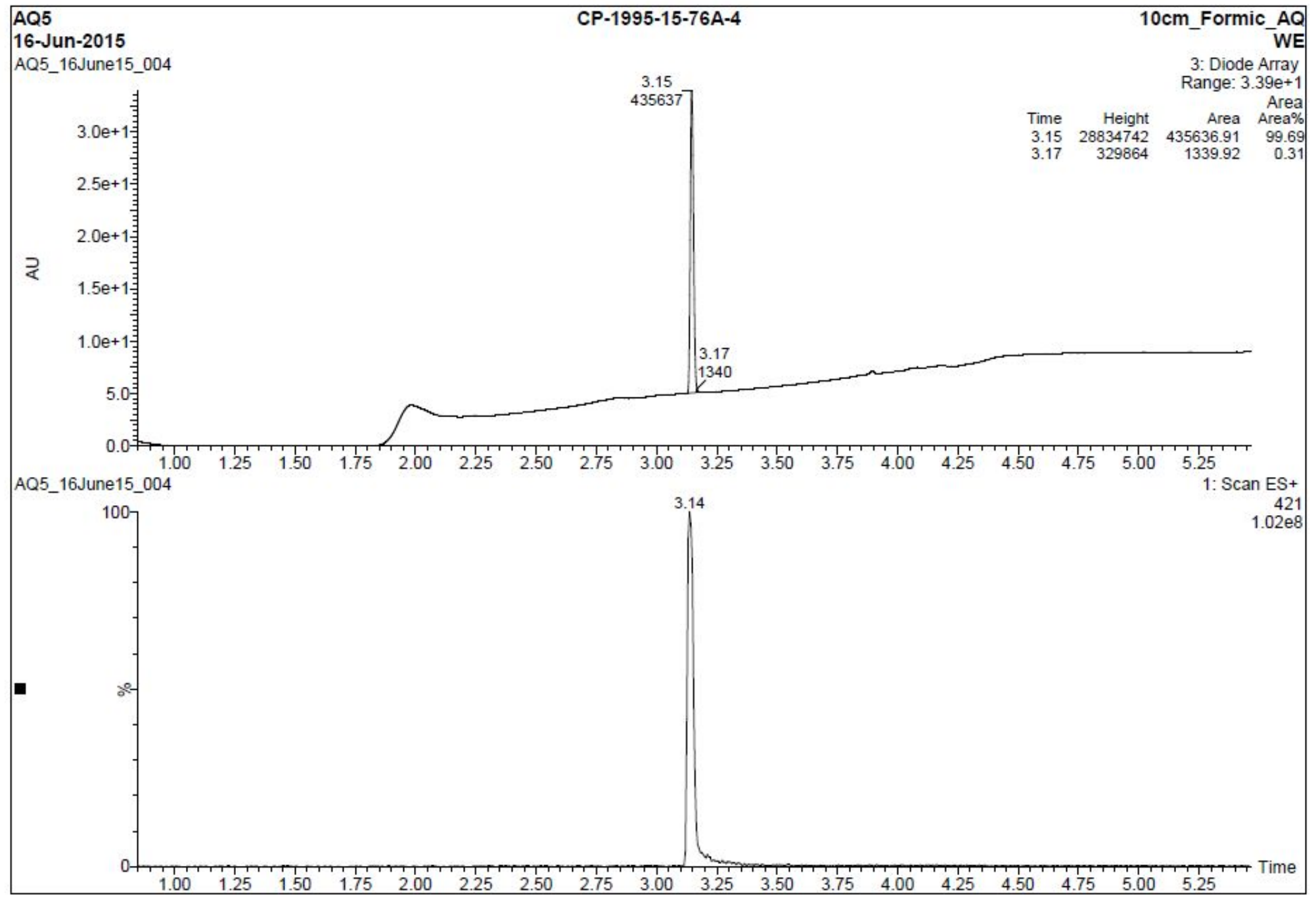

S65 
6-(7-Methoxy-9H-thioxanthen-4-yl)-1-methyl-4-morpholino-pyridin-2-one (11)

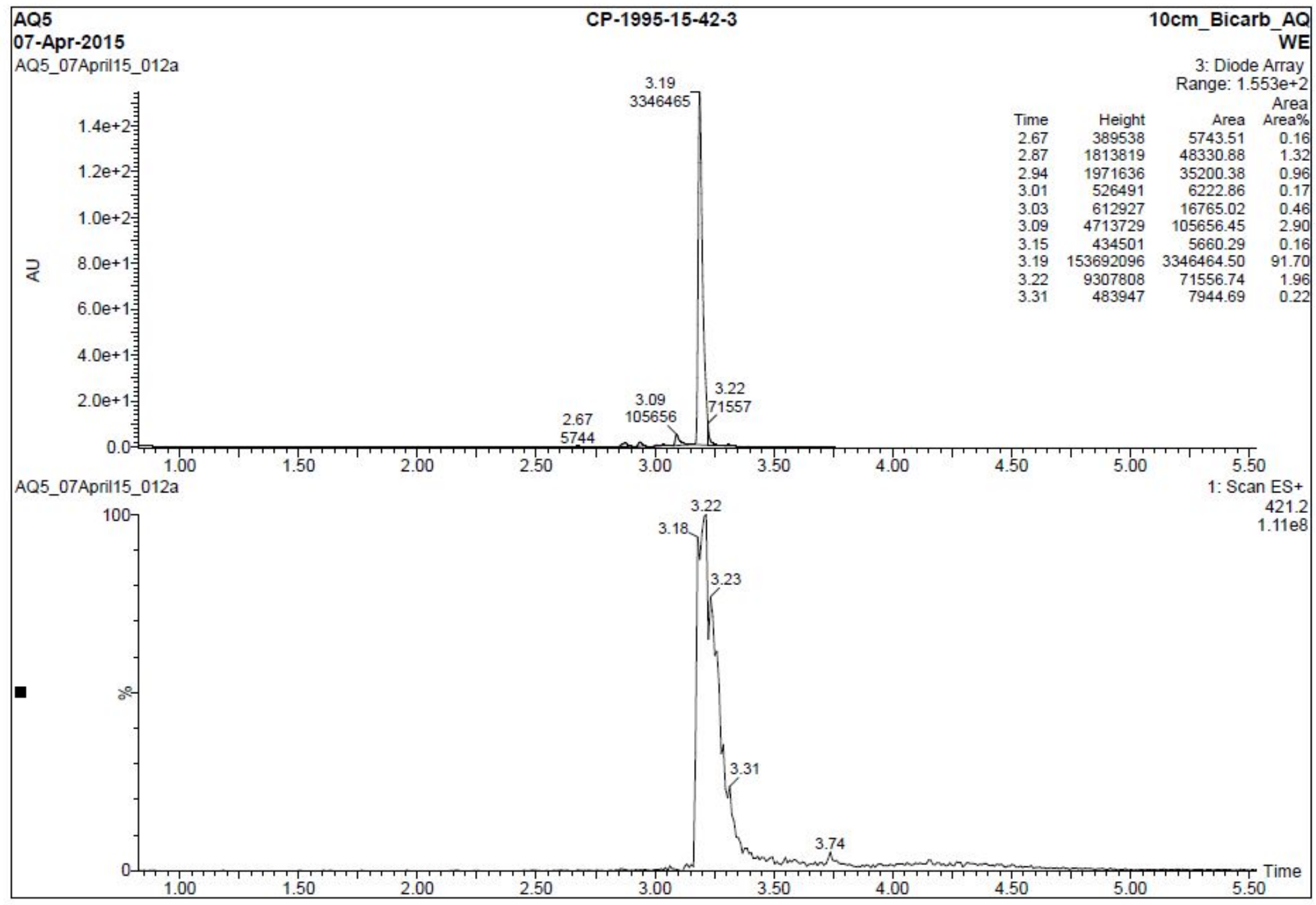


2-(7-Methoxy-9H-thioxanthen-4-yl)-6-morpholino-pyridin-4-ol (16)

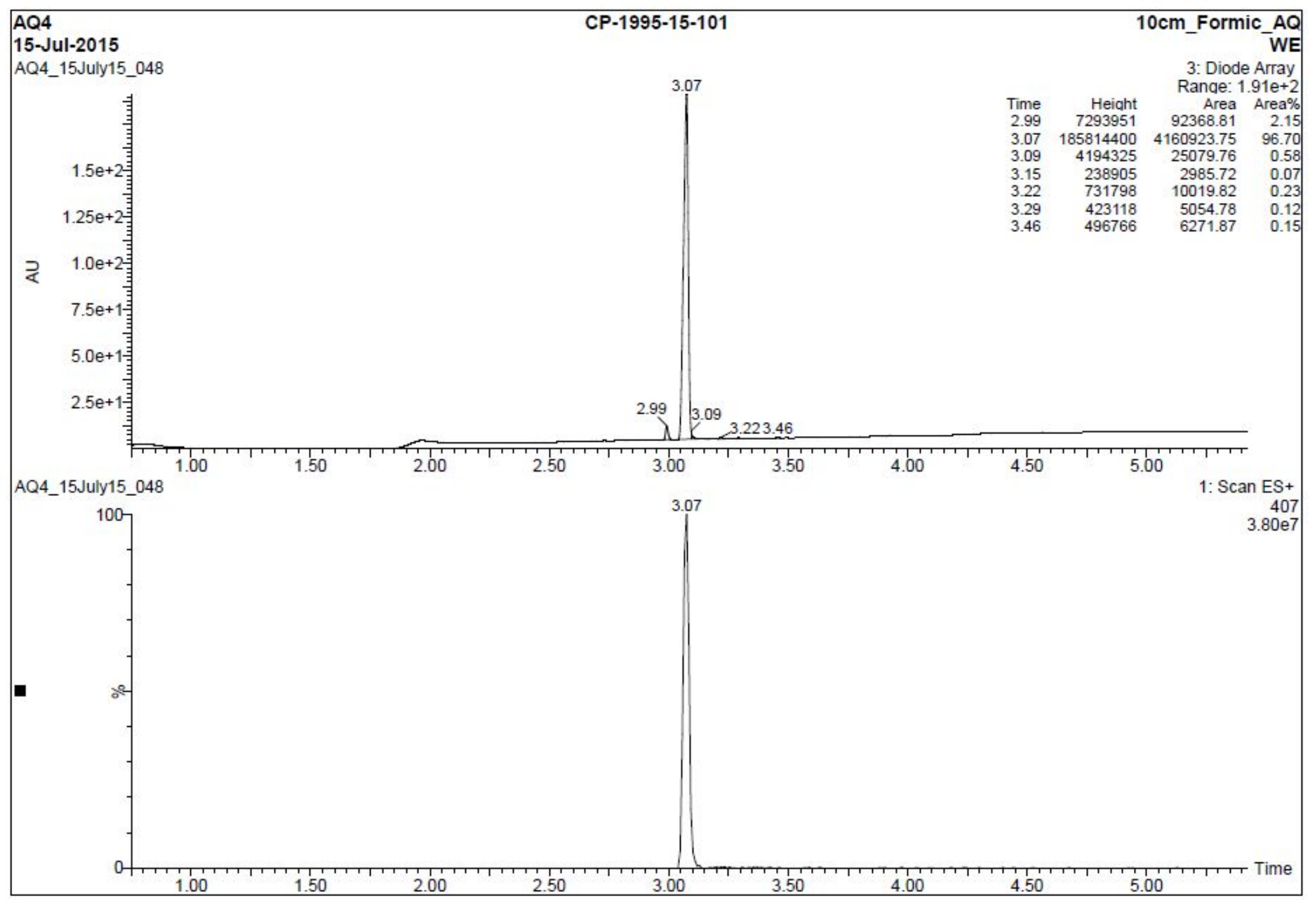


4-(7-Methoxy-9H-thioxanthen-4-yl)-6-morpholino-1H-pyridin-2-one (19)

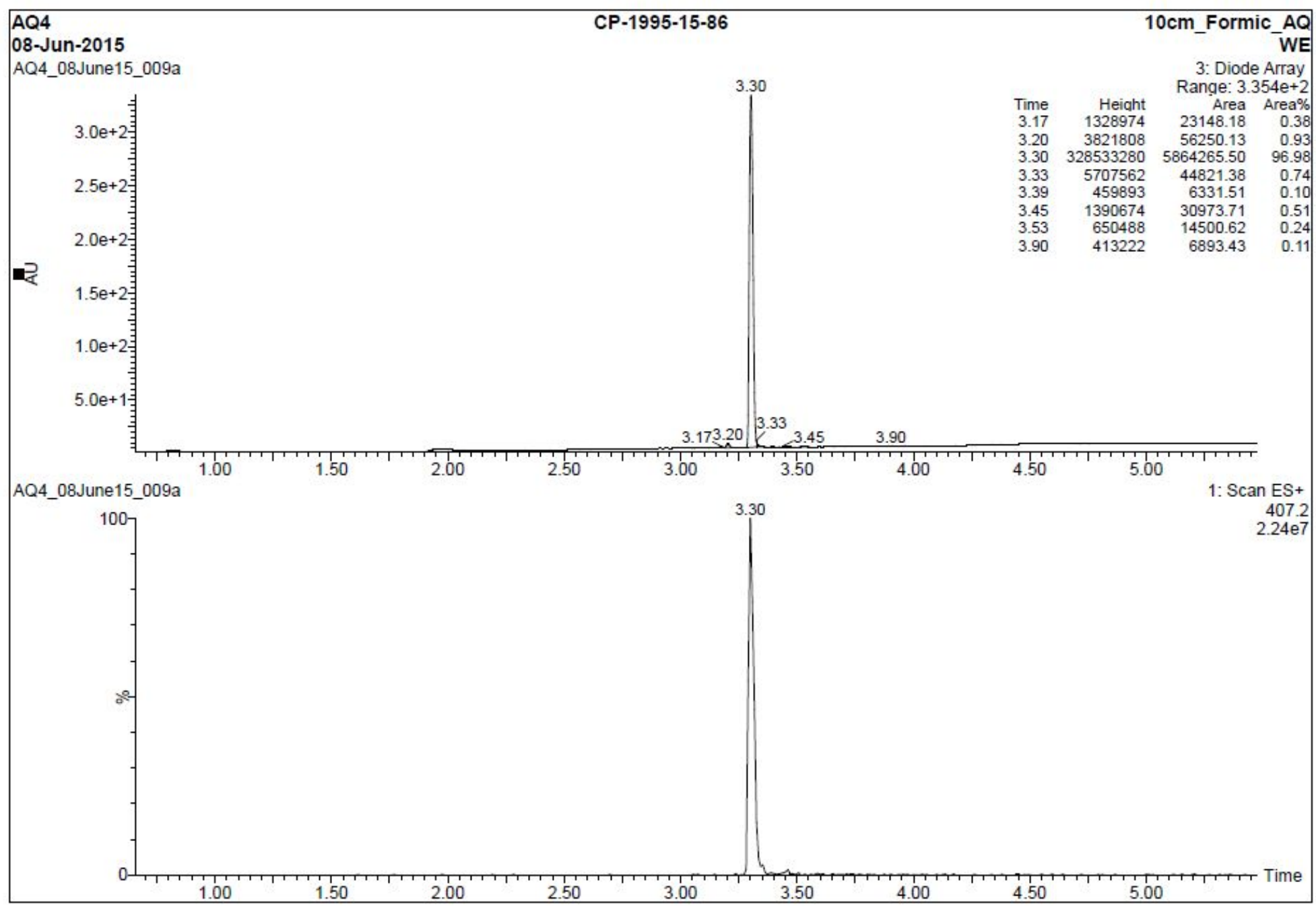


2-(7-Methoxy-9H-thioxanthen-4-yl)-6-morpholino-pyrimidin-4-ol (25)

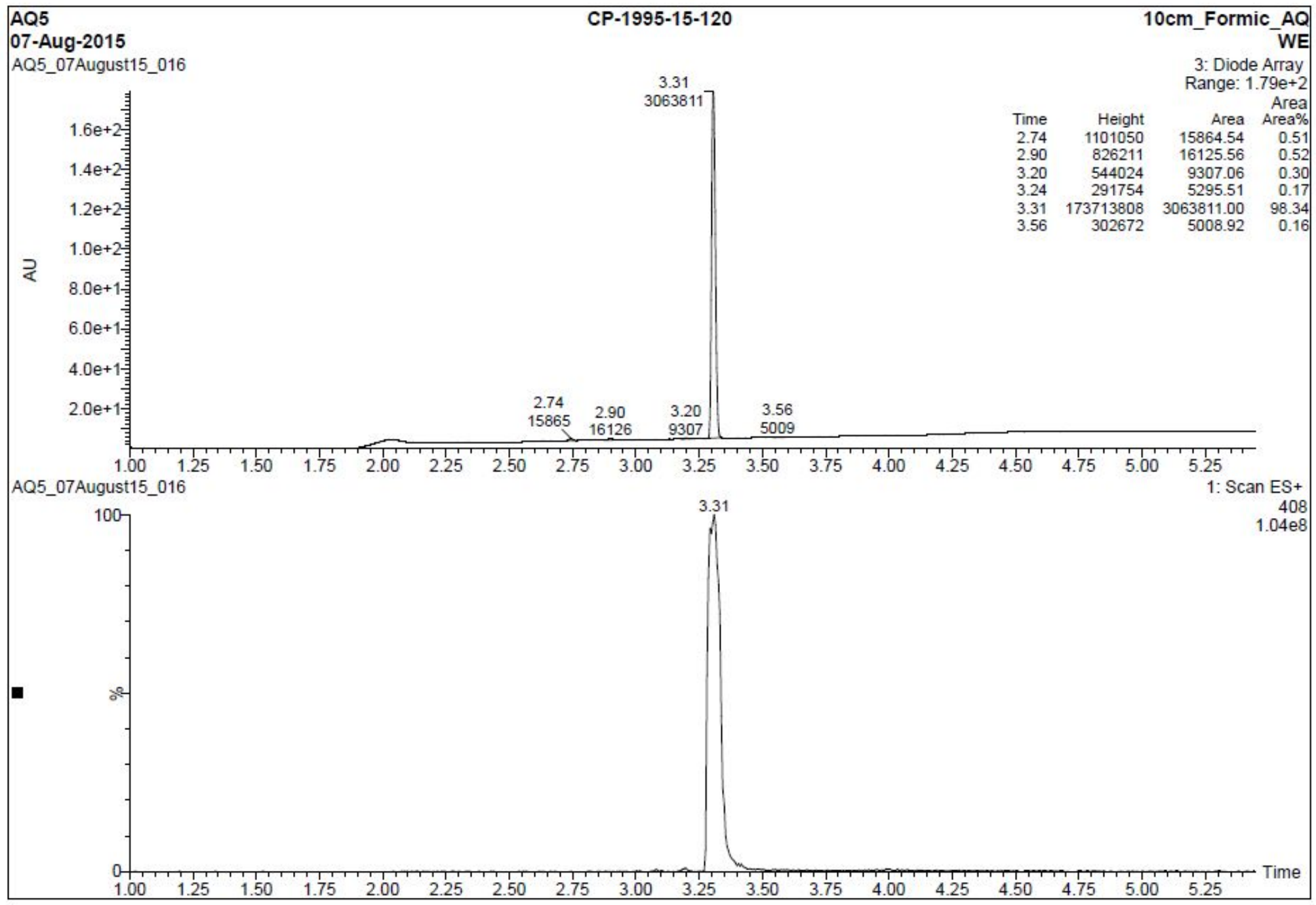


6-(7-Methoxy-9H-thioxanthen-4-yl)-2-morpholino-pyrimidin-4-ol (26)

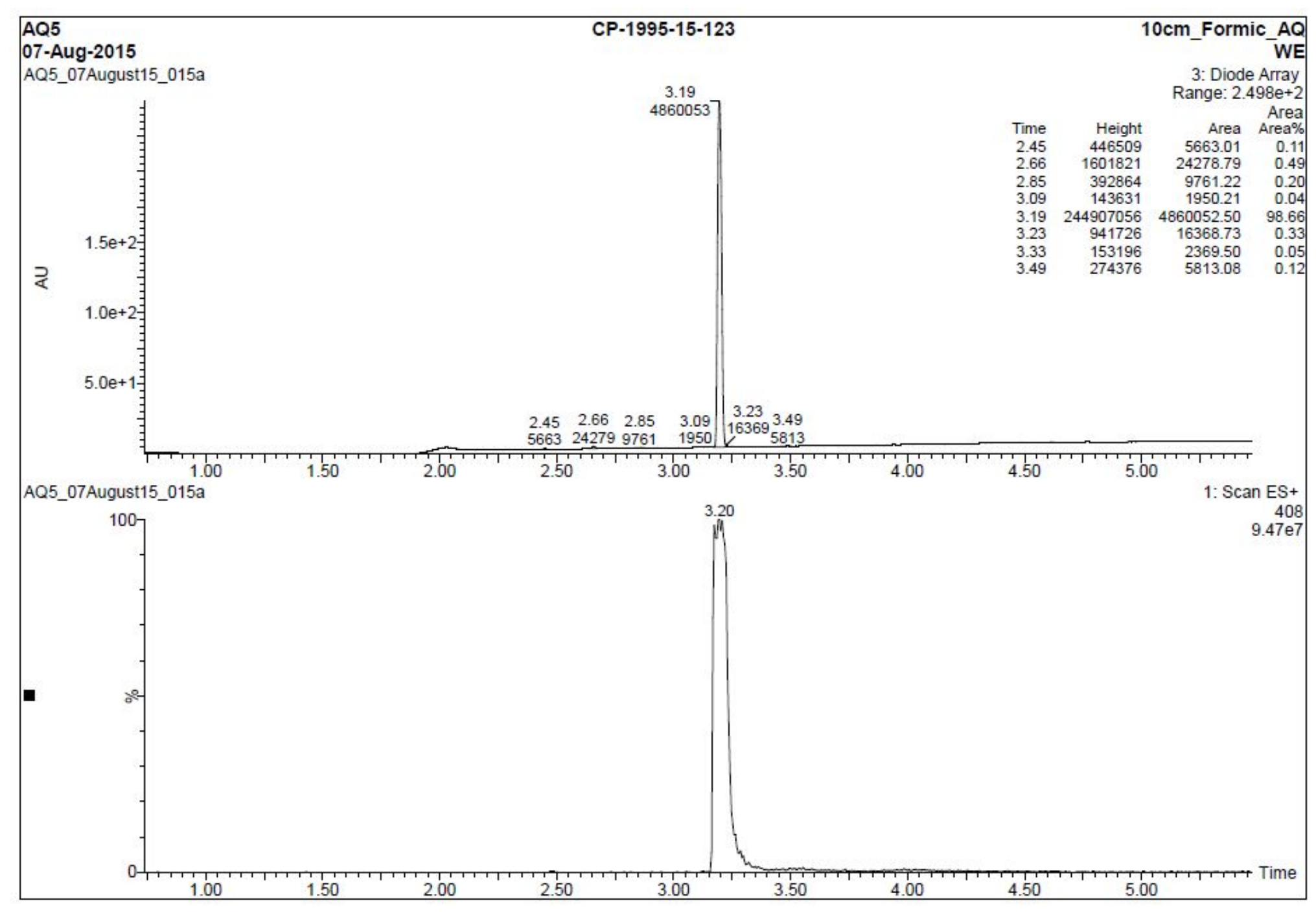


(R)-6-(2-Benzylpyrrolidin-1-yl)-2-morpholinopyrimidin-4-ol (30)

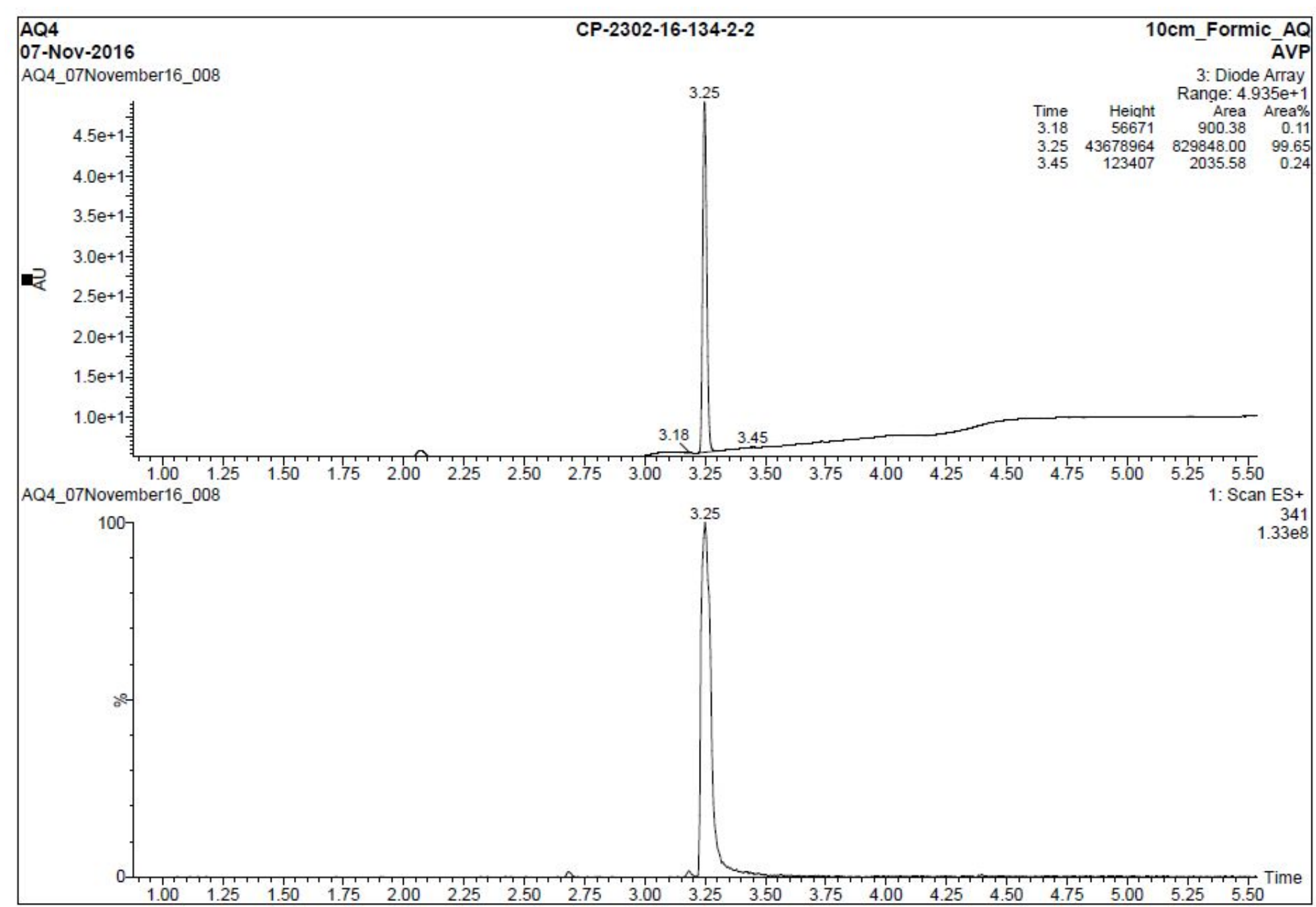


(R)-2-(2-Benzylpyrrolidin-1-yl)-6-morpholinopyrimidin-4-ol (31)

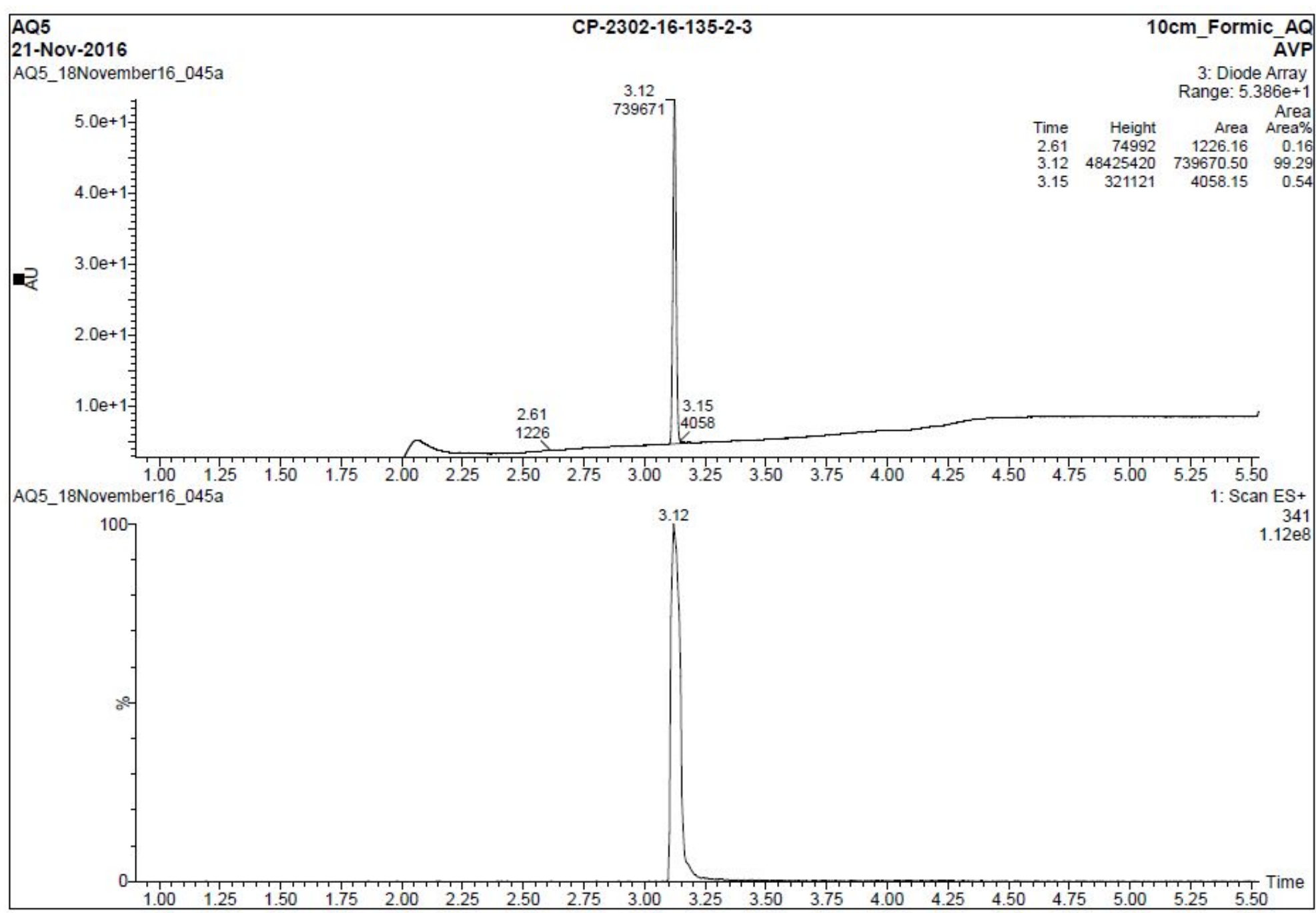


(R)-2-(2-Benzylazepan-1-yl)-6-morpholinopyrimidin-4(3H)-one (32)

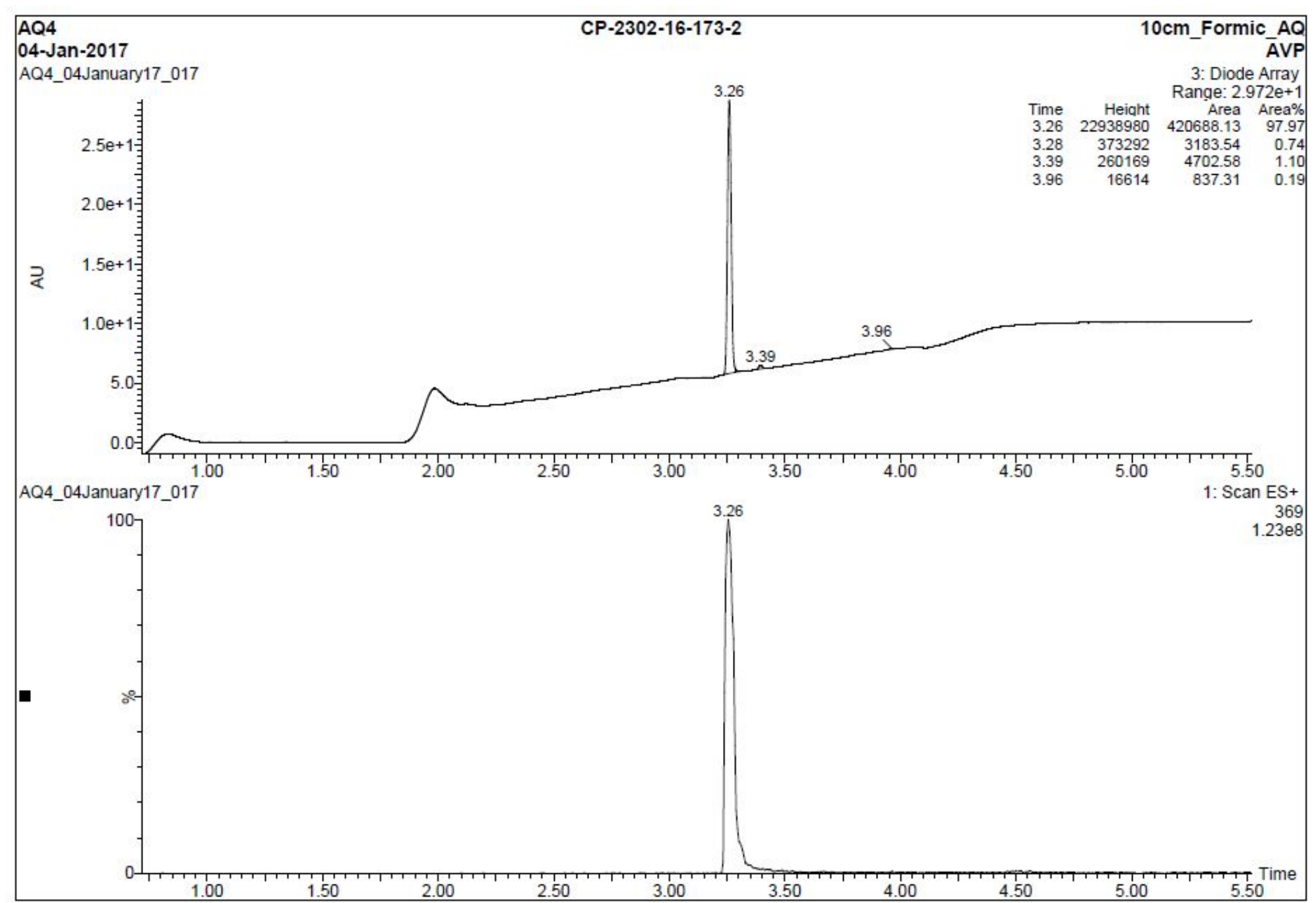


4-(7-Methoxy-9H-thioxanthen-4-yl)-6-morpholinopyrimidin-2-ol (34)

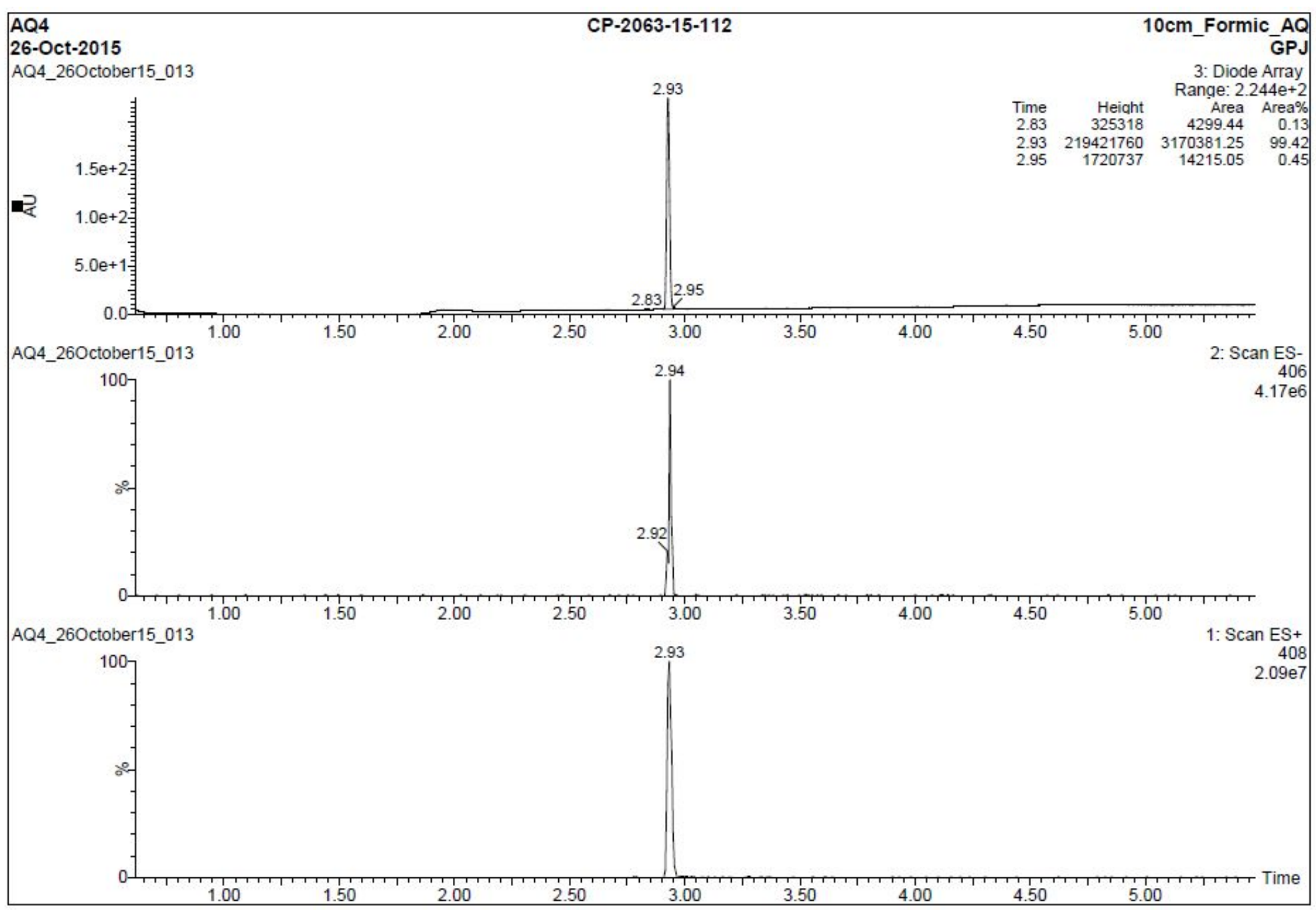


4-[2-Methoxy-6-(7-methoxy-9H-thioxanthen-4-yl)-4-pyridyl]morpholine (40)

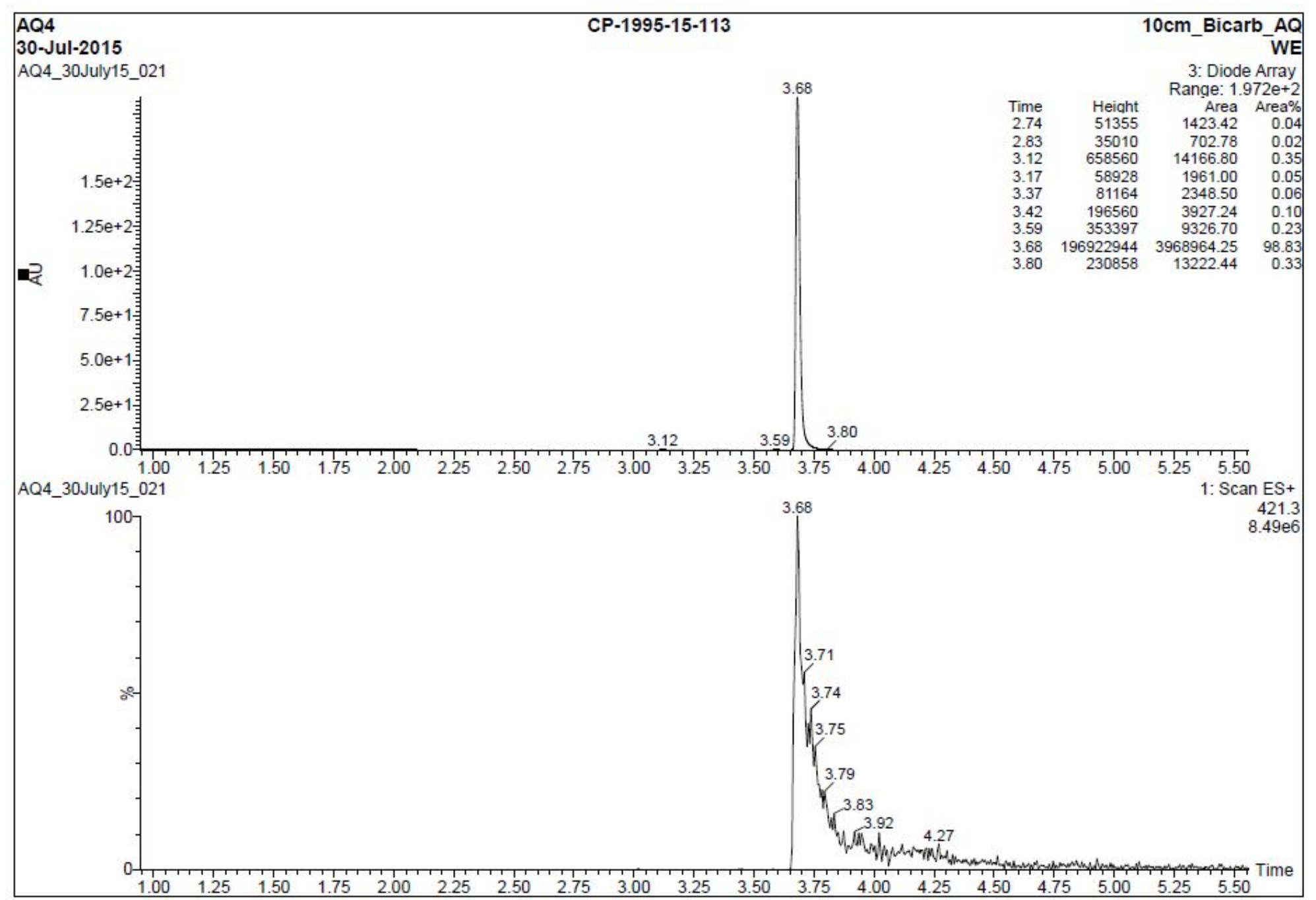


6-(2-Benzylpyrrolidin-1-yl)-4-morpholinopyridin-2(1H)-one (43)

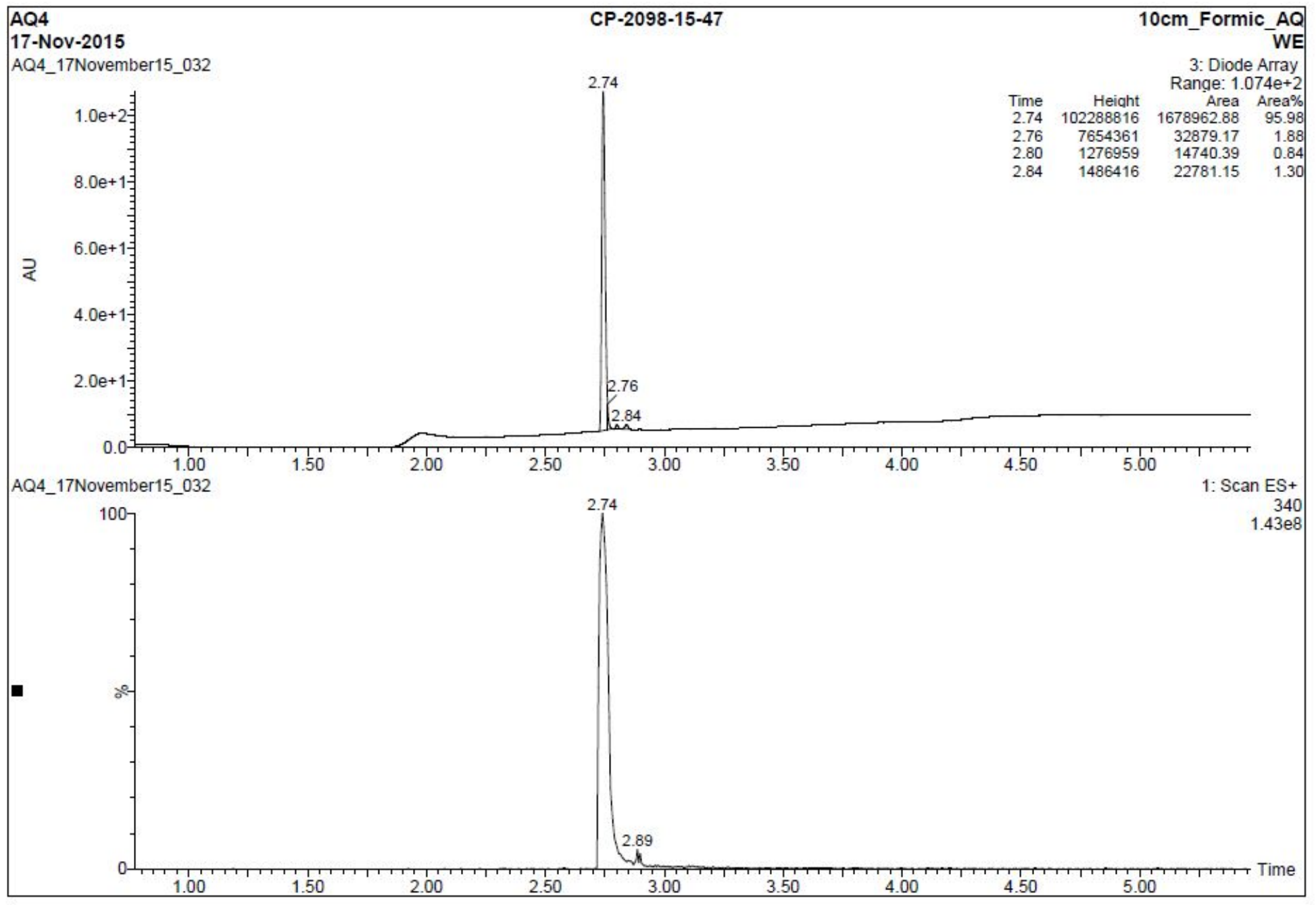


(R)-6-(2-Benzylpyrrolidin-1-yl)-4-morpholinopyridin-2(1H)-one (44)

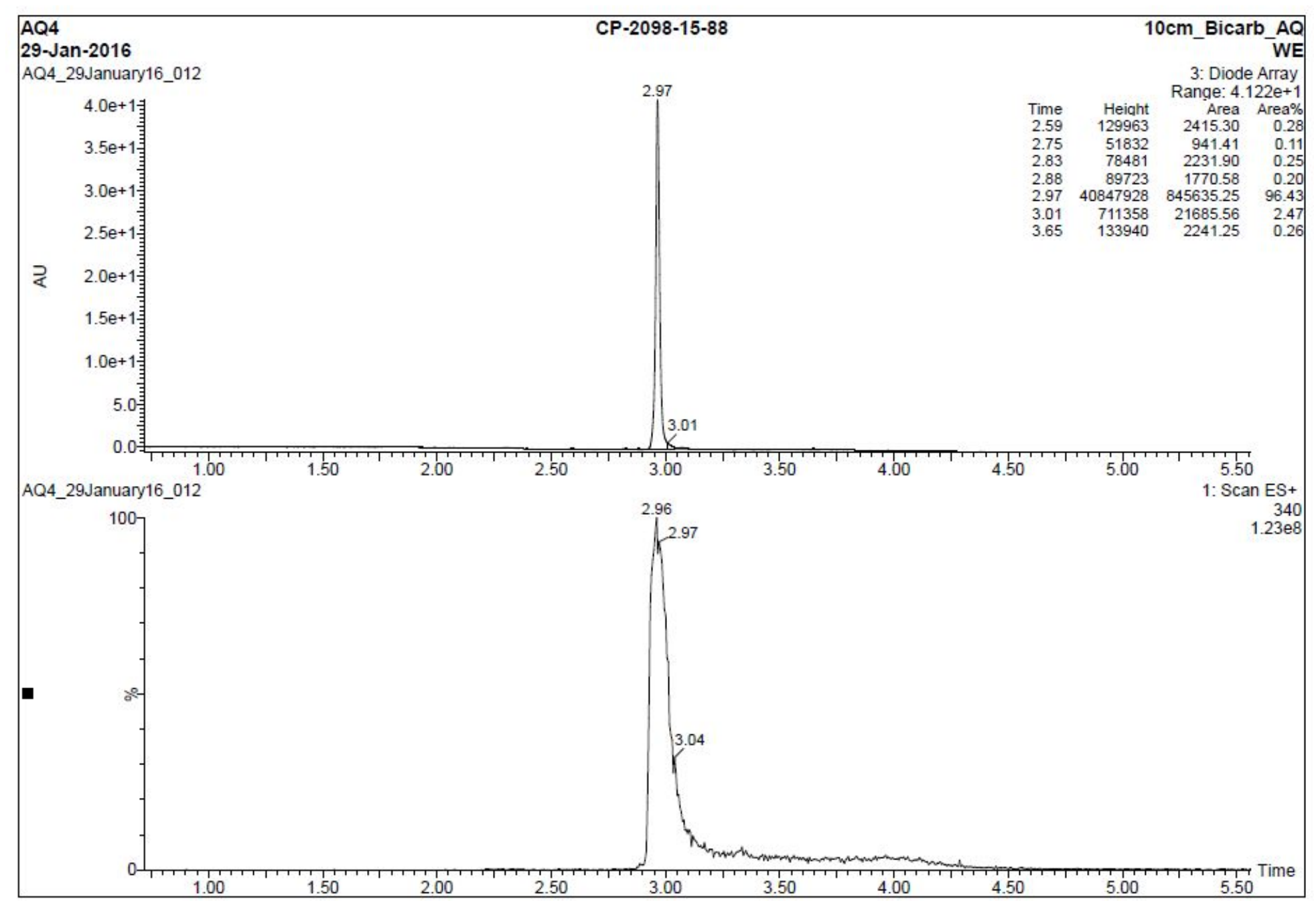


(S)-6-(2-benzylpyrrolidin-1-yl)-4-morpholinopyridin-2(1H)-one (45)

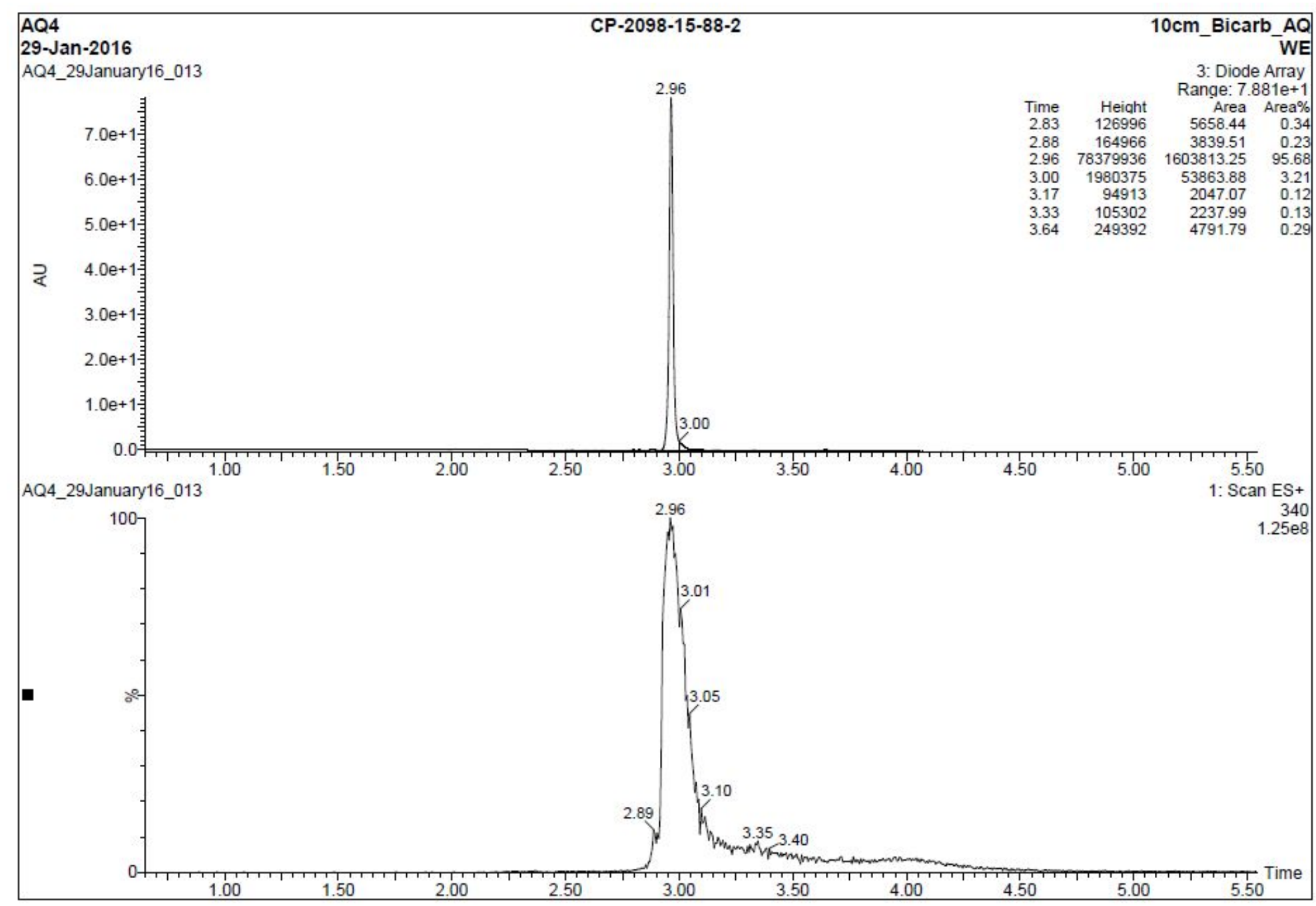


6-(3-Benzylpyrrolidin-1-yl)-4-morpholinopyridin-2(1H)-one (46)

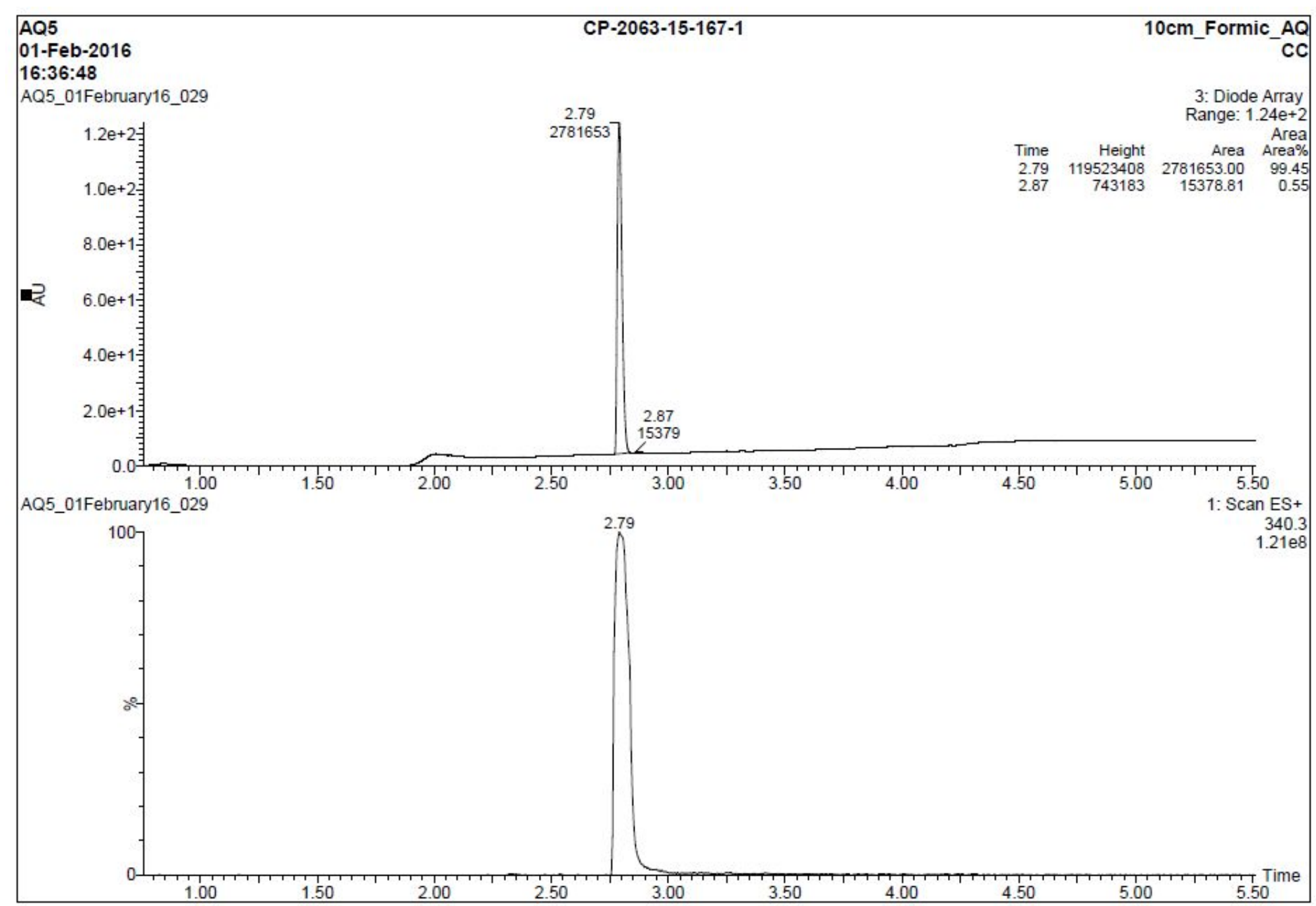


(S)-6-(2-Benzyl-3,3-difluoropyrrolidin-1-yl)-4-morpholinopyridin-2(1H)-one (47)

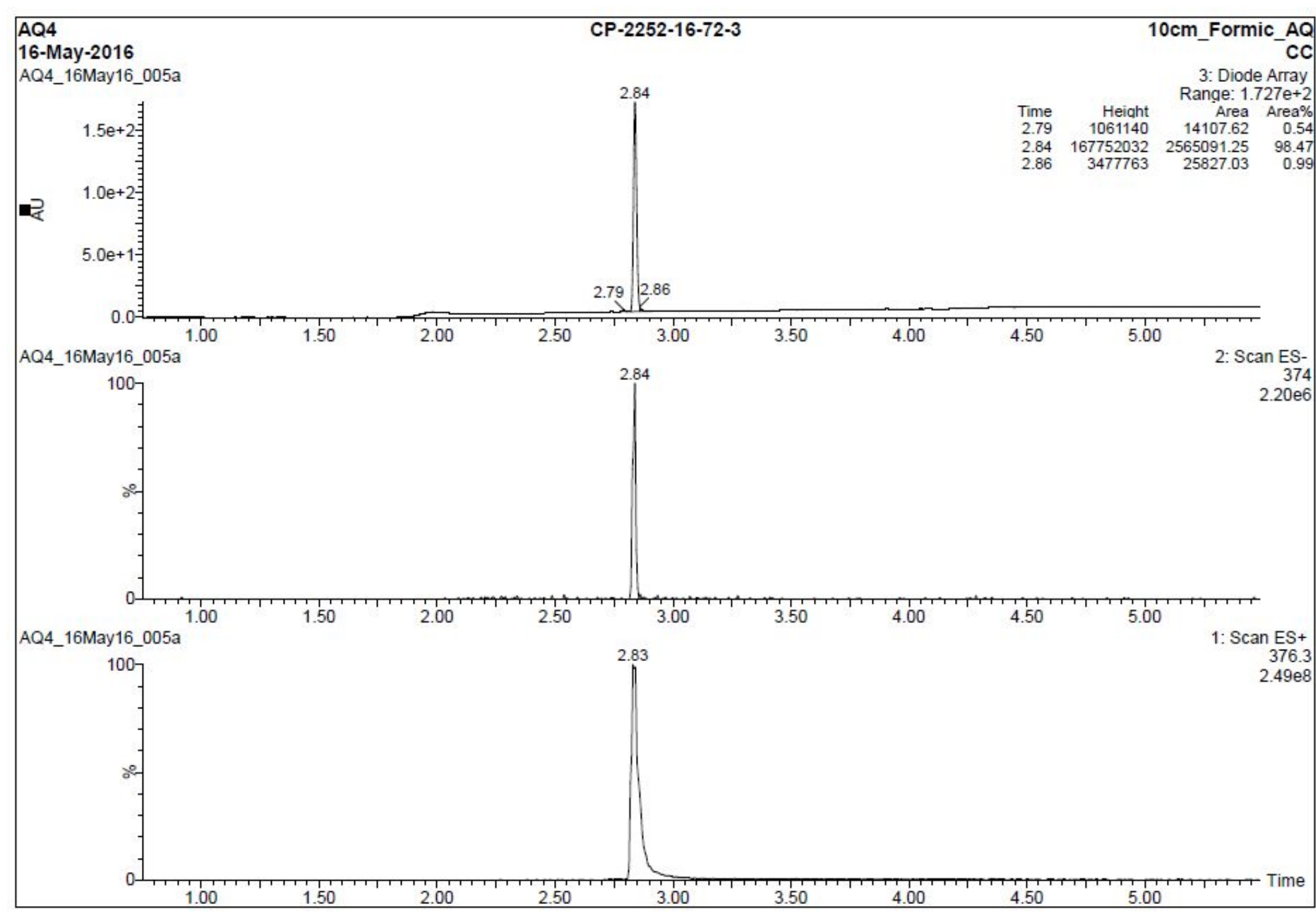


6-((2S,3R)-2-Benzyl-3-fluoropyrrolidin-1-yl)-4-morpholinopyridin-2(1H)-one (48).

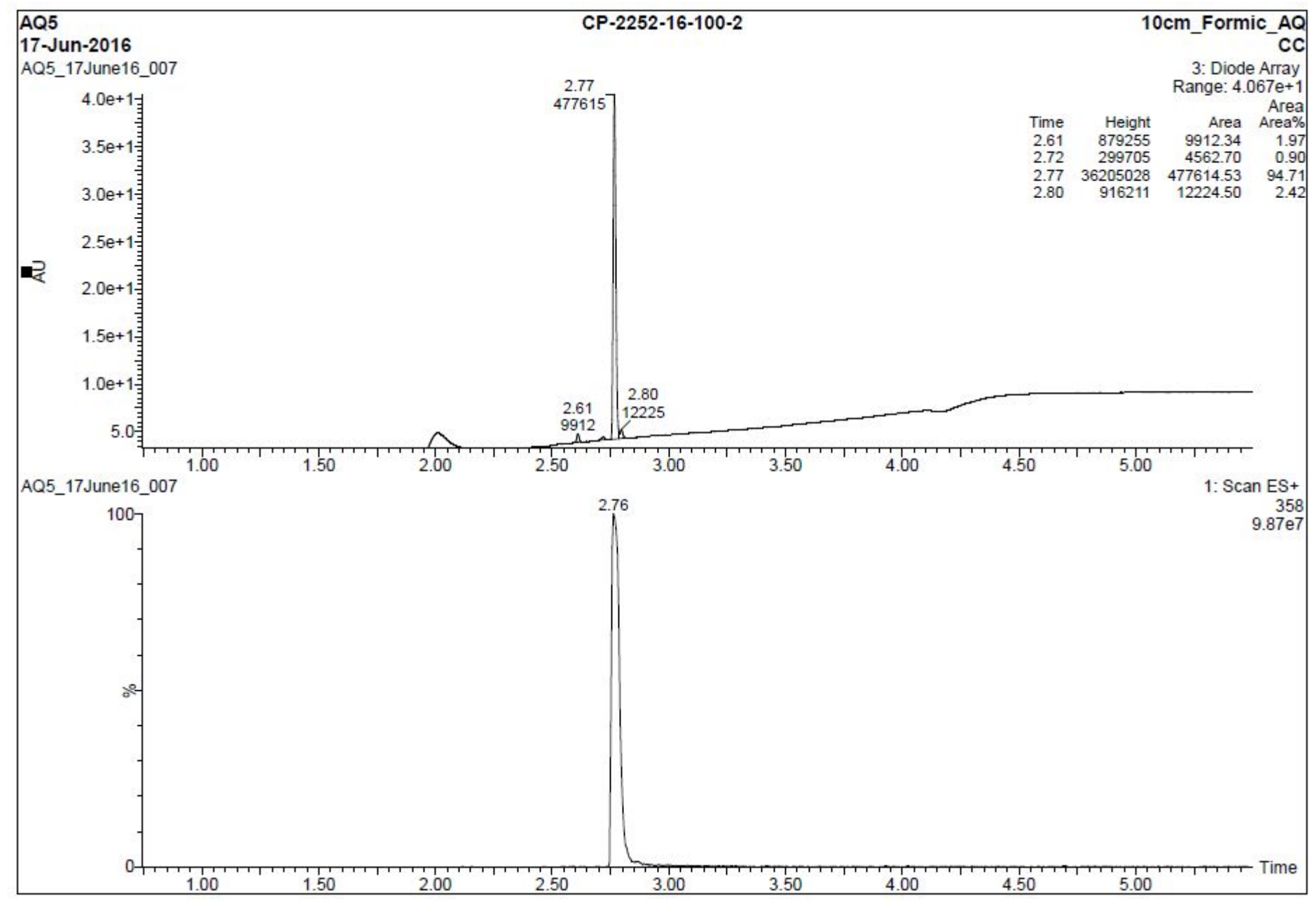


6-((2S,3R)-2-Benzyl-3-methoxypyrrolidin-1-yl)-4-morpholinopyridin-2(1H)-one (49)

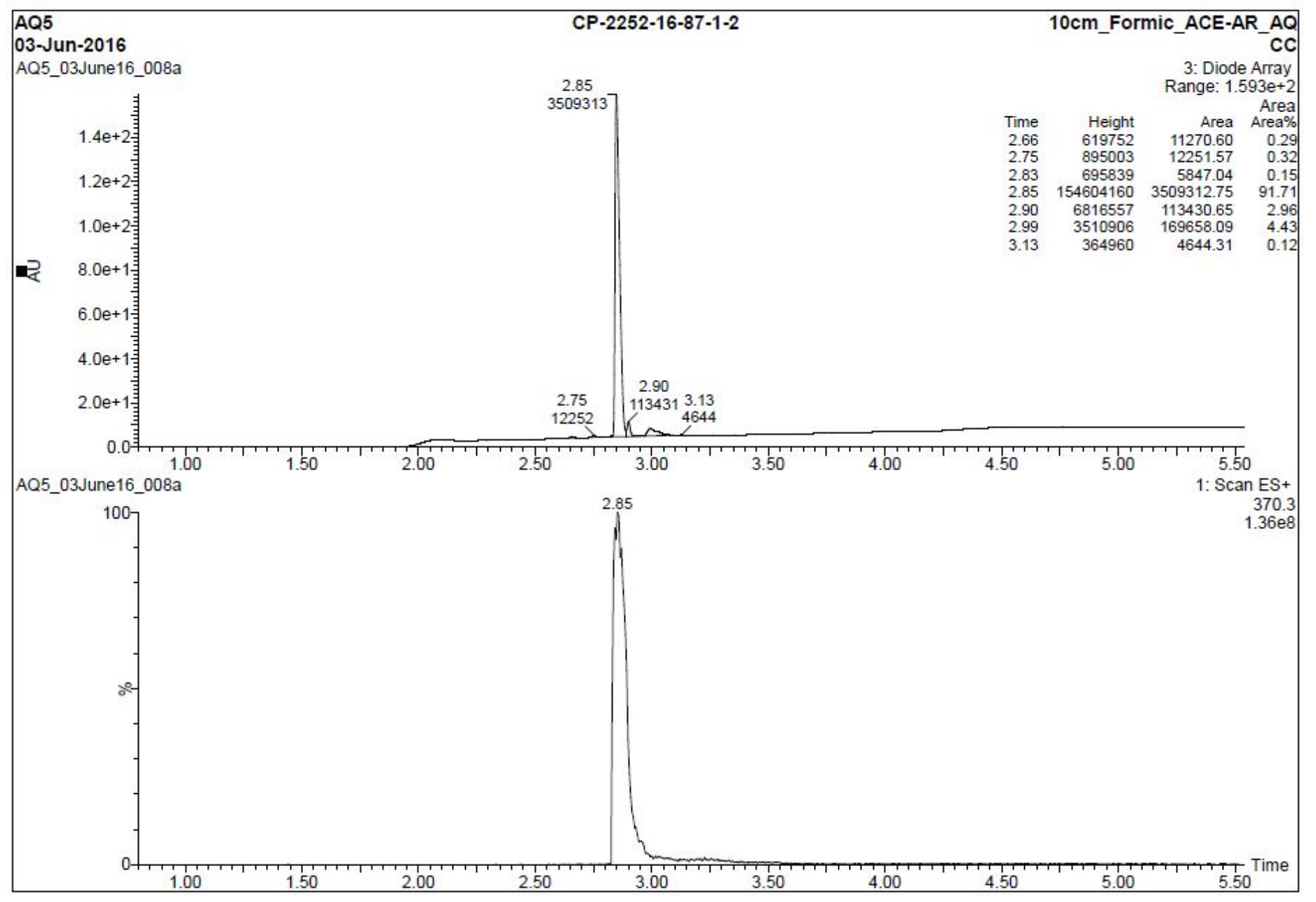


6-((1S,2S,5R)-2-Benzyl-3-azabicyclo[3.1.0]hexan-3-yl)-4-morpholinopyridin-2(1H)-one (50)

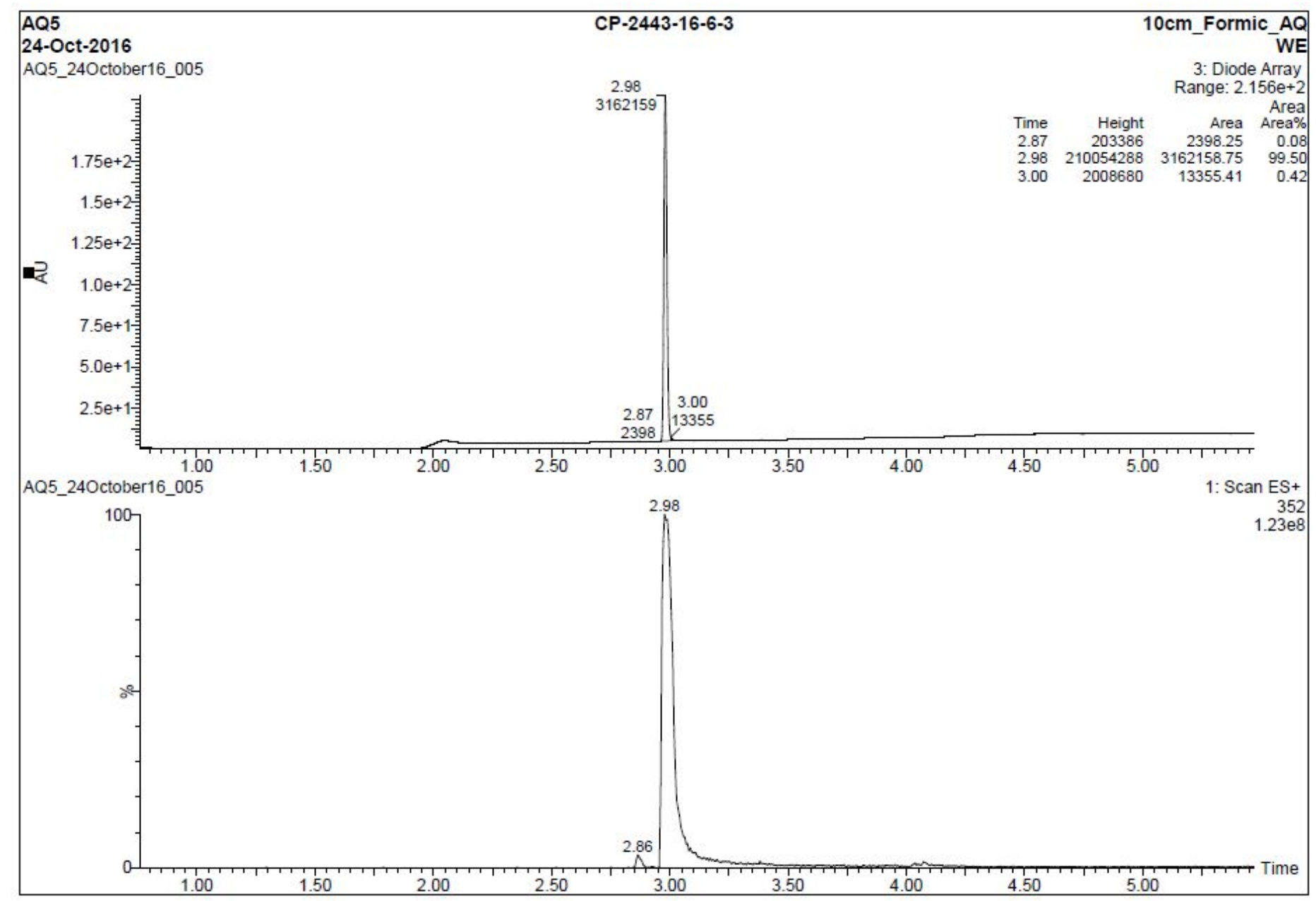


6-((1S,2S,5R)-2-Benzyl-3-azabicyclo[3.1.0]hexan-3-yl)-4-((R)-2-methylmorpholino)pyridin-2(1H)-one (51)

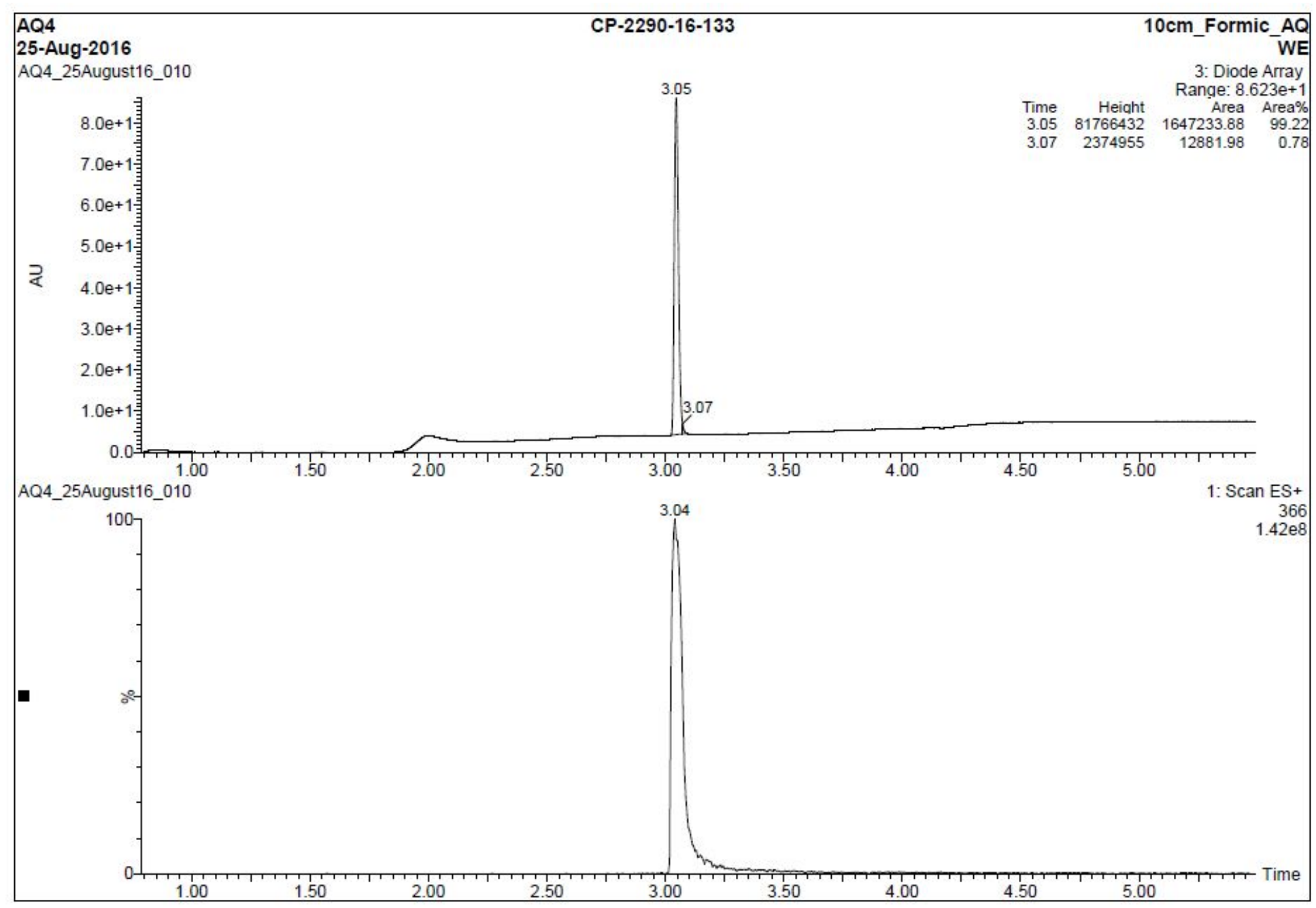


6-((1R,2S,5S)-2-Benzyl-3-azabicyclo[3.1.0]hexan-3-yl)-4-morpholinopyridin-2(1H)-one (52)

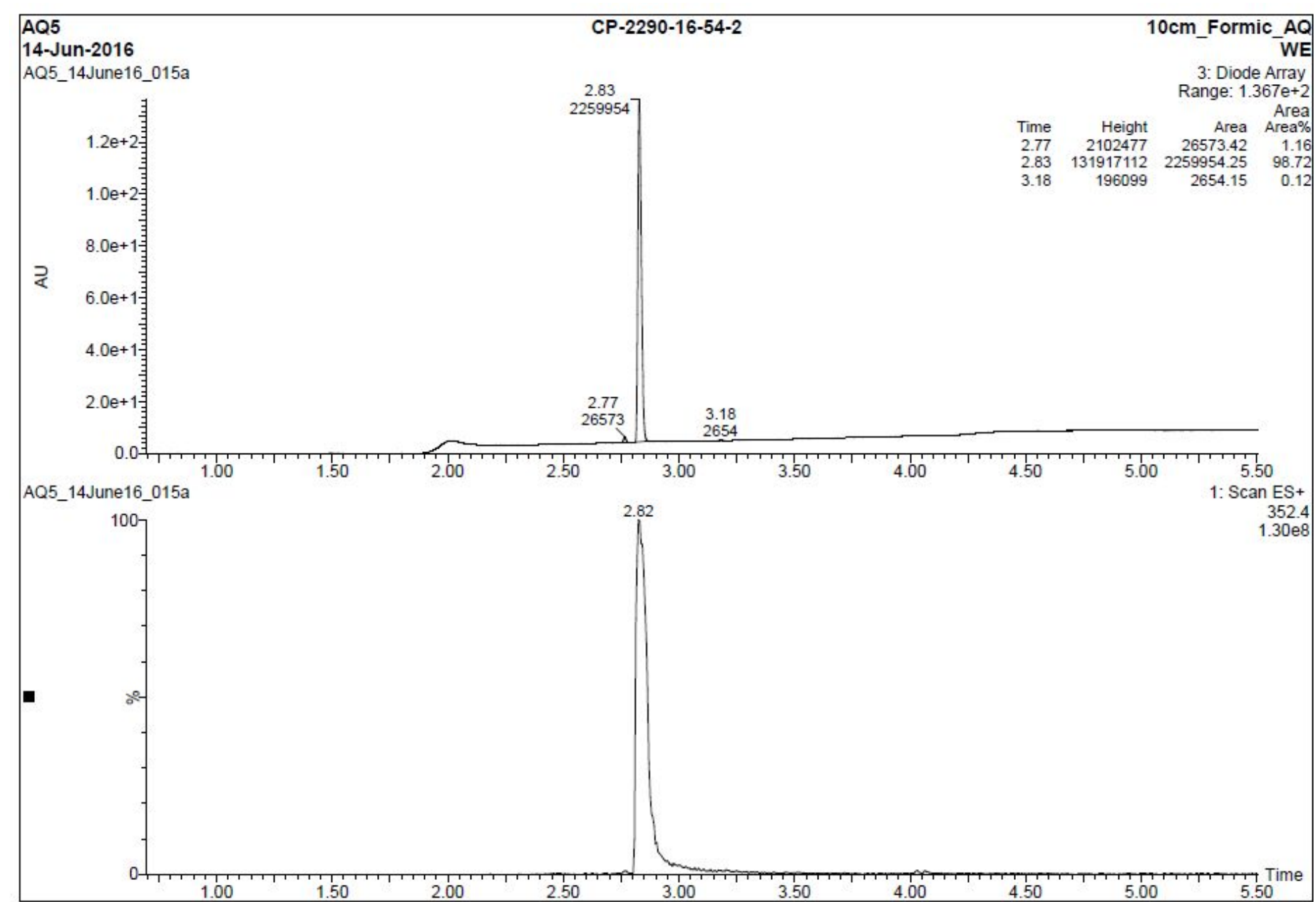


6-((1R,2S, 5S)-2-Benzyl-3-azabicyclo[3.1.0]hexan-3-yl)-4-((R)-2-methylmorpholino) pyridin-2(1H)-one (53)

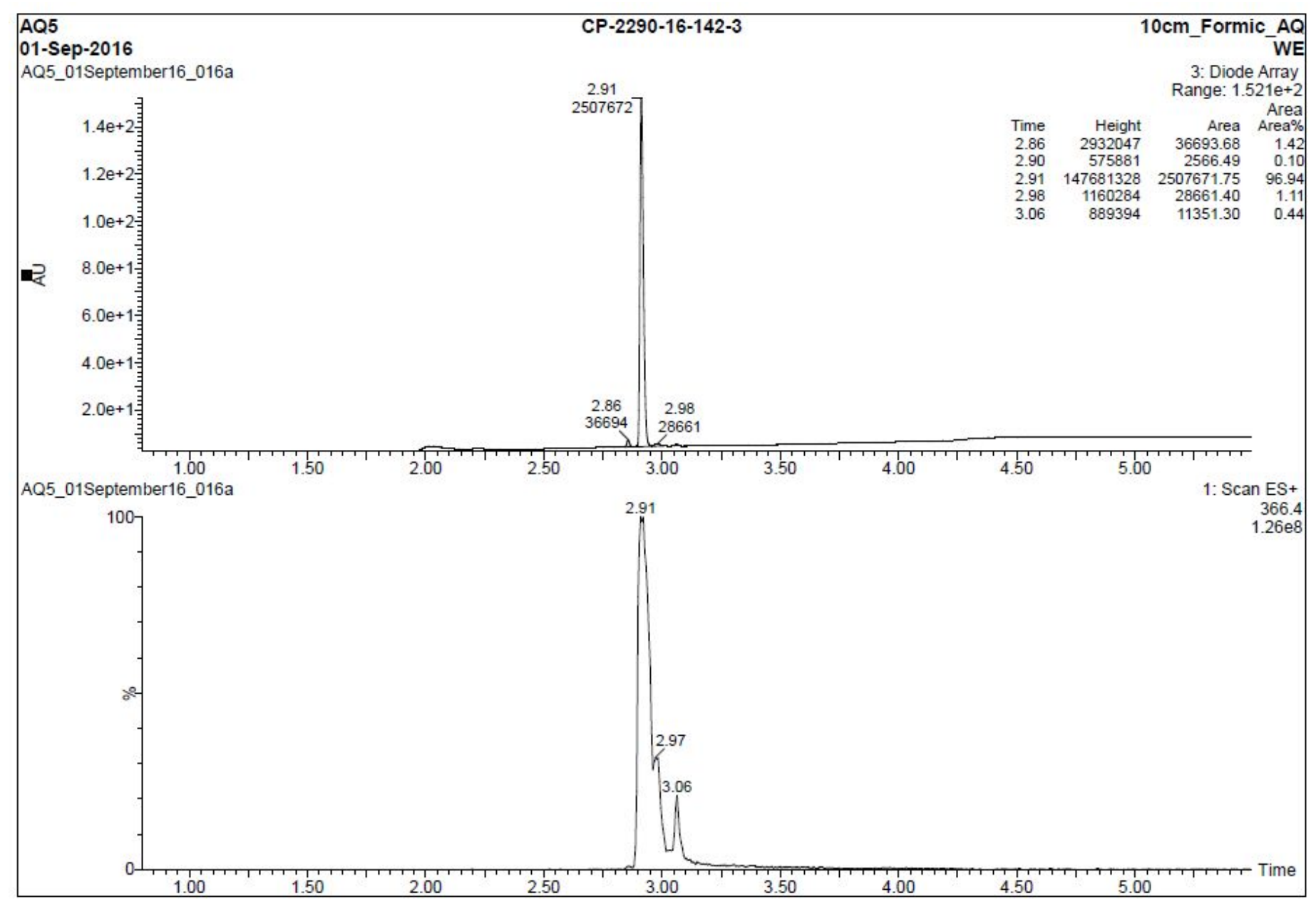


(R)-6-(2-Benzylpiperidin-1-yl)-4-morpholinopyridin-2(1H)-one (54)

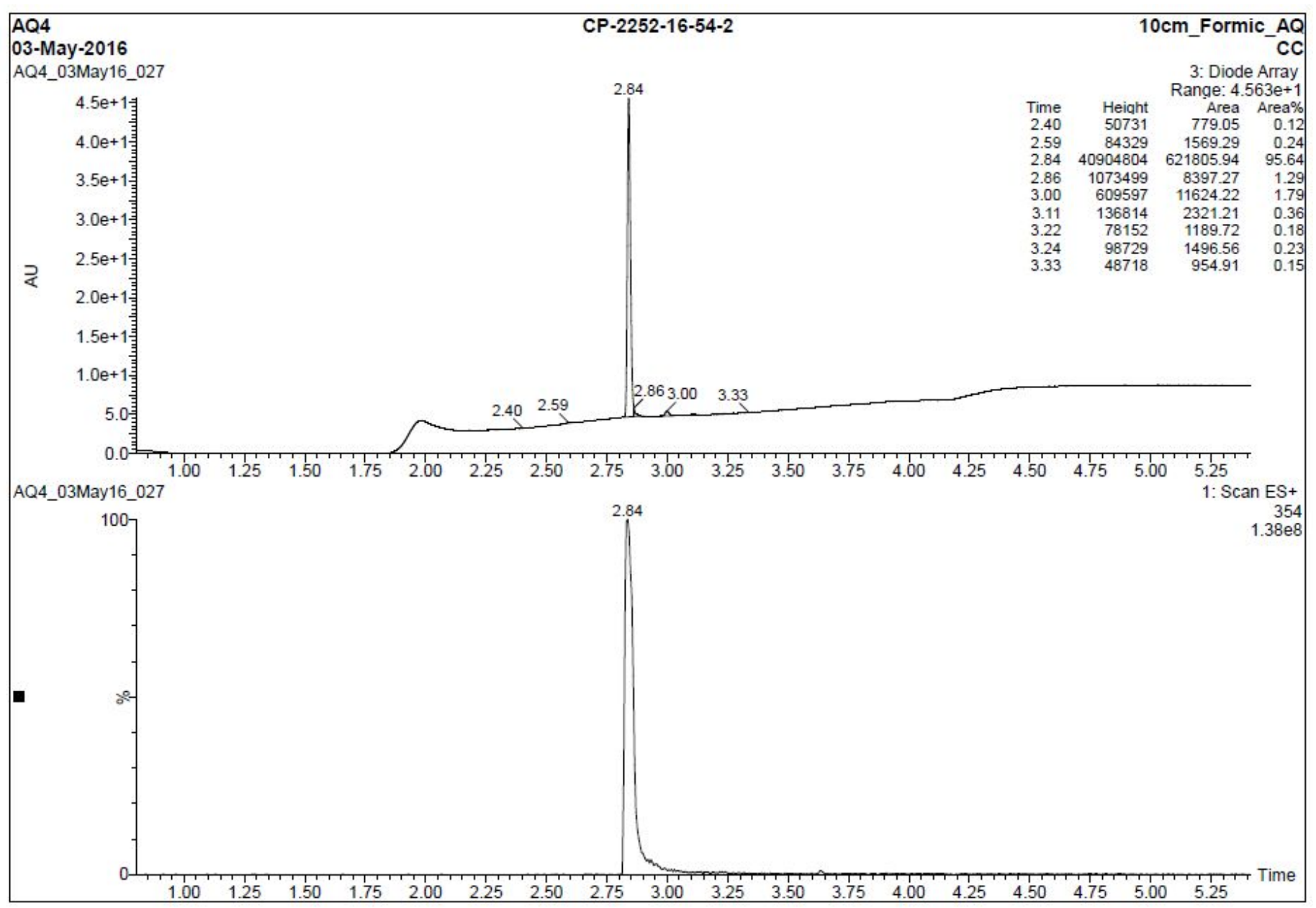


(R)-6-(2-Benzylazepan-1-yl)-4-morpholinopyridin-2(1H)-one (55)

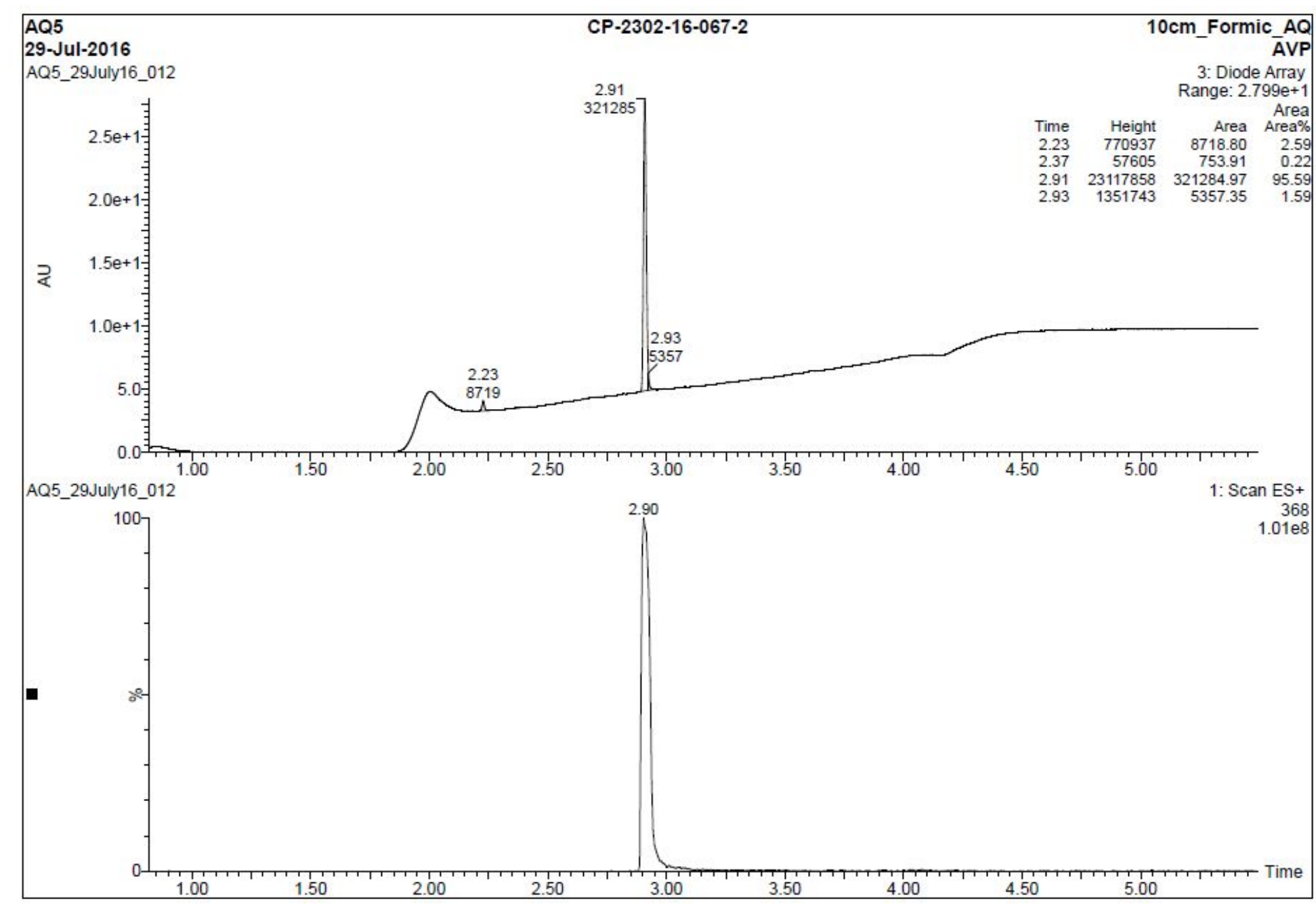


6-((R)-2-Benzylazepan-1-yl)-4-((R)-2-methylmorpholino)pyridin-2(1H)-one (56)

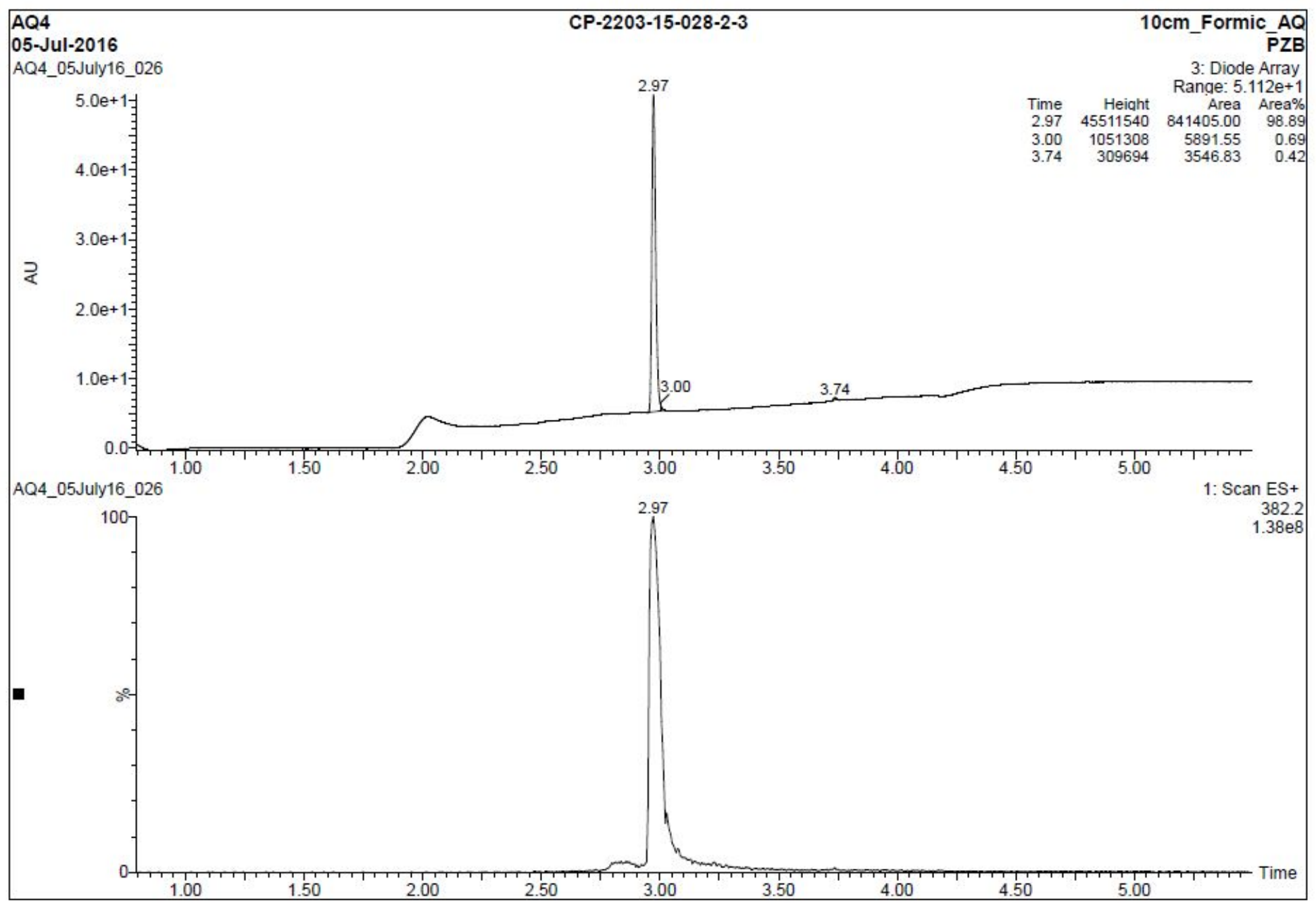


(R)-2-(2-Benzylazepan-1-yl)-6-morpholinopyrimidin-4(3H)-one (62)

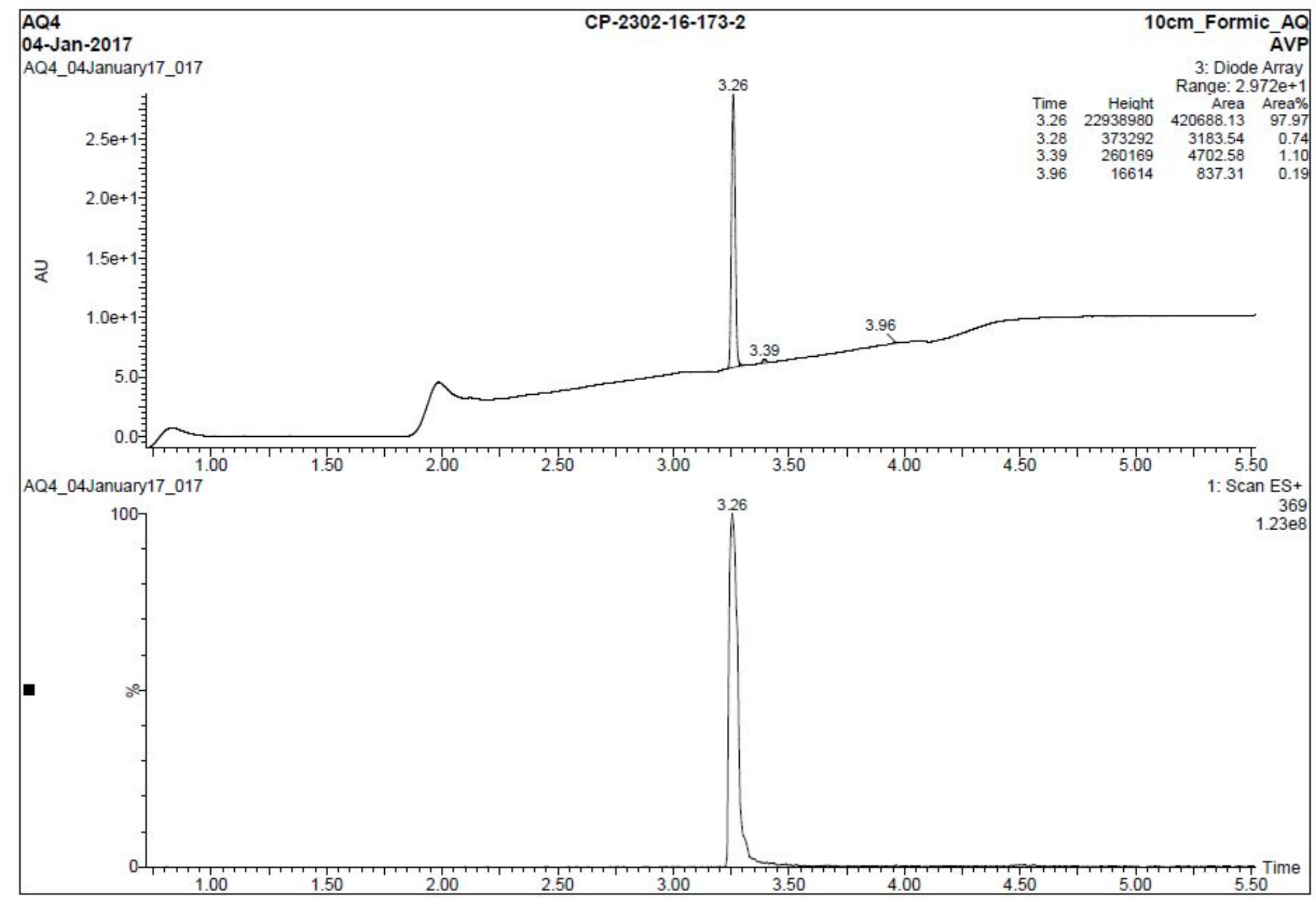


2-((R)-2-Benzylazepan-1-yl)-6-((R)-2-methylmorpholino)pyrimidin-4(3H)-one (63)

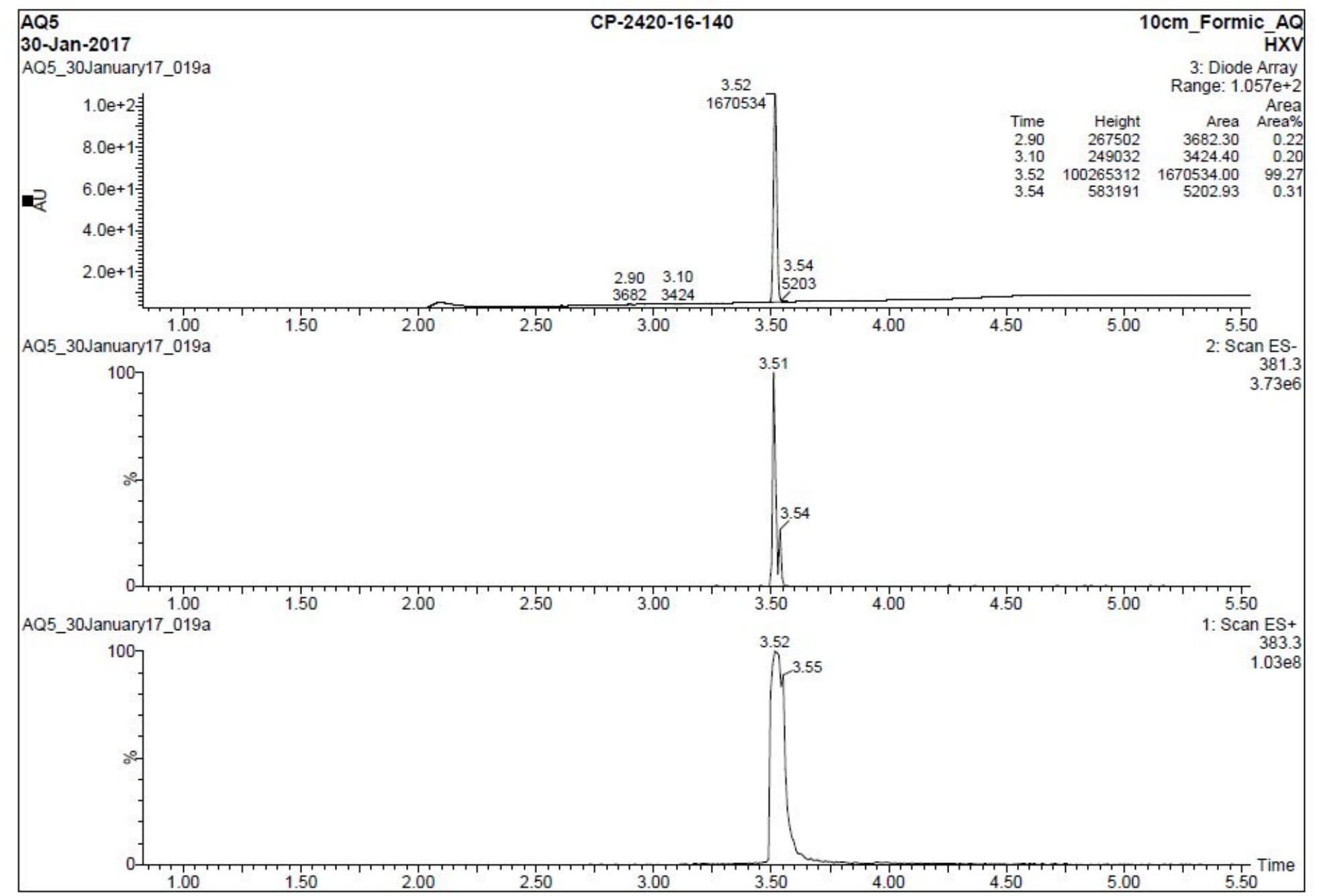


2-((1R,2S,5S)-2-Benzyl-3-azabicyclo[3.1.0]hexan-3-yl)-6-morpholinopyrimidin-4(3H)-one (64)

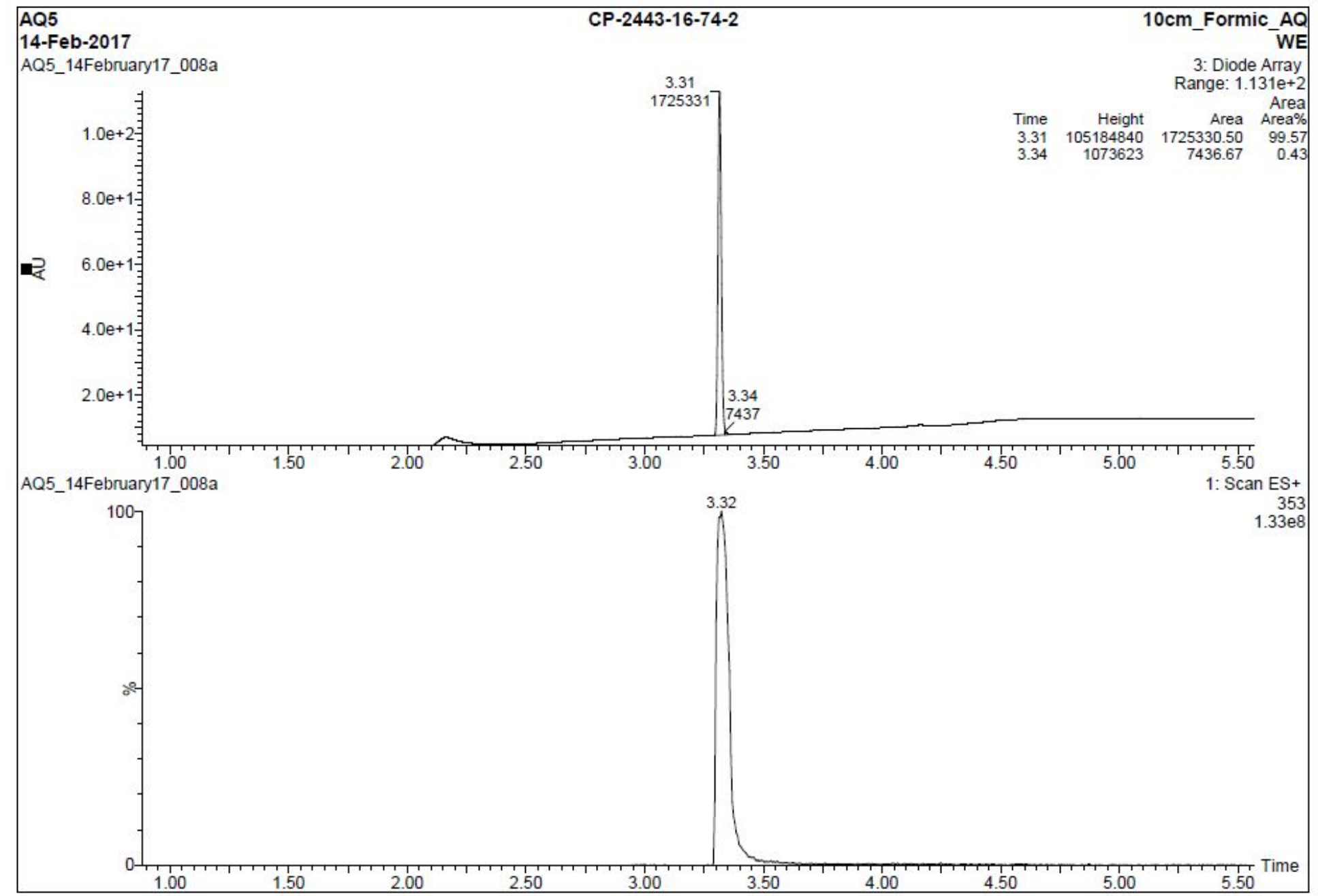


2-((1R,2S, 5S)-2-Benzyl-3-azabicyclo[3.1.0]hexan-3-yl)-6-((R)-2-methylmorpholino)pyrimidin-4(3H)-one (65)

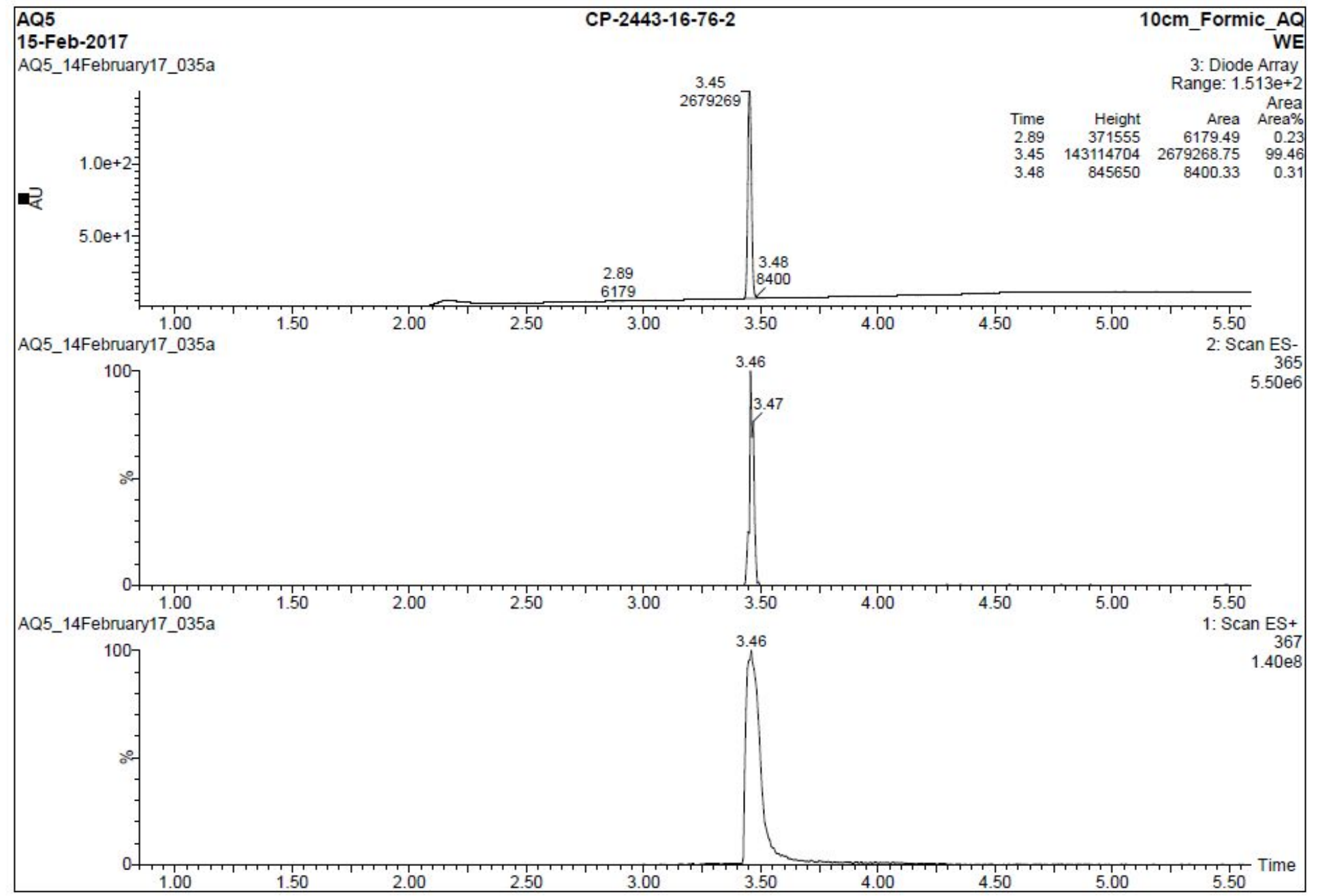


2-((1S,2S,5R)-2-Benzyl-3-azabicyclo[3.1.0]hexan-3-yl)-6-morpholinopyrimidin-4(3H)-one (66)

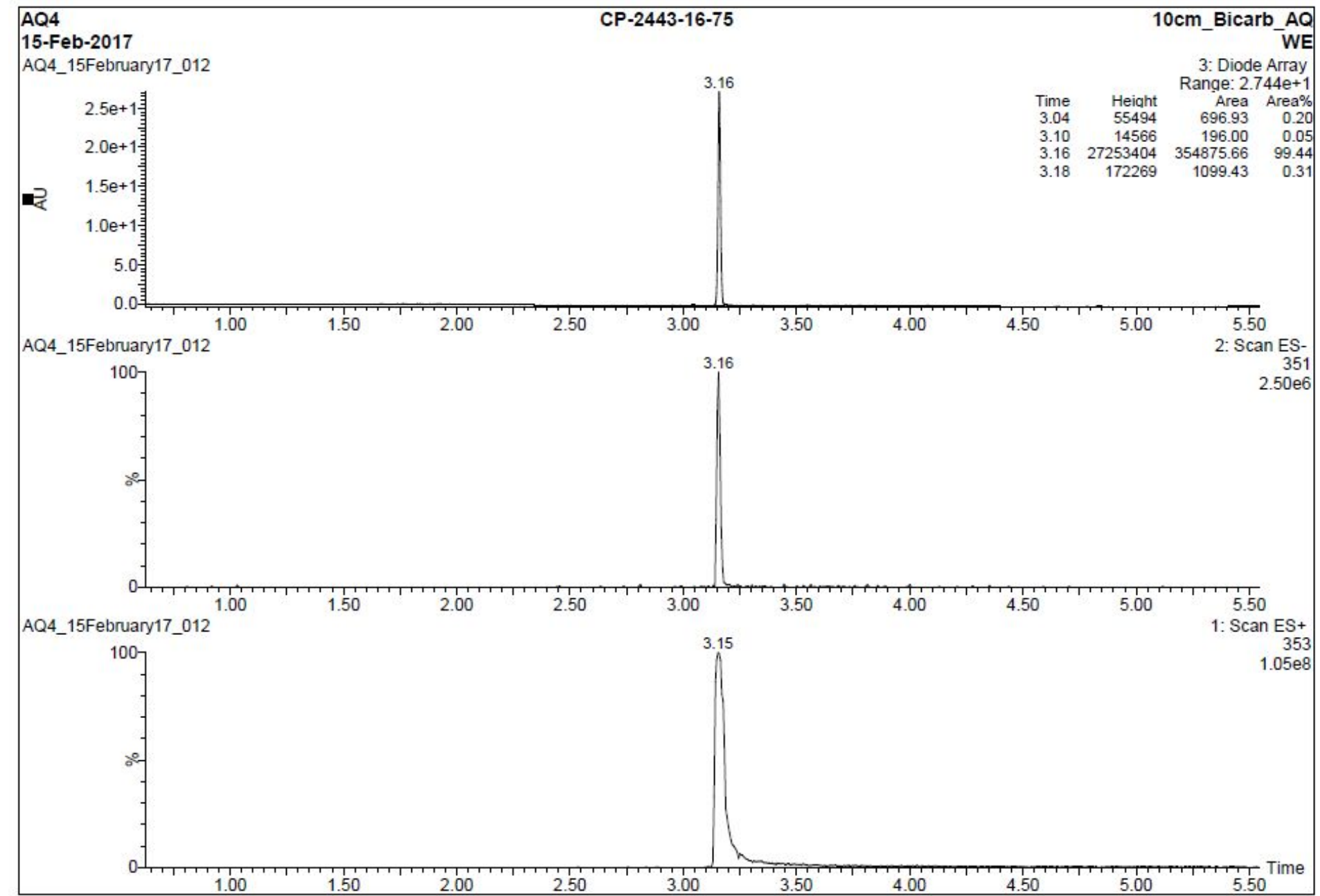


2-((1S,2S,5R)-2-Benzyl-3-azabicyclo[3.1.0]hexan-3-yl)-6-((R)-2-methylmorpholino)pyrimidin-4(3H)-one (67)

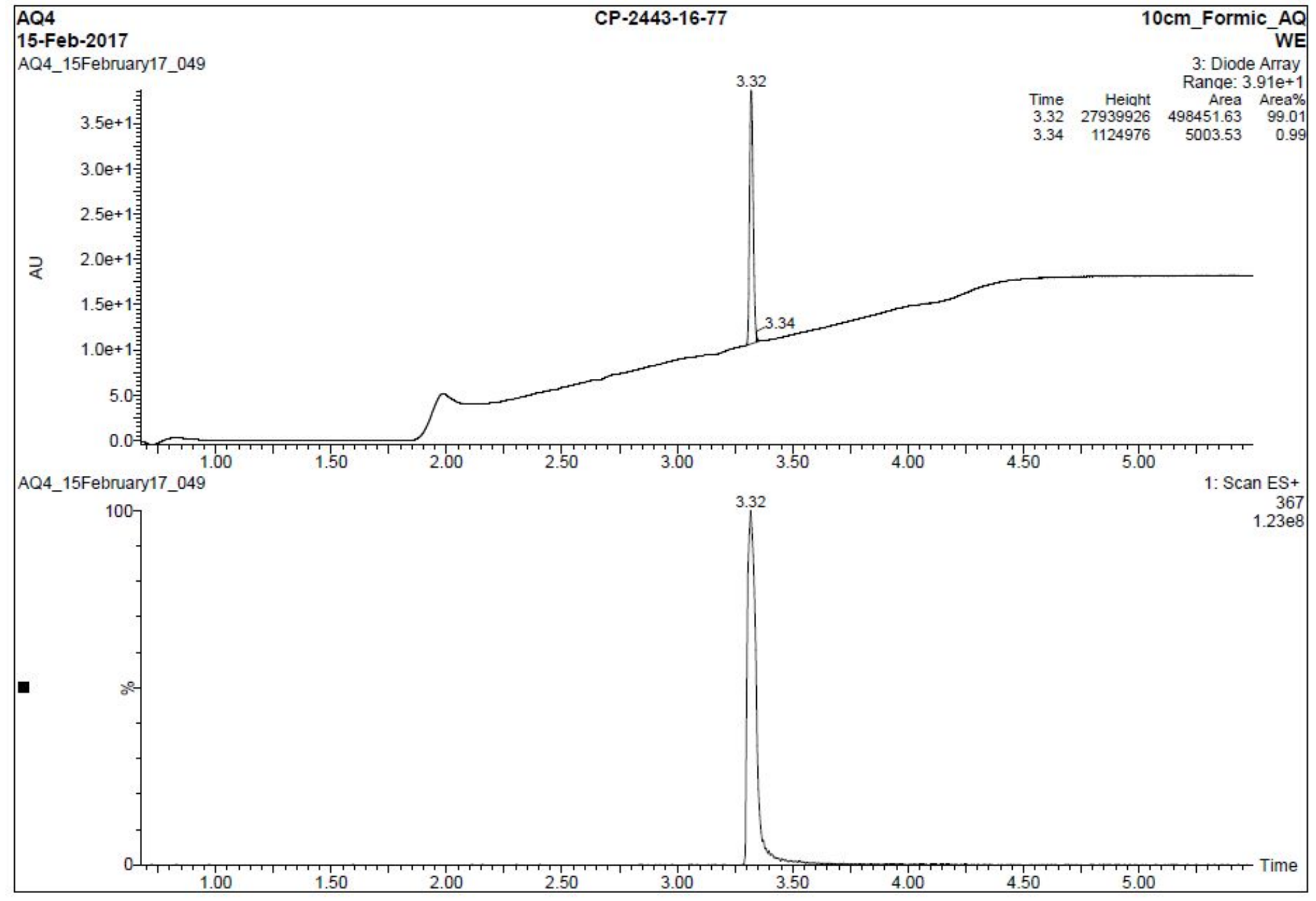


2-((2S,3R)-2-Benzyl-3-methylpyrrolidin-1-yl)-6-morpholinopyrimidin-4(3H)-one (68)

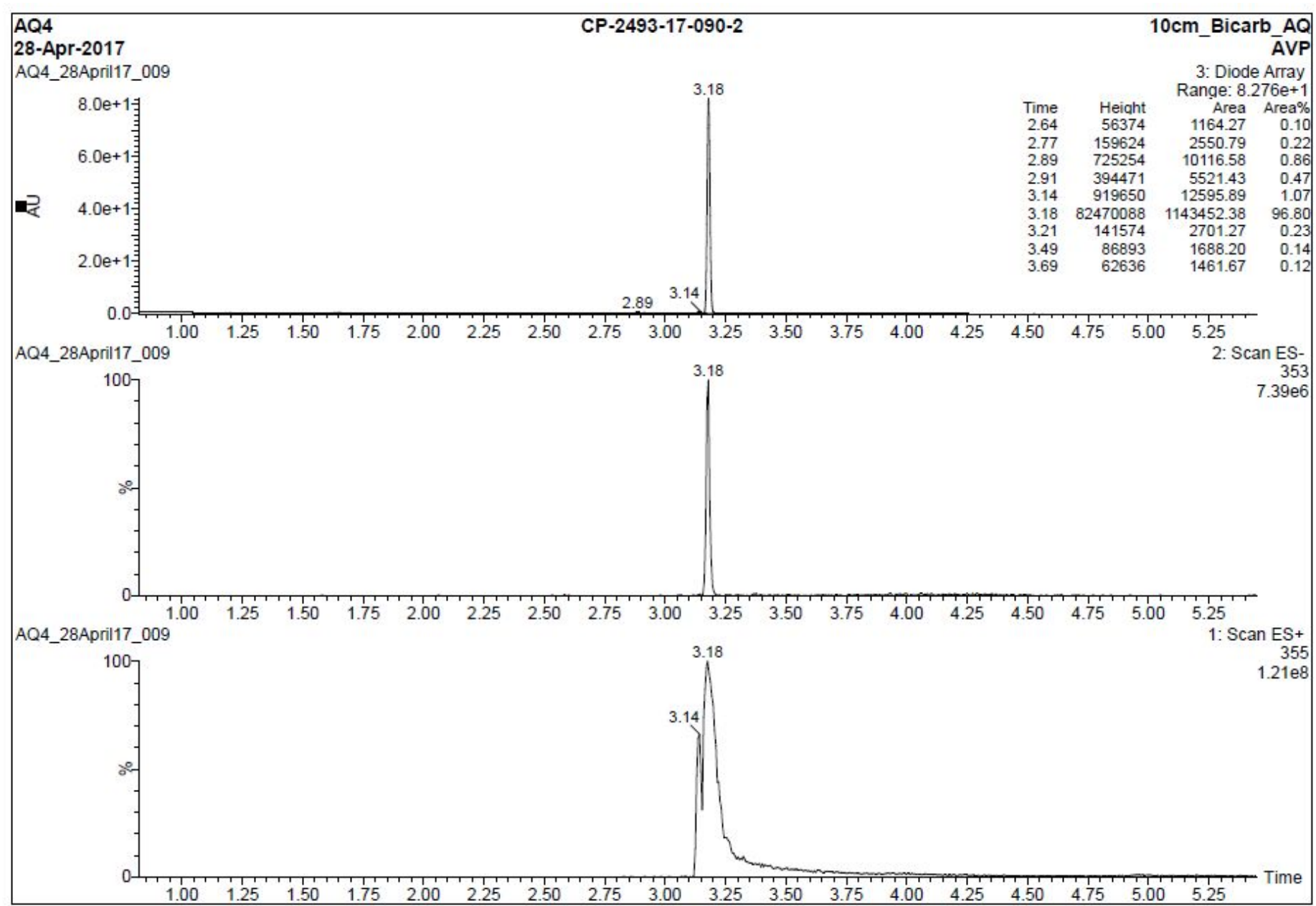


Biological data: Statistical analysis

Activities are reported in micromolar. The data for each of the compounds in Table S1 below represents the geometric mean of 2-7 independent tests, and the limits of the $95 \%$ confidence interval are given. Values marked greater than $(>)$ showed less than $50 \%$ inhibition at the highest concentration tested in at least one run.

Table S1: Biological activity and statistical analysis for the synthesised ATM inhibitors

\begin{tabular}{|c|c|c|c|c|c|}
\hline Compound & $\mathrm{ATM} \mathrm{K}_{\mathrm{i}}$ & ATM cell $\mathrm{IC}_{50}$ & ATR cell $\mathrm{IC}_{50}$ & $\mathrm{Vps} 34 \mathrm{~K}_{\mathrm{i}}$ & DNA-PK K ${ }_{i}$ \\
\hline 4 & $0.0005 \pm 0.0002$ & $1.3 \pm 0.3$ & $>30$ & $0.0145 \pm 0.01$ & $0.14 \pm 0.19$ \\
\hline 7 & $0.004 \pm 0.0016$ & $0.88 \pm 0.47$ & $>30$ & $0.126 \pm 0.100$ & $0.11 \pm 0.097$ \\
\hline 8 & $0.026 \pm 0.015$ & $>26$ & $>30$ & $>5$ & $0.016 \pm 0.011$ \\
\hline 11 & $>1.2$ & $>30$ & & $0.910 \pm 0.079$ & \\
\hline 16 & $0.10 \pm 0.10$ & $>30$ & $>30$ & & \\
\hline 19 & $>1$ & $>30$ & & & \\
\hline 25 & $>1.6$ & $>30$ & & $>5$ & \\
\hline 26 & $0.025 \pm 0.016$ & $6.7 \pm 4.5$ & $>30$ & $0.035 \pm 0.006$ & \\
\hline 30 & $0.087 \pm 0.099$ & $>30$ & & $0.27 \pm 0.31$ & \\
\hline 31 & $0.0012 \pm 0.001$ & $0.45 \pm 0.48$ & $>20$ & $0.016 \pm 0.018$ & $0.056 \pm 0.058$ \\
\hline 32 & $0.0001 \pm 0.0001$ & $0.2 \pm 0.12$ & $3 \pm 2$ & $0.0079 \pm 0.0058$ & $0.46 \pm 0.43$ \\
\hline 34 & $0.16 \pm 0.073$ & $>30$ & $>30$ & $3.3 \pm 2.7$ & \\
\hline 40 & $>1.6$ & $>30$ & & $>5$ & \\
\hline 43 & $0.030 \pm 0.0085$ & $4.0 \pm 2.5$ & $>25$ & $0.049 \pm 0.0093$ & \\
\hline 44 & $0.0056 \pm 0.0048$ & $3.4 \pm 4.4$ & $>30$ & $0.0092 \pm 0.0052$ & $0.089 \pm 0.023$ \\
\hline 45 & $>0.9$ & $>30$ & $>29$ & $0.63 \pm 0.49$ & \\
\hline
\end{tabular}




\begin{tabular}{|c|c|c|c|c|c|}
\hline $\mathbf{4 6}$ & $>1.2$ & $>30$ & $>30$ & & \\
\hline $\mathbf{4 7}$ & $0.006 \pm 0.0027$ & $0.92 \pm 0.008$ & $>30$ & $0.039 \pm 0.0053$ & $0.047 \pm 0.016$ \\
\hline $\mathbf{4 8}$ & $0.0027 \pm 0.0029$ & $1.3 \pm 1.1$ & $>30$ & $0.11 \pm 0.14$ & $0.025 \pm 0.05$ \\
\hline $\mathbf{4 9}$ & $0.023 \pm 0.012$ & $3.5 \pm 1.5$ & & & $0.039 \pm 0.042$ \\
\hline $\mathbf{5 0}$ & $0.0003 \pm 0.0001$ & $0.15 \pm 0.13$ & $6.5 \pm 4.9$ & $0.0035 \pm 0.0025$ & $0.0072 \pm 0.0038$ \\
\hline $\mathbf{5 1}$ & $0.0041 \pm 0.0041$ & $0.52 \pm 0.25$ & $12.6 \pm 6.4$ & $0.75 \pm 0.79$ & $0.16 \pm 0.14$ \\
\hline $\mathbf{5 2}$ & $0.0016 \pm 0.0012$ & $0.29 \pm 0.26$ & $>30$ & $0.098 \pm 0.078$ & $0.069 \pm 0.041$ \\
\hline $\mathbf{5 3}$ & $0.011 \pm 0.0025$ & $1.04 \pm 0.032$ & $>24$ & $>5$ & $0.44 \pm 0.35$ \\
\hline $\mathbf{5 4}$ & $0.0083 \pm 0.0056$ & $2.48 \pm 2.03$ & $>30$ & $0.17 \pm 0.13$ & $0.18 \pm 0.13$ \\
\hline $\mathbf{5 5}$ & $0.0015 \pm 0.0013$ & $0.23 \pm 0.055$ & $>30$ & $0.05 \pm 0.034$ & $0.37 \pm 0.21$ \\
\hline $\mathbf{5 6}$ & $0.0086 \pm 0.0007$ & $1.6 \pm 1.0$ & $>25$ & $1.8 \pm 2.6$ & $0.46 \pm 0.43$ \\
\hline $\mathbf{6 2}$ & $0.0001 \pm 0.0001$ & $0.2 \pm 0.115$ & $3 \pm 2$ & $0.0079 \pm 0.0058$ & $0.063 \pm 0.091$ \\
\hline $\mathbf{6 3}$ & $0.0011 \pm 0.0001$ & $0.82 \pm 0.19$ & $5.8 \pm 1.4$ & $0.84 \pm 0.40$ & $0.040 \pm 0.0066$ \\
\hline $\mathbf{6 4}$ & $0.0002 \pm 0.0001$ & $0.13 \pm 0.061$ & $10.5 \pm 2$ & $0.021 \pm 0.019$ & 0.040 \\
\hline $\mathbf{6 5}$ & $0.0017 \pm 0.0002$ & $0.56 \pm 0.29$ & $8.9 \pm 8.9$ & $0.88 \pm 0.72$ & $0.0058 \pm 0.0008$ \\
\hline $\mathbf{6 6}$ & $0.0006 \pm 0.0005$ & $0.45 \pm 0.39$ & $3.9 \pm 2.1$ & $0.0054 \pm 0.0048$ & $0.061 \pm 0.064$ \\
\hline $\mathbf{6 7}$ & $0.0009 \pm 0.0009$ & $1.31 \pm 1.09$ & $7.9 \pm 6.4$ & $1.5 \pm 1.7$ & $0.002 \pm 0.0018$ \\
\hline $\mathbf{6 8}$ & $0.0004 \pm 0.0011$ & $0.09 \pm 0.042$ & $5.5 \pm 6.9$ & $0.0064 \pm 0.0066$ & $0.11 \pm 0.086$ \\
\hline
\end{tabular}


Table S2: Mouse PK parameters for compound 55 after dosing at $2 \mathrm{mg} / \mathrm{kg} \mathrm{IV,} 10 \mathrm{mg} / \mathrm{kg}$ $P O$ and $30 \mathrm{mg} / \mathrm{kg}$ PO

\begin{tabular}{|c|c|c|c|c|c|c|}
\hline \multirow[b]{2}{*}{ PK PARAMETER } & \multirow[b]{2}{*}{ UNITS } & \multicolumn{3}{|c|}{ Plasma } & \multicolumn{2}{|c|}{ Brain } \\
\hline & & $\begin{array}{l}\text { IV } \\
2 \mathrm{mg} / \mathrm{kg} \\
\end{array}$ & $\begin{array}{l}P O \\
10 \mathrm{mg} / \mathrm{kg} \\
\end{array}$ & $\begin{array}{l}\mathrm{PO} \\
30 \mathrm{mg} / \mathrm{kg}\end{array}$ & $\begin{array}{l}P O \\
10 \mathrm{mg} / \mathrm{kg} \\
\end{array}$ & $\begin{array}{l}\mathrm{PO} \\
30 \mathrm{mg} / \mathrm{kg}\end{array}$ \\
\hline AUC(0 last) & $\mathrm{nM} \mathrm{h}$ & 2600 & 8600 & 44000 & 3900 & 20000 \\
\hline$A U C(0$-inf) & $\mathrm{nM} \mathrm{h}$ & 2600 & 8800 & 46000 & 4000 & 20000 \\
\hline AUCnorm & $\mathrm{nM} \mathrm{h} \mathrm{kg/mg}$ & 1300 & 880 & 1500 & 400 & 680 \\
\hline Oral Bioavailability (F) & $\%$ & NA & 68 & 115 & NA & NA \\
\hline Observed $\mathrm{C}_{\max }$ & $\mathrm{nM}$ & NA & 2900 & 17000 & 2000 & 9100 \\
\hline Observed $\mathrm{C}_{\max }$ Norm & $\mathrm{nM} \mathrm{kg} / \mathrm{mg}$ & NA & 290 & 560 & 200 & 300 \\
\hline Observed $\mathrm{T}_{\max }$ & $\mathrm{h}$ & NA & 0.5 & 0.25 & 0.5 & 0.5 \\
\hline Clearance (CL) & $\mathrm{L} / \mathrm{h} / \mathrm{kg}$ & 2.1 & NA & NA & NA & NA \\
\hline $\begin{array}{l}\begin{array}{l}\text { Volume of Distribution at Steady State } \\
\text { (Vdss) }\end{array} \\
\end{array}$ & $\mathrm{L} / \mathrm{kg}$ & 3.4 & NA & NA & NA & NA \\
\hline Mean Residency Time (MRT) & h & 1.6 & NA & NA & NA & NA \\
\hline Half-life (t $\left.t^{1} / 2\right)$ & $\mathrm{h}$ & 1.9 & 2.4 & 3.1 & 2.2 & 2.6 \\
\hline Half-life Regression Time Points & h & $1,2,4,8,12$ & $1,2,4,8,12$ & $1,2,4,8,12$ & $1,2,4,8,12$ & $1,2,4,8,12$ \\
\hline
\end{tabular}

\section{Vps34 ATM surrogate crystal structures}

\section{Purification of Vps34 ATM surrogate protein}

In brief, Vps34, comprising residues 268-879 was cloned into an E. coli expression vector, adding a TEV cleavable 6 His tag at its $\mathrm{N}$-terminus. The following mutations were introduced by site-directed mutagenesis: F612A, L616N, I634L, M682L, F684W, along with a glycine residue insertion between Gln686 and Ser687. The mutant construct was expressed in E. coli BL21 (DE3) cells. The protein was purified by immobilized metal affinity chromatography (IMAC) followed by His tag removal and size exclusion chromatography. The protein was eluted in $25 \mathrm{mM}$ Tris $\mathrm{pH} 8.0,250 \mathrm{mM} \mathrm{NaCl}$ and $0.5 \mathrm{mM}$ THP. The protein was concentrated to $8.5 \mathrm{mg} / \mathrm{mL}$ prior to co-crystallization.

\section{Crystallization, structure determination and analysis}

A $20 \mathrm{mM}$ stock solution of compound 44 in 100\% v/v DMSO was added to the purified Vps34 ATM surrogate protein to a final ligand concentration of $1 \mathrm{mM}$, and the complex was incubated 
at $\mathrm{r} . \mathrm{t}$ for $1 \mathrm{~h}$ before crystallization trials were set up. Co-crystals were grown using the hanging drop method of vapour diffusion, with streak seeding, over reservoirs containing 1.4 M sodium malonate $\mathrm{pH}$ 7.0, 0.1 M BIS-TRIS propane $\mathrm{pH}$ 7.0. Co-crystals were cryo-cooled for data collection by briefly transferring them to a drop containing precipitant solution plus $20 \% \mathrm{v} / \mathrm{v}$ glycerol, before plunging them into liquid nitrogen.

Data sets were collected at beamline id30a-1 at the ESRF synchrotron in Grenoble, France. The diffraction data were indexed, integrated and scaled using MOSFLM and AIMLESS. Details of data collection and processing are given in Table S3 below.

The Vps34 coordinates (PDB ID: 3LS8) were used as a search model for molecular replacement (MR) with the compound $\mathbf{4 4}$ data. In this search model, side-chains were truncated at $\mathrm{C} \beta$ for non-identical amino acids. MR was carried out in PHASER, locating one protein chain per asymmetric unit.

The difference electron density maps calculated after MR and initial refinement in REFMAC5 were used to check, and if necessary, re-model the protein conformation manually in COOT. Any missing side-chain atoms that were supported by the electron density maps were added to the model. The hinge was also re-modelled to accommodate the inserted glycine residue. After another round of refinement in REFMAC5, the maps were further examined for the presence of compound. Once the ligand electron density was located, a molecular structure file and refinement library file were produced using ACEDRG. The compound was fitted into the electron density maps using COOT and refined using REFMAC5. Water molecules were then added using the water placement option in COOT and refined using REFMAC5. Details of structure refinement and final model analysis are given in Table S3 below. 
Table S3: Details of data collection, processing and structure refinement statistics for the VPS34 ATM surrogate protein structure in complex with compound 44.

\begin{tabular}{|c|c|}
\hline Compound No. & 44 \\
\hline \multicolumn{2}{|c|}{ Data collection and processing statistics } \\
\hline Unit cell dimensions $(\AA)$ & $\begin{array}{c}a=79.86 \\
b=168.12 \\
c=61.69\end{array}$ \\
\hline Space group & $\mathrm{P} 21212$ \\
\hline Resolution range $(\AA)$ & $\begin{array}{c}49.73-3.12(3.34- \\
3.12) \\
\end{array}$ \\
\hline No. of unique reflections & $15383(2732)$ \\
\hline Completeness (\%) & $99.8(99.9)$ \\
\hline $\mathrm{CC}(1 / 2)$ & $0.999(0.500)$ \\
\hline \multicolumn{2}{|c|}{ Structure refinement statistics } \\
\hline Resolution range $(\AA)$ & $84.06-3.12$ \\
\hline No. of reflections (working/test) & $14568 / 815$ \\
\hline $\mathbf{R}_{\text {work }} / \mathbf{R}_{\text {free }}(\%)$ & $21.3 / 26.2$ \\
\hline Rmsd bond lengths $(\AA) /$ angles $\left(^{\circ}\right)$ & $0.011 / 1.329$ \\
\hline No. of protein atoms & 4442 \\
\hline No. of solvent atoms & 1 \\
\hline No. of ligand atoms & 25 \\
\hline Mean overall B value $\left(\AA^{2}\right)$ & 62.4 \\
\hline Mean water B value $\left(\AA^{2}\right)$ & 30.2 \\
\hline Mean ligand $B$ value $\left(\AA^{2}\right)$ & 44.7 \\
\hline $\begin{array}{l}\text { Ramachandran plot favoured } \\
\text { region }\end{array}$ & $532(96.2 \%)$ \\
\hline Ramachandran plot allowed region & $20(3.6 \%)$ \\
\hline Ramachandran plot outlier region & $1(0.2 \%)$ \\
\hline
\end{tabular}

